Aproximação na esfera por uma soma com pesos de harmônicos esféricos

\author{
Ana Carla Piantella
}





\title{
Aproximação na esfera por uma soma com pesos de harmônicos esféricos ${ }^{1}$
}

\author{
Ana Carla Piantella
}

Orientador: Prof. Dr. Valdir Antonio Menegatto

Tese apresentada ao Instituto de Ciências Matemáticas e de Computação da Universidade de São Paulo, como parte dos requisitos para obtenção do título de Doutor em Ciências: Matemática.

USP - São Carlos

Janeiro/2007

\footnotetext{
${ }^{1}$ Este trabalho teve suporte financeiro da CAPES
} 

Aos meus pais,

José Antonio e Sonia, com carinho... 



\section{Agradecimentos}

A Deus, por sempre me iluminar nos momentos mais difíceis, por ter me dado forças e me mostrado o caminho para conseguir chegar ao final de mais uma etapa da minha vida.

Ao meu orientador, Prof. Valdir Antonio Menegatto, pela tão dedicada orientação e por não ter medido esforços para me ajudar sempre que precisei. Agradeço pela sua disposição, paciência, amizade, incentivo e pelo exemplo de profissional que mostrou ser durante todos esses anos.

Aos meus pais, que com seus ensinamentos e carinho sempre me incentivaram a buscar os meus sonhos, mesmo que isso me levasse a ficar um pouquinho distante deles. Muito obrigada por todo apoio e amor que sempre demonstraram, e por tudo que fizeram para tentar me dar o melhor. Amo vocês!

A toda minha família, que sempre torceu por mim e sempre demonstrou muito carinho.

Aos professores Ana Paula Peron e Claudemir Pinheiro de Oliveira, pela ajuda no trabalho, pelas sugestões e pela amizade. Agradeço a todos os professores que participaram da minha vida acadêmica, em especial, Luiz Alberto Duran Salomão, Walter dos Santos Motta Jr. e Geraldo M. de Azevedo Botelho pela orientação no período da graduação.

Aos meus amigos, pela amizade e pelo carinho. Agradeço em especial, minhas "irmãs de república": Chris e Karen, e os meus amigos do ICMC: Grazielle, Judith, Mário, Michelle, Ronaldinho e Thiago, pela ajuda e pelos bons momentos que passamos juntos, e que com certeza tornaram os dias em São Carlos mais alegres e mais agradáveis.

Aos funcionários do ICMC por serem tão prestativos e atenciosos, e a todos os que colaboraram para a realização deste trabalho.

À CAPES pelo suporte financeiro. 



\section{Abstract}

The subject of this work is to study approximation on the sphere by weighted sums of spherical harmonics. We present necessary and sufficient conditions on the weights for convergence in both, the continuous and the $L^{p}$ cases. We analyse the convergence rates of the approximation processes using a modulus of smoothness related to the strong LaplaceBeltrami derivative. We include proofs for several results related to such a derivative, since we were unable to find them in the literature. 



\section{Resumo}

O objetivo deste trabalho é estudar aproximação na esfera por uma soma com pesos de harmônicos esféricos. Apresentamos condições necessárias e suficientes sobre os pesos para garantir a convergência, tanto no caso contínuo quanto no caso $L^{p}$. Analisamos a ordem de convergência dos processos aproximatórios usando um módulo de suavidade esférico relacionado à derivada forte de Laplace-Beltrami. Incluímos provas para vários resultados sobre a derivada forte de Laplace-Beltrami, já que não conseguimos encontrá-las na literatura. 



\section{Sumário}

$\begin{array}{ll}\text { Introdução } & 1\end{array}$

1 Preliminares $\quad 5$

1.1 Os harmônicos esféricos . . . . . . . . . . . . . . . . . . . 5

1.2 O Teorema da Adição e a Fórmula de Funk-Hecke . . . . . . . . . . . . . . . . 11

1.3 Fórmula de linearização de Dougall . . . . . . . . . . . . . . . . . . . . . . . 13

2 A derivada forte de Laplace-Beltrami e operadores associados $\quad 17$

2.1 A projeção esférica . . . . . . . . . . . . . . . . . . 17

2.2 O operador translação esférica . . . . . . . . . . . . . . . . . . . . . . 21

2.3 A derivada forte de Laplace-Beltrami . . . . . . . . . . . . . . . . . . . . . 27

2.4 A integral de Laplace-Beltrami . . . . . . . . . . . . . . . . . . . . 31

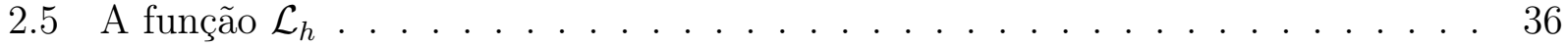

2.6 O operador $A_{h}^{r} \ldots \ldots \ldots \ldots \ldots \ldots \ldots$

2.7 Uma caracterização para $W_{X}^{r} \quad \ldots \ldots \ldots \ldots \ldots$

2.8 O módulo de suavidade esférico e o K-funcional . . . . . . . . . . . . . . 51

2.9 Equivalência entre o módulo de suavidade e o $K$-funcional . . . . . . . . . . . 56

3 Aproximação por somas com pesos de harmônicos esféricos 59

3.1 O operador $T_{n} \ldots \ldots \ldots \ldots \ldots \ldots \ldots \ldots$

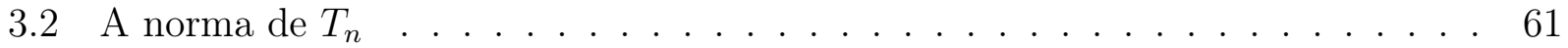

3.3 Aproximações da identidade . . . . . . . . . . . . . . . . . . . . 64

3.4 Um exemplo . . . . . . . . . . . . . . . . . . . . . . . . . . 71

3.5 Aproximação da identidade: o caso pontual . . . . . . . . . . . . . . . . 75

4 Ordem de convergência do operador $T_{n} \quad 83$

4.1 A ordem de aproximação . . . . . . . . . . . . . . . . . . . . . . . 83

$\begin{array}{ll}\text { Problemas abertos } & 91\end{array}$

$\begin{array}{ll}\text { Referências Bibliográficas } & 93\end{array}$ 
Tabela de símbolos

Índice Remissivo 


\section{Introdução}

A intersecção da Teoria da Aproximação com a Análise na Esfera é um tópico com aplicações em diversas áreas, dentre elas, a Geofísica, Geologia, Meteorologia, Oceanografia, Tomografia, Teoria do Potencial, etc ([27]). Nos casos mais importantes, as aplicações envolvem problemas práticos onde a esfera tri-dimensional serve como modelo para a superfície terrestre.

Um procedimento padrão para aproximar uma função $f$ em um espaço com produto interno, é considerar a série de Fourier da função em relação a um sistema ortogonal do espaço, e usar a seqüência das somas parciais da série como seqüência aproximadora. Esse procedimento é clássico dentro da Teoria da Aproximação como pode-se comprovar em muitas referências na literatura, por exemplo, ([32]), ([7], Cap. VIII).

No caso em que $S$ é um intervalo fechado, existe uma função contínua com domínio $S$ para a qual a série de Fourier correspondente não converge para $f$ em relação a norma uniforme ([14]). Dessa forma, neste e em outros casos, a solução alternativa para o processo de aproximação é considerar expansões com pesos e estudar a convergência baseada na escolha dos pesos. Surgem, então, algumas perguntas:

(i) Como escolher os pesos para garantir a convergência para toda função do espaço ambiente; (ii) Garantida a convergência, estudar o comportamento das ordens de convergência;

(iii) Como escolher os pesos de forma que os operadores de aproximação dados pela série de Fourier truncada tenham propriedades semelhantes a de outros operadores conhecidos.

Vários artigos recentes de R. Lasser e J. Obermaier (veja por exemplo, [15] e [16]) discutiram problemas semelhantes aos acima descritos, bem como outros relacionados. Precisamente, os artigos tratam de expansões de Fourier com pesos, em relação a sistemas de polinômios ortogonais, considerando o espaço usual $C(S)$ de funções contínuas e os espaços $L^{p}(S, \pi)$, $1 \leq p<\infty$, onde $S \subset \mathbb{R}$ é o suporte compacto de uma medida de probabilidade $\pi$.

Neste trabalho consideraremos os problemas citados nos itens (i)-(iii) acima para funções definidas em $S^{m}$, a esfera unitária em $\mathbb{R}^{m+1}, m \geq 2$.

No primeiro passo, estudaremos a aproximação de uma tal função por somas com pesos de harmônicos esféricos. Procuraremos condições necessárias e suficientes sobre os pesos para garantir a convergência tanto no caso contínuo quanto no caso $L^{p}$. 
No segundo passo, tentaremos analisar a razão de convergência. Dentre os módulos de suavidade disponíveis na literatura, optamos pela análise da razão de convergência usando o módulo de suavidade esférico introduzido por M. Wehrens. Tal módulo já foi discutido em algumas situações por P. I. Lizorkin, S. Pawelke e P. L. Butzer ([18, 26, 36, 37]). A análise das propriedades desse módulo de suavidade passa naturalmente pelo estudo da derivada forte de Laplace-Beltrami e do operador translação esférica. Um tratamento formal para a derivada forte de Laplace-Beltrami no caso tridimensional foi dado por Wehrens em sua tese de doutorado ([37]), enquanto que uma descrição de como alguns resultados ficariam no caso geral foi incluída, sem provas, em [36]. O trabalho de Wehrens foi baseado em um artigo de W. Rudin ([28]), que considerou a versão pontual da derivada forte de Laplace-Beltrami para estabelecer sua teoria de unicidade para séries de Laplace. $\mathrm{Na}$ verdade, os resultados em [28] são bem semelhantes àqueles em [36], mas com um conjunto de hipóteses ligeiramente diferente. Dessa forma, faz parte de nosso trabalho ratificar os resultados listados em [36], incluindo provas detalhadas para todos os resultados lá mencionados bem como a discussão de outros que se fizeram necessários ao longo do trabalho. Apresentaremos também um estudo dos operadores associados à derivada forte de Laplace-Beltrami e ao módulo de suavidade esférico. Como conseqüência, apresentaremos uma descrição dos espaços de funções suaves no sentido da derivada forte de Laplace-Beltrami.

O trabalho propriamente dito será apresentado da seguinte forma.

No Capítulo 1, apresentaremos todo o material básico necessário no desenvolvimento do trabalho, ou seja discutiremos rapidamente os seguintes itens: harmônicos esféricos, polinômios de Legendre, fórmula da adição para harmônicos esféricos, teorema de Funk-Hecke, convolução esférica e fórmula de linearização de Dougall.

No Capítulo 2, introduziremos os operadores projeção esférica e translação esférica para em seguida apresentar a derivada forte de Laplace-Beltrami. Após estabelecer vários resultados envolvendo a derivada forte, introduziremos e analisaremos o operador integral de LaplaceBeltrami. Nas seções finais do capítulo, apresentaremos caracterizações para os espaços de funções suaves no sentido da derivada forte de Laplace-Beltrami e deduziremos algumas propriedades do módulo de suavidade esférico, dentre elas a equivalência deste com o $K$-funcional correspondente.

Finalmente, no Capítulo 3, estudaremos aproximações de uma função definida na esfera por somas com pesos de harmônicos esféricos. Investigaremos as propriedades básicas do operador de aproximação, calcularemos sua norma e consideraremos tanto aproximações globais quanto aproximações pontuais, válidas para todas as funções do espaço envolvido. Além disso, apresentaremos um exemplo concreto de construção de um operador de aproximação.

No Capítulo 4, utilizaremos os resultados do Capítulo 2, para analisar ordens de convergência do operador de aproximação em alguns casos específicos. 
Finalizaremos o trabalho apresentando alguns problemas abertos que colocamos como propostas de trabalho para o futuro. 


\section{Capítulo}

\section{Preliminares}

Neste capítulo, apresentaremos alguns resultados da análise em $S^{m}$. Quase todo esse material será utilizado na dedução dos resultados dos capítulos posteriores. Uma ampla discussão sobre esses resultados, incluindo provas e aplicações dos mesmos, podem ser encontrados em várias referências, dentre elas [2], [11], [22], [24], [23] e [31].

\subsection{Os harmônicos esféricos}

Os harmônicos esféricos desempenham em $S^{m}, m \geq 2$, o papel desempenhado pelas funções seno e cosseno no estudo de funções periódicas no círculo $S^{1}$. Precisamente, enquanto uma função definida em $S^{1}$ possui sua expansão em série de Fourier usual, funções definidas em $S^{m}, m \geq 2$, possuem uma expansão de Fourier em termos de harmônicos esféricos. Antes de explicarmos isso em detalhes, introduziremos algumas notações.

Seja $d \sigma_{m}$ o elemento de medida usual sobre $S^{m}$ normalizada por

$$
\sigma_{m}:=\int_{S^{m}} d \sigma_{m}=\frac{2 \pi^{(m+1) / 2}}{\Gamma((m+1) / 2)},
$$

onde $\Gamma$ denota a função gama usual.

Denotaremos por $C\left(S^{m}\right)$ o espaço das funções contínuas definidas em $S^{m}$, munido de sua norma uniforme

$$
\|f\|_{\infty}:=\sup _{x \in S^{m}}|f(x)|, \quad f \in C\left(S^{m}\right),
$$

e por $L^{p}\left(S^{m}\right), 1 \leq p<\infty$, o espaço das funções $f$ definidas em $S^{m}$ tal que $|f|^{p}$ é integrável em relação a $d \sigma_{m}$, onde identificamos funções que coincidem em $S^{m}$ exceto em um conjunto de medida nula. A norma nesses espaços é dada por

$$
\|f\|_{p}:=\left(\frac{1}{\sigma_{m}} \int_{S^{m}}|f(x)|^{p} d \sigma_{m}(x)\right)^{1 / p}, \quad f \in L^{p}\left(S^{m}\right) .
$$

A menos de especificação em contrário, $X$ denotará um dos espaços acima e $\|\cdot\|_{X}$ a norma correspondente. Observamos também que em todo o trabalho o espaço $\mathbb{R}^{m+1}$ será munido com a norma euclidiana. 
Usaremos a estrutura métrica do espaço $L^{2}\left(S^{m}\right)$, ou seja aquela determinada pelo produto interno

$$
\langle f, g\rangle_{2}=\frac{1}{\sigma_{m}} \int_{S^{m}} f(x) \overline{g(x)} d \sigma_{m}(x), \quad f, g \in L^{2}\left(S^{m}\right),
$$

No que segue, ortogonalidade sempre referir-se-à ao produto interno (1.1). Observamos ainda que neste trabalho $\mathbb{N}$ denotará o conjunto $\mathbb{N}:=\{0,1,2, \ldots\}$.

Dizemos que uma função $f: \mathbb{R}^{m+1} \rightarrow \mathbb{C}$ é homogênea de grau $n$ quando

$$
f(\lambda x)=\lambda^{n} f(x), \quad \lambda>0, \quad x \in \mathbb{R}^{m+1} .
$$

Ela é dita ser harmônica quando $\Delta(f)=0$, onde $\Delta=\sum_{j=1}^{m+1} \partial^{2} / \partial x_{j}^{2}$.

Denotaremos por $\mathcal{P}_{n}\left(\mathbb{R}^{m+1}\right)$ o espaço formado pelos polinômios em $m+1$ variáveis que são homogêneos de grau $n$ e por $\mathcal{H}_{n}\left(\mathbb{R}^{m+1}\right)$ o espaço dos polinômios em $m+1$ variáveis que são harmônicos e homogêneos de grau $n$.

Definição 1.1.1. Um polinômio esférico de grau $n$ em $m+1$ variáveis é a restrição a $S^{m}$ de um polinômio de grau $n$ em $m+1$ variáveis. Denotaremos por $\mathbb{P}_{n}\left(S^{m}\right)$ o espaço dos polinômios esféricos de grau no máximo $n$ em $m+1$ variáveis e escreveremos $\mathbb{P}\left(S^{m}\right):=\cup_{n=0}^{\infty} \mathbb{P}_{n}\left(S^{m}\right)$.

Definição 1.1.2. Um harmônico esférico de grau $n$ em $m+1$ variáveis é a restrição a $S^{m}$ de um polinômio harmônico e homogêneo de grau n em $m+1$ variáveis. O espaço dos harmônicos esféricos de grau n em $m+1$ variáveis será denotado por $\mathcal{H}_{n}\left(S^{m}\right)$.

Denotaremos por $\mathcal{P}_{n}\left(S^{m}\right)$ o espaço das restrições a $S^{m}$ dos polinômios homogêneos de grau $n$ em $m+1$ variáveis .

Os resultados a seguir mostrarão que é possível construir uma base ortonormal de $\mathbb{P}_{n}\left(S^{m}\right)$ inteiramente composta de harmônicos esféricos.

Teorema 1.1.3. Se $l \neq k$, então $\mathcal{H}_{l}\left(S^{m}\right)$ e $\mathcal{H}_{k}\left(S^{m}\right)$ são subespaços ortogonais de $L^{2}\left(S^{m}\right)$.

Demonstração: Sejam $p \in \mathcal{H}_{l}\left(\mathbb{R}^{m+1}\right)$ e $q \in \mathcal{H}_{k}\left(\mathbb{R}^{m+1}\right)$. Pela Identidade de Green temos que

$$
\int_{S^{m}}\left(p D_{\vec{n}}(q)-q D_{\vec{n}}(p)\right) d \sigma_{m}=\int_{B_{m}}(p \Delta(q)-q \Delta(p))(x) d x=0,
$$

onde $D_{\vec{n}}(p)$ e $D_{\vec{n}}(q)$ são, respectivamente, as derivadas direcionais de $p$ e $q$ na direção do vetor normal $\vec{n}$ exterior a $S^{m}$ e $B_{m}$ é a bola aberta unitária com centro na origem de $\mathbb{R}^{m+1}$. Mas,

$$
D_{\vec{n}}(p)(\xi)=\left.\frac{d}{d t} p(t \xi)\right|_{t=1}=\left.\frac{d}{d t}\left(t^{l} p(\xi)\right)\right|_{t=1}=l p(\xi), \quad \xi \in S^{m}
$$

e, da mesma forma, $D_{\vec{n}}(q)(\xi)=k q(\xi), \xi \in S^{m}$. Logo,

$$
0=\int_{S^{m}}\left(p D_{\vec{n}}(q)-q D_{\vec{n}}(p)\right) d \sigma_{m}=\int_{S^{m}}(k p q-l p q) d \sigma_{m}=(k-l) \int_{S^{m}} p q d \sigma_{m} .
$$


Como $l \neq k$, segue que $p$ e $q$ são ortogonais.

Usando multi-índices $\alpha=\left(\alpha_{1}, \alpha_{2}, \ldots, \alpha_{m+1}\right)$ podemos representar um polinômio $p(x)$ de $\mathcal{P}_{n}\left(\mathbb{R}^{m+1}\right)$ da seguinte forma $([22])$ :

$$
p(x)=\sum_{|\alpha|=n} a_{\alpha} x^{\alpha}, \quad a_{\alpha} \in \mathbb{C}, \quad x=\left(x_{1}, x_{2}, \ldots, x_{m+1}\right),
$$

onde $x^{\alpha}:=x_{1}^{\alpha_{1}} x_{2}^{\alpha_{2}} \ldots x_{m+1}^{\alpha_{m+1}}$ e $|\alpha|:=\alpha_{1}+\alpha_{2}+\cdots+\alpha_{m+1}$. O operador diferencial associado a $p$ é o operador $p(D)$ dado por

$$
p(D):=\sum_{|\alpha|=n} a_{\alpha} D^{\alpha}, \quad D^{\alpha}=\frac{\partial^{\alpha_{1}}}{\partial x_{1}^{\alpha_{1}}} \frac{\partial^{\alpha_{2}}}{\partial x_{2}^{\alpha_{2}}} \cdots \frac{\partial^{\alpha_{m+1}}}{\partial x_{m+1}^{\alpha_{m+1}}} .
$$

Podemos definir o seguinte produto interno em $\mathcal{P}_{n}\left(\mathbb{R}^{m+1}\right)$ :

$$
[p, q]_{n}:=p(D)(\bar{q}), \quad p, q \in \mathcal{P}_{n}\left(\mathbb{R}^{m+1}\right)
$$

Se $p$ tem a representação acima e $q(x)=\sum_{|\beta|=n} b_{\beta} x^{\beta}$, podemos usar a fórmula

$$
D^{\alpha}\left(x^{\beta}\right):= \begin{cases}0 & \text { se } \alpha \neq \beta \text { e }|\alpha|=|\beta| \\ \alpha ! & \text { se } \alpha=\beta\end{cases}
$$

para concluir que

$$
[p, q]_{n}:=p(D)(\bar{q})=\sum_{|\alpha|=n} \sum_{|\beta|=n} a_{\alpha} \overline{b_{\beta}} D^{\alpha}\left(x^{\beta}\right)=\sum_{|\alpha|=n} \alpha ! a_{\alpha} \overline{b_{\alpha}}
$$

No próximo teorema utilizaremos a notação $[s], s \in \mathbb{R}$, para representar o maior inteiro menor ou igual a $s$.

Teorema 1.1.4. Se p é um elemento de $\mathcal{P}_{n}\left(\mathbb{R}^{m+1}\right)$, então

$$
p(x)=p_{n}(x)+|x|^{2} p_{n-2}(x)+\cdots+|x|^{2 k} p_{n-2 k}(x), \quad x \in \mathbb{R}^{m+1}
$$

onde $k=[n / 2]$ e $p_{n-2 j} \in \mathcal{H}_{n-2 j}\left(\mathbb{R}^{m+1}\right), j=0,1, \ldots, k$. A representação acima é única.

Demonstração: Se $n=0$ ou $n=1$, então $\mathcal{P}_{n}\left(\mathbb{R}^{m+1}\right)=\mathcal{H}_{n}\left(\mathbb{R}^{m+1}\right)$ e o teorema é válido trivialmente. Nos casos restantes mostraremos que

$$
\mathcal{P}_{n}\left(\mathbb{R}^{m+1}\right)=\mathcal{H}_{n}\left(\mathbb{R}^{m+1}\right) \oplus|x|^{2} \mathcal{P}_{n-2}\left(\mathbb{R}^{m+1}\right)
$$

onde $|x|^{2} \mathcal{P}_{n-2}\left(\mathbb{R}^{m+1}\right)=\left\{|x|^{2} q(x): q \in \mathcal{P}_{n-2}\left(\mathbb{R}^{m+1}\right)\right\}$ e a soma direta é ortogonal em relação ao produto interno $[\cdot, \cdot]_{n}$. Para tanto, vamos mostrar que o complemento ortogonal de $\mathcal{H}_{n}\left(\mathbb{R}^{m+1}\right)$ em $\mathcal{P}_{n}\left(\mathbb{R}^{m+1}\right)$ é $|x|^{2} \mathcal{P}_{n-2}\left(\mathbb{R}^{m+1}\right)$. Se $p \in \mathcal{P}_{n}\left(\mathbb{R}^{m+1}\right)$ é ortogonal a $|x|^{2} \mathcal{P}_{n-2}\left(\mathbb{R}^{r}\right)$, então

$$
0=\left[|x|^{2} q, p\right]_{n}=\Delta(q)(D)(\bar{p})=q(D)(\overline{\Delta(p)})=[q, \Delta(p)]_{n-2}, \quad q \in \mathcal{P}_{n-2}\left(\mathbb{R}^{m+1}\right)
$$


onde a última igualdade segue do fato de que $\Delta(p) \in \mathcal{P}_{n-2}\left(\mathbb{R}^{m+1}\right)$. Segue que $\Delta(p)$ é ortogonal a todo $q \in \mathcal{P}_{n-2}\left(\mathbb{R}^{m+1}\right)$, ou seja, $\Delta(p)=0$. Portanto, $p \in \mathcal{H}_{n}\left(\mathbb{R}^{m+1}\right)$. Consequentemente, $\left(|x|^{2} \mathcal{P}_{n-2}\left(\mathbb{R}^{m+1}\right)\right)^{\perp} \subset \mathcal{H}_{n}\left(\mathbb{R}^{m+1}\right)$. O procedimento acima é reversível, isto é, $\left(|x|^{2} \mathcal{P}_{n-2}\left(\mathbb{R}^{m+1}\right)\right)^{\perp} \supset \mathcal{H}_{n}\left(\mathbb{R}^{m+1}\right)$. A conclusão do teorema segue por indução.

Como todo polinômio é soma de polinômios homogêneos, o corolário abaixo segue imediatamente do teorema acima.

Corolário 1.1.5. Se p é um polinômio de grau n em $m+1$ variáveis, então a restrição de $p$ a $S^{m}$ é soma de harmônicos esféricos de grau no máximo n.

Na verdade, os resultados acima revelam que

$$
\mathbb{P}_{n}\left(S^{m}\right)=\mathcal{H}_{0}\left(S^{m}\right) \oplus \mathcal{H}_{1}\left(S^{m}\right) \oplus \cdots \oplus \mathcal{H}_{n}\left(S^{m}\right)
$$

O espaço $\mathcal{H}_{n}\left(S^{m}\right)$ tem dimensão $N(m, n)([1$, p. 82]) onde $N(m, 0)=1$ e

$$
N(m, n)=\left(\begin{array}{c}
m+n \\
m
\end{array}\right)-\left(\begin{array}{c}
m+n-2 \\
m
\end{array}\right)=\frac{2 n+m-1}{n}\left(\begin{array}{c}
n+m-2 \\
n-1
\end{array}\right), \quad n \geq 1 .
$$

Lembramos que se $\left\{x_{n}\right\}$ e $\left\{y_{n}\right\}$ são seqüências de números reais, a igualdade

$$
x_{n}=O\left(y_{n}\right) \quad(n \rightarrow \infty)
$$

é equivalente ao fato da seqüência $\left\{x_{n} / y_{n}\right\}$ ser limitada.

O próximo resultado pode ser encontrado em ([22], p. 17).

Proposição 1.1.6. Vale a seguinte igualdade:

$$
N(m, n)=O\left(n^{m-1}\right) \quad(n \rightarrow \infty) .
$$

Segue de (1.2) que a dimensão $d_{n}$ do espaço $\mathbb{P}_{n}\left(S^{m}\right)$ é

$$
d_{n}=\sum_{k=0}^{n} N(m, k) .
$$

Usando propriedades do fatorial podemos mostrar que

$$
d_{n}=\left(\begin{array}{c}
m+n \\
m
\end{array}\right)+\left(\begin{array}{c}
m+n-1 \\
m
\end{array}\right)
$$

Note ainda que

$$
d_{n} \geq \frac{2 n^{m}}{m !} .
$$

Proposição 1.1.7. Com as notações acima, vale a seguinte igualdade:

$$
\lim _{n \rightarrow \infty} \frac{N(m, n)}{d_{n}}=0
$$


Demonstração: Da Proposição 1.1.6 segue que existe uma constante positiva $C$ tal que

$$
N(m, n) \leq C n^{m-1}, \quad n \in \mathbb{N}
$$

Portanto, usando a Equação (1.6) vem que

$$
\frac{N(m, n)}{d_{n}} \leq \frac{C m !}{n}
$$

Tomando-se o limite quando $n \rightarrow \infty$ em ambos os lados da desigualdade acima obtemos

$$
\lim _{n \rightarrow \infty} \frac{N(m, n)}{d_{n}}=0
$$

completando a prova da proposição.

O conjunto de todos os harmônicos esféricos em $m+1$ variáveis é um subconjunto fundamental (total) de $X$, isto é,

$$
\overline{\left[\cup_{k=0}^{\infty} \mathcal{H}_{k}\left(S^{m}\right)\right]}=X
$$

Em particular, fixadas bases ortonormais $\left\{Y_{k 1}, Y_{k 2}, \ldots, Y_{k N(m, k)}\right\}$ de $\mathcal{H}_{k}\left(S^{m}\right), k=0,1, \ldots$ o conjunto $\left\{Y_{k l}: k \in \mathbb{N} ; l=1,2, \ldots, N(m, k)\right\}$ é um conjunto ortonormal completo de $L^{2}\left(S^{m}\right)$. O leitor deve ficar atento ao fato de utilizarmos a notação acima em vários pontos à frente, sem qualquer explicação adicional.

Toda função $f$ em $X$ possui uma expansão de Fourier da forma ([11])

$$
f \sim \sum_{k=0}^{\infty} \sum_{l=1}^{N(m, k)} \hat{f}(k, l) Y_{k l}
$$

onde o coeficiente de Fourier $\hat{f}(k, l)$ é dado por

$$
\hat{f}(k, l)=\frac{1}{\sigma_{m}} \int_{S^{m}} f \overline{Y_{k l}} d \sigma_{m} .
$$

Finalizamos essa seção estabelecendo e provando uma adaptação do Lema 4.2 de [5] ao contexto esférico.

Teorema 1.1.8. Seja $f \in L^{1}\left(S^{m}\right)$. Se

$$
\int_{S^{m}} f(x) g(x) d \sigma_{m}(x)=0, \quad g \in C\left(S^{m}\right) .
$$

então $f=0$.

Demonstração: Assuma que a fórmula do enunciado vale e seja $\epsilon>0$. Como $C\left(S^{m}\right)$ é denso em $L^{1}\left(S^{m}\right)$ ([10], p.70), existe $g_{\epsilon} \in C\left(S^{m}\right)$ tal que $\left\|f-g_{\epsilon}\right\|_{1}<\epsilon$. Como

$$
\begin{aligned}
\int_{S^{m}} g_{\epsilon}(x) g(x) d \sigma_{m} & =\int_{S^{m}}\left(g_{\epsilon}-f\right)(x) g(x) d \sigma_{m}(x)+\int_{S^{m}} f(x) g(x) d \sigma_{m}(x) \\
& =\int_{S^{m}}\left(g_{\epsilon}-f\right)(x) g(x) d \sigma_{m}(x), \quad g \in C\left(S^{m}\right),
\end{aligned}
$$


a Desigualdade de Hölder implica que

$$
\begin{aligned}
\left|\int_{S^{m}} g_{\epsilon}(x) g(x) d \sigma_{m}(x)\right| & \leq \int_{S^{m}}\left|\left(g_{\epsilon}-f\right)(x) \| g(x)\right| d \sigma_{m}(x) \\
& \leq\left\|f-g_{\epsilon}\right\|_{1}\|g\|_{\infty} \\
& <\epsilon\|g\|_{\infty} .
\end{aligned}
$$

Defina $K_{1}:=\left\{x \in S^{m} ; g_{\epsilon}(x) \geq \epsilon\right\}$ e $K_{2}:=\left\{x \in S^{m} ; g_{\epsilon}(x) \leq-\epsilon\right\}$. Como $K_{1}$ e $K_{2}$ são conjuntos compactos e disjuntos, o Lema de Urysohn ([25]) garante a existência de $h \in C\left(S^{m}\right)$ tal que $|h(x)| \leq 1, x \in S^{m}, h(x)=1, x \in K_{1}$ e $h(x)=-1, x \in K_{2}$. Fazendo $K=K_{1} \cup K_{2}$, encontramos

$$
\int_{S^{m}} g_{\epsilon}(x) h(x) d \sigma_{m}(x)=\int_{S^{m} \backslash K} g_{\epsilon}(x) h(x) d \sigma_{m}(x)+\int_{K} g_{\epsilon}(x) h(x) d \sigma_{m}(x)
$$

de modo que

$$
\begin{aligned}
\int_{K}\left|g_{\epsilon}(x)\right| d \sigma_{m}(x) & =\int_{K_{1}}\left|g_{\epsilon}(x)\right| d \sigma_{m}(x)+\int_{K_{2}}\left|g_{\epsilon}(x)\right| d \sigma_{m}(x) \\
& =\int_{K_{1}}\left|g_{\epsilon}(x) h(x)\right| d \sigma_{m}(x)+\int_{K_{2}}\left|g_{\epsilon}(x) h(x)\right| d \sigma_{m}(x) \\
& =\int_{K_{1}} g_{\epsilon}(x) h(x) d \sigma_{m}(x)+\int_{K_{2}} g_{\epsilon}(x) h(x) d \sigma_{m}(x) \\
& =\int_{K} g_{\epsilon}(x) h(x) d \sigma_{m}(x)
\end{aligned}
$$

Segue de (1.7) e (1.8) que

$$
\int_{K}\left|g_{\epsilon}(x)\right| d \sigma_{m}(x) \leq \epsilon+\int_{S^{m} \backslash K}\left|g_{\epsilon}(x)\right||h(x)| d \sigma_{m}(x) \leq \epsilon+\int_{S^{m} \backslash K}\left|g_{\epsilon}(x)\right| d \sigma_{m}(x) .
$$

Conseqüentemente,

$$
\begin{aligned}
\int_{S^{m}}\left|g_{\epsilon}(x)\right| d \sigma_{m}(x) & =\int_{K}\left|g_{\epsilon}(x)\right| d \sigma_{m}(x)+\int_{S^{m} \backslash K}\left|g_{\epsilon}(x)\right| d \sigma_{m}(x) \\
& \leq \epsilon+2 \int_{S^{m} \backslash K}\left|g_{\epsilon}(x)\right| d \sigma_{m}(x) \\
& \leq \epsilon+2 \epsilon \sigma_{m},
\end{aligned}
$$

pois $\left|g_{\epsilon}(x)\right| \leq \epsilon, x \in S^{m} \backslash K$. Portanto,

$$
\|f\|_{1} \leq\left\|f-g_{\epsilon}\right\|_{1}+\left\|g_{\epsilon}\right\|_{1} \leq 2 \epsilon+2 \epsilon \sigma_{m} .
$$

Como $\epsilon$ é arbitrário, a prova está completa. 


\subsection{O Teorema da Adição e a Fórmula de Funk-Hecke}

Nesta seção apresentaremos o Teorema da Adição e a Fórmula de Funk-Hecke. O Teorema da Adição é um resultado insubstituível na análise de vários problemas de natureza esférica. Ele surge quando se tenta determinar os elementos de $\mathbb{P}_{n}\left(S^{m}\right)$ que são zonais, isto é, que são invariantes por transformações ortogonais que fixam um determinado ponto de $S^{m}$. Sendo o Teorema da Adição um resultado clássico, sua prova será omitida (veja [22] e [23]).

Teorema 1.2.1. (Teorema da Adição) Seja $\left\{Y_{n 1}, Y_{n 2}, \ldots, Y_{n N(m, n)}\right\}$ uma base ortonormal de $\mathcal{H}_{n}\left(S^{m}\right)$. Então

$$
\sum_{l=1}^{N(m, n)} Y_{n l}(x) \overline{Y_{n l}(y)}=N(m, n) P_{n}^{m}(\langle x, y\rangle), \quad x, y \in S^{m},
$$

onde $P_{n}^{m}$ é o polinômio de Legendre de grau $k$ associado à dimensão $m+1$ e $\langle\cdot, \cdot\rangle$ é o produto interno usual de $\mathbb{R}^{m+1}$.

Observamos que a normalização do polinômio de Legendre é $P_{n}^{m}(1)=1$. Ainda, $P_{n}^{1}$ é o polinômio de Chebyshev de grau $n$ e $P_{n}^{2}$ é o polinômio de Legendre clássico de grau $n$. Os polinômios de Legendre podem ser escritos na forma

$$
P_{n}^{m}=\frac{C_{n}^{(m-1) / 2}}{C_{n}^{(m-1) / 2}(1)}, \quad m \geq 2,
$$

onde $C_{n}^{(m-1) / 2}$ é o polinômio de Gegenbauer de grau $n$ associado ao racional $(m-1) / 2$. Os polinômios de Gegenbauer $C_{n}^{\lambda}, \lambda>-1 / 2$, podem ser obtidos através da expansão em $z$ da função geradora $\left(1-2 z t+z^{2}\right)^{-\lambda}, z \in[-1,1]$. Precisamente,

$$
\sum_{k=0}^{\infty} C_{k}^{\lambda}(t) z^{k}=\frac{1}{\left(1-2 z t+z^{2}\right)^{\lambda}}, \quad t \in[-1,1] .
$$

Em particular, observamos que

$$
C_{n}^{(m-1) / 2}(1)=\frac{\Gamma(n+m-1)}{n ! \Gamma(m-1)} .
$$

Além disso, valem as relações ([34], p. 81,82),

$$
\frac{d}{d t} C_{n}^{(m-1) / 2}(t)=(m-1) C_{n-1}^{(m+1) / 2}(t)
$$

e

$$
\left(1-t^{2}\right) \frac{d^{2}}{d t^{2}} C_{n}^{(m-1) / 2}(t)-m t \frac{d}{d t} C_{n}^{(m-1) / 2}(t)+n(n+m-1) C_{n}^{(m-1) / 2}(t)=0
$$

Essas duas relações implicam no seguinte resultado: 
Proposição 1.2.2. Se $n \geq 1$, vale a seguinte igualdade:

$$
\frac{d}{d t}\left(-\frac{m-1}{n(n+m-1)}\left(1-t^{2}\right)^{m / 2} C_{n-1}^{(m+1) / 2}(t)\right)=\left(1-t^{2}\right)^{(m-2) / 2} C_{n}^{(m-1) / 2}(t) .
$$

Demonstração: Derivação por partes produz a fórmula

$$
\begin{aligned}
& \frac{d}{d t}\left(-\frac{m-1}{n(n+m-1)}\left(1-t^{2}\right)^{m / 2} C_{n-1}^{(m+1) / 2}(t)\right)= \\
& \quad=\frac{m-1}{n(n+m-1)}\left[m t\left(1-t^{2}\right)^{(m-2) / 2} C_{n-1}^{(m+1) / 2}(t)-\left(1-t^{2}\right)^{m / 2} \frac{d}{d t} C_{n-1}^{(m+1) / 2}(t)\right],
\end{aligned}
$$

enquanto que o uso de (1.9) e (1.10) nos leva a

$$
\begin{aligned}
& \frac{d}{d t}\left(-\frac{m-1}{n(n+m-1)}\left(1-t^{2}\right)^{m / 2} C_{n-1}^{(m+1) / 2}(t)\right)= \\
& \quad=\frac{1}{n(n+m-1)}\left(1-t^{2}\right)^{(m-2) / 2}\left[m t \frac{d}{d t} C_{n}^{(m-1) / 2}(t)-\left(1-t^{2}\right) \frac{d^{2}}{d t^{2}} C_{n}^{(m-1) / 2}(t)\right] \\
& \quad=\left(1-t^{2}\right)^{(m-2) / 2} C_{n}^{(m-1) / 2}(t) .
\end{aligned}
$$

Logo, a prova está completa.

No que segue, consideraremos o espaço das funções integráveis sobre $[-1,1]$ em relação à medida $w_{m}$ definida pela relação

$$
d w_{m}(t):=\left(1-t^{2}\right)^{(m-2) / 2} d t
$$

A norma em tal espaço é dada por

$$
\|K\|_{1, m}:=\frac{\sigma_{m-1}}{\sigma_{m}} \int_{-1}^{1}|K(t)| d w_{m}(t), \quad K \in L^{1, m}\left([-1,1], d w_{m}\right) .
$$

Não é difícil verificar que o conjunto $\left\{P_{k}^{m}: k=0,1, \ldots\right\}$ é um subconjunto ortogonal de $L^{1, m}\left([-1,1], d w_{m}\right)$, (ver [22]). Em particular, temos o seguinte resultado:

Proposição 1.2.3. Seja $k$ um inteiro não negativo. Então

$$
\int_{-1}^{1}\left(P_{k}^{m}(t)\right)^{2} d w_{m}(t)=\frac{\sigma_{m}}{\sigma_{m-1} N(m, k)}
$$

O próximo teorema descreve a versão mais elementar da Fórmula de Funk-Hecke. Essa fórmula transforma uma integral na esfera em uma integral no intervalo $[-1,1]$. Sua prova pode ser encontrada em [11] e [23].

Teorema 1.2.4. (Fórmula de Funk-Hecke) Seja $K:[-1,1] \rightarrow \mathbb{C}$ uma função tal que $\|K\|_{1, m}<\infty$ e $Y_{k} \in \mathcal{H}_{k}\left(S^{m}\right)$. Então

$$
\int_{S^{m}} K(\langle x, y\rangle) Y_{k}(y) d \sigma_{m}(y)=a_{k}^{m}(K) Y_{k}(x), \quad x \in S^{m},
$$

onde

$$
a_{k}^{m}(K)=\sigma_{m-1} \int_{-1}^{1} K(t) P_{k}^{m}(t) d w_{m}(t)
$$


A expressão que aparece no lado esquerdo da igualdade que define a Fórmula de FunkHecke, é um caso particular do conceito abaixo. Se $K$ é como no Teorema 1.2.4, o operador $f \in X \mapsto T_{K}(f) \in X$ dado por

$$
T_{K}(f)(x)=\frac{1}{\sigma_{m}} \int_{S^{m}} K(\langle x, y\rangle) f(y) d \sigma_{m}(y), \quad x \in S^{m},
$$

é chamado de convolução esférica gerada por $K$. Notemos que o Teorema 6.18 de [10] garante que, de fato, $T_{K}$ está bem definido. A notação mais comum para este operador, a qual será adotada aqui, é $T_{K}(f)=K * f$.

A proposição abaixo pode ser encontrada em ([3], p. 208).

Proposição 1.2.5. Sejam $f \in X$ e $K \in L^{1, m}\left([-1,1], d w_{m}\right)$. Então, vale a desigualdade $\|K * f\|_{X} \leq\|K\|_{1, m}\|f\|_{X}$.

Teorema 1.2.6. Seja $K \in L^{1, m}\left([-1,1], d w_{m}\right)$. Se $f, g \in X$ e o produto $(K * f) g$ é $\sigma_{m^{-}}$ integrável, então

$$
\int_{S^{m}}(K * f)(x) g(x) d \sigma_{m}(x)=\int_{S^{m}} f(x)(K * g)(x) d \sigma_{m}(x) .
$$

Demonstração: É uma aplicação direta do Teorema de Fubini ([33], p. 384).

Informações mais refinadas sobre o operador introduzido acima podem ser encontradas em [3]. Para aplicações em problemas de aproximação, ver [19]. No Capítulo 2, apresentaremos alguns resultados adicionais sobre convolução esférica que serão necessários para o completamento do trabalho.

\subsection{Fórmula de linearização de Dougall}

Nesta seção apresentaremos uma fórmula de linearização para polinômios de Legendre, a ser utilizada no Capítulo 3. Várias são as referências em que podemos encontrar informações mais detalhadas sobre o assunto, dentre elas, [1], [6],[9] e [13].

Antes de apresentarmos a fórmula de linearização de Dougall, precisaremos de alguns resultados sobre funções positivas definidas.

Definição 1.3.1. Uma função $f:[-1,1] \rightarrow \mathbb{C}$ contínua é positiva definida em $S^{m}$ se para todo $n \geq 1$ e qualquer escolha dos pontos $x_{1}, x_{2}, \ldots, x_{n}$ em $S^{m}$, a matriz $A_{n \times n}$ com entradas $a_{i j}=f\left(\left\langle x_{i}, x_{j}\right\rangle\right)$ é não negativa definida, isto é, se

$$
c A c^{T}=\sum_{i=1}^{n} \sum_{j=1}^{n} c_{i} f\left(\left\langle x_{i}, x_{j}\right\rangle\right) c_{j} \geq 0
$$

para todos os vetores $c=\left(c_{1}, c_{2}, \ldots, c_{n}\right) \in \mathbb{R}^{m}$. 
Os polinômios de Gegenbauer são exemplos de funções positivas definidas. Em particular, os polinômios de Legendre $P_{k}^{m}, k \in \mathbb{N}$, são funções positivas definidas. Isso segue diretamente da Fórmula da Adição, como observou Schoenberg em [30].

O prova do próximo teorema pode ser encontrada em [12].

Teorema 1.3.2. (Teorema do produto de Schur) $S e A=\left(a_{i j}\right)_{n \times n}$ e $B=\left(b_{i j}\right)_{n \times n}$ são matrizes não negativas definidas, então a matriz $C=\left(a_{i j} b_{i j}\right)_{n \times n}$ é não negativa definida.

Observemos que o Teorema de Schur garante que o produto de duas funções positivas definidas é positiva definida.

O próximo teorema nos dá uma caracterização das funções que são positivas definidas em $S^{m}$. Esse teorema é devido à I. J. Schoenberg e sua demonstração pode ser encontrada em [30].

Teorema 1.3.3. Uma função contínua $f:[-1,1] \rightarrow \mathbb{C}$ é positiva definida em $S^{m}$ se, e somente se, ela possui a seguinte representação:

$$
f(t)=\sum_{k=0}^{\infty} a_{k} C_{k}^{(m-1) / 2}(t)
$$

com $a_{k} \geq 0, k=0,1, \ldots$, e $\sum_{k=0}^{\infty} a_{k} C_{k}^{(m-1) / 2}(1)<\infty$.

Uma formulação da fórmula de linearização de Dougall é dada pelo seguinte teorema.

Teorema 1.3.4. Sejam $m \geq 2$ e, $k$ e i inteiros não negativos. Então

$$
P_{k}^{m}(t) P_{i}^{m}(t)=\sum_{j=0}^{k \wedge i} c(k, i, j) P_{k+i-2 j}^{m}(t), \quad t \in[-1,1],
$$

onde os coeficientes $c(k, i, j)$ são todos não negativos.

Demonstração: A prova de (1.12) está feita em [6]. Para mostrar que os coeficientes $c(k, i, j)$, $j=0, \ldots, k \wedge i$, são não negativos, começamos observando que como os polinômios de Legendre são funções positivas definidas, o Teorema do produto de Schur garante que o produto $P_{k}^{m} P_{i}^{m}$ é uma função positiva definida. Logo, pelo Teorema 1.3.3, $P_{k}^{m} P_{i}^{m}$ pode ser escrito como uma soma de polinômios de Legendre com coeficientes não negativos. Isso completa a prova do teorema.

Chamando $u:=k+i-2 j$, podemos escrever a Equação (1.12) da seguinte maneira:

$$
P_{k}^{m}(t) P_{i}^{m}(t)=\sum_{u=|k-i|}^{k+i} d(k, i, u) P_{u}^{m}(t)
$$

onde $d(k, i, u) \geq 0,|k-i| \leq u \leq k+i$. 
Teorema 1.3.5. Se $k$ e $i$ são inteiros não negativos, então $\sum_{j=|k-i|}^{k+i} d(k, i, j)=1$.

Demonstração: Basta tomar $t=1$ na igualdade (1.13).

O próximo resultado nos dá uma caracterização dos coeficientes da fórmula de linearização de Dougall.

Proposição 1.3.6. Sejam $k$, $i$ e $j$ inteiros não negativos tais que $|k-i| \leq j \leq k+i$. Então

$$
d(k, i, j)=\frac{\sigma_{m-1}}{\sigma_{m}} N(m, n) \int_{-1}^{1} P_{k}^{m}(t) P_{i}^{m}(t) P_{j}^{m}(t) d w_{m}(t) .
$$

Demonstração: Usando a fórmula de linearização dada acima segue que

$$
\begin{aligned}
\int_{-1}^{1} P_{k}^{m}(t) P_{i}^{m}(t) P_{j}^{m}(t) d w_{m}(t) & =\sum_{u=|k-i|}^{k+i} d(k, i, u) \int_{-1}^{1} P_{u}^{m}(t) P_{j}^{m}(t) d w_{m}(t) \\
& =d(k, i, j) \int_{-1}^{1}\left(P_{j}^{m}(t)\right)^{2} d w_{m}(t)
\end{aligned}
$$

Logo, a Proposição 1.2.3 implica que

$$
d(k, i, j)=\frac{\sigma_{m-1}}{\sigma_{m}} N(m, n) \int_{-1}^{1} P_{k}^{m}(t) P_{i}^{m}(t) P_{j}^{m}(t) d w_{m}(t),
$$

completando a prova da proposição.

Segue da proposição acima que

$$
d(k, i, j)=d(i, k, j)=\frac{N(m, j)}{N(m, i)} d(j, k, i) .
$$

Para finalizar essa seção calcularemos um dos coeficientes da fórmula de linearização de Dougall que será usado no Capítulo 3.

Proposição 1.3.7. Se $k \in \mathbb{N}$ então $d(k, k, 0)=N(m, k)^{-1}$.

Demonstração: Usando as Proposiçôes 1.3.6 e 1.2.3 temos que

$$
\begin{aligned}
d(k, k, 0) & =\frac{\sigma_{m-1}}{\sigma_{m}} N(m, 0) \int_{-1}^{1}\left(P_{k}^{m}(t)\right)^{2} P_{0}^{m}(t) d w_{m}(t) \\
& =\frac{\sigma_{m-1}}{\sigma_{m}} \int_{-1}^{1}\left(P_{k}^{m}(t)\right)^{2} d w_{m}(t) \\
& =\frac{1}{N(m, k)}
\end{aligned}
$$

Logo, a proposição está provada. 


\section{Capítulo}

\section{A derivada forte de Laplace-Beltrami e operadores associados}

O objetivo neste capítulo é introduzir a derivada forte de Laplace-Beltrami e alguns operadores associados a ela. Aparentemente esse conceito foi introduzido por Rudin em [28] no caso tri-dimensional, sem no entanto usar essa nomenclatura. Como estamos trabalhando sem fixar a dimensão, apresentaremos provas detalhadas para quase todos os resultados. Entretanto, observamos que, provas para alguns deles no caso tri-dimensional já estão disponíveis em [28] e [37]. A extensão de várias delas para o caso geral é não trivial. Na seção 2.8, definiremos o módulo de suavidade esférico que utilizaremos no último capítulo deste trabalho para analisar ordens de convergência dos processos aproximatórios que iremos discutir.

\subsection{A projeção esférica}

Esta seção contém propriedades básicas do operador que projeta o espaço $X$ sobre o subespaço $\mathcal{H}_{n}\left(S^{m}\right)$. O operador projeção $\mathcal{Y}_{n}: X \rightarrow X$ é definido por

$$
\mathcal{Y}_{n}(f)(x):=\frac{N(m, n)}{\sigma_{m}} \int_{S^{m}} P_{n}^{m}(\langle x, y\rangle) f(y) d \sigma_{m}(y), \quad x \in S^{m}, \quad n \in \mathbb{N} .
$$

Para ver que $\mathcal{Y}_{n}(f) \in \mathcal{H}_{n}\left(S^{m}\right), f \in X$, basta aplicarmos o Teorema da Adição. De fato,

$$
\begin{aligned}
\mathcal{Y}_{n}(f)(x) & =\frac{N(m, n)}{\sigma_{m}} \int_{S^{m}} P_{n}^{m}(\langle x, y\rangle) f(y) d \sigma_{m}(y) \\
& =\frac{1}{\sigma_{m}} \sum_{l=1}^{N(m, n)} Y_{n l}(x) \int_{S^{m}} f(y) \overline{Y_{n l}(y)} d \sigma_{m}(y) \\
& =\sum_{l=1}^{N(m, n)} \hat{f}(n, l) Y_{n l}(x), \quad x \in S^{m} .
\end{aligned}
$$

O fato de $\mathcal{Y}_{n}$ ser uma projeção segue do seguinte resultado mais geral. 
Proposição 2.1.1. Sejam $n, k \in \mathbb{N}$. Então

$$
\mathcal{Y}_{n} \circ \mathcal{Y}_{k}=\delta_{n k} \mathcal{Y}_{n}
$$

Demonstração: Seja $f \in X$. Usando a representação dada acima segue que

$$
\begin{aligned}
\mathcal{Y}_{n}\left(\mathcal{Y}_{k}(f)\right) & =\sum_{l=1}^{N(m, n)} \widehat{\mathcal{Y}_{k}(f)}(n, l) Y_{n l} \\
& =\sum_{l=1}^{N(m, n)}\left(\frac{1}{\sigma_{m}} \int_{S^{m}} \mathcal{Y}_{k}(f)(x) \overline{Y_{n l}(x)} d \sigma_{m}(x)\right) Y_{n l} \\
& =\sum_{l=1}^{N(m, n)} \sum_{j=1}^{N(m, k)} \hat{f}(k, j)\left(\frac{1}{\sigma_{m}} \int_{S^{m}} Y_{k j}(x) \overline{Y_{n l}(x)} d \sigma_{m}(x)\right) Y_{n l} \\
& =\delta_{n k} \sum_{l=1}^{N(m, n)} \hat{f}(n, l) Y_{n l} \\
& =\delta_{n k} \mathcal{Y}_{n}(f) .
\end{aligned}
$$

Isso prova a proposição.

É fácil ver que $\mathcal{Y}_{n}(0)=0, n \in \mathbb{N}$. A recíproca deste resultado é o conteúdo do próximo teorema.

Teorema 2.1.2. Seja $f \in X$. Se $\mathcal{Y}_{n}(f)=0, n \in \mathbb{N}$, então $f=0$.

Demonstração: Suponhamos que $\mathcal{Y}_{n}(f)=0, n \in \mathbb{N}$. Consideremos primeiramente os casos $X=C\left(S^{m}\right)$ e $X=L^{2}\left(S^{m}\right)$. Fixemos $n \in \mathbb{N}, l \in\{1,2, \ldots, N(m, n)\}$ e escolhamos um sistema fundamental $\left\{x_{1}, x_{2}, \ldots, x_{N(m, n)}\right\}$ em $S^{m}$ ( [23], p. 31-33). Então existem números complexos $\alpha_{n j} \in \mathbb{R}$ tais que ([35], Teorema 1.1.12, p.15)

$$
Y_{n l}(y)=\sum_{j=1}^{N(m, n)} \alpha_{n j} P_{n}^{m}\left(\left\langle x_{j}, y\right\rangle\right)
$$

Logo,

$$
\int_{S^{m}} Y_{n l}(y) f(y) d \sigma_{m}(y)=\sum_{j=1}^{N(m, n)} \alpha_{n j} \int_{S^{m}} f(y) P_{n}^{m}\left(\left\langle x_{j}, y\right\rangle\right) d \sigma_{m}(y)=0 .
$$

Como $\left\{Y_{n l}: n \in \mathbb{N} ; l=1,2, \ldots, N(m, n)\right\}$ é um conjunto ortonormal completo de $L^{2}\left(S^{m}\right)$ segue que $f=0$. Se $f \in L^{1}\left(S^{m}\right) \backslash L^{2}\left(S^{m}\right)$, então procedendo como acima temos que

$$
\int_{S^{m}} Y_{n l}(y) f(y) d \sigma_{m}(y)=0, \quad n \in \mathbb{N}, \quad l=1,2, \ldots, N(m, n) .
$$

Em vista do Teorema 1.1.8, para terminar a prova, é suficiente mostrar que

$$
\int_{S^{m}} f(y) h(y) d \sigma_{m}(y)=0, \quad h \in C\left(S^{m}\right) .
$$


Seja $p \in P\left(S^{m}\right)$. Como $p$ pode ser escrito como combinação linear de harmônicos esféricos segue que

$$
\int_{S^{m}} f(y) p(y) d \sigma_{m}(y)=0 .
$$

Se $h \in C\left(S^{m}\right)$, segue do Teorema da Aproximação de Weierstrass que existe uma seqüência $\left\{p_{n}\right\}_{n \in \mathbb{N}} \subset \mathbb{P}\left(S^{m}\right)$, tal que

$$
\lim _{n \rightarrow \infty}\left\|h-p_{n}\right\|_{\infty}=0
$$

Conseqüentemente,

$$
\begin{aligned}
\left|\int_{S^{m}} f(y) h(y) d \sigma_{m}(y)\right| & \leq\left|\int_{S^{m}} f(y)\left(h-p_{n}\right)(y) d \sigma_{m}(y)\right|+\left|\int_{S^{m}} f(y) p_{n}(y) d \sigma_{m}(y)\right| \\
& \leq \int_{S^{m}}|f(y)|\left|\left(h-p_{n}\right)(y)\right| d \sigma_{m}(y) \\
& \leq\left\|h-p_{n}\right\|_{\infty} \int_{S^{m}}|f(y)| d \sigma_{m}(y) .
\end{aligned}
$$

Tomando o limite quando $n \rightarrow \infty$ na desigualdade acima vem que

$$
\int_{S^{m}} f(y) h(y) d \sigma_{m}(y)=0
$$

completando a prova do teorema.

Proposição 2.1.3. Seja $n$ um inteiro não-negativo. Valem as seguintes propriedades:

(i) $\mathcal{Y}_{n}\left(Y_{k}\right)=\delta_{n k} Y_{n}, Y_{k} \in \mathcal{H}_{k}\left(S^{m}\right)$;

(ii) $\left|\mathcal{Y}_{n}(f)(x)\right| \leq N(m, n)\|f\|_{X}, f \in X, x \in S^{m}$;

(iii) $\left\|\mathcal{Y}_{n}(f)\right\|_{X} \leq N(m, n)\|f\|_{X}, f \in X$.

Demonstração: Para provar $(i)$ é suficiente considerar um elemento básico de $\mathcal{H}_{k}\left(S^{m}\right)$ e usar o Teorema da Adição. Logo, se $l \in\{1,2, \ldots, N(m, k)\}$

$$
\begin{aligned}
\mathcal{Y}_{n}\left(Y_{k l}\right)(x) & =\frac{N(m, n)}{\sigma_{m}} \int_{S^{m}} Y_{k l}(y) P_{n}^{m}(\langle x, y\rangle) d \sigma_{m}(y) \\
& =\sum_{j=1}^{N(m, n)} Y_{n j}(x) \frac{1}{\sigma_{m}} \int_{S^{m}} Y_{k l}(y) \overline{Y_{n j}(y)} d \sigma_{m}(y) \\
& =\delta_{n k} Y_{n l}(x), \quad x \in S^{m} .
\end{aligned}
$$

Para provar o item $(i i)$, consideremos primeiramente os casos em que $X=C\left(S^{m}\right)$ e $X=$ $L^{1}\left(S^{m}\right)$. Se $f \in X$, então como $\left|P_{n}^{m}(t)\right| \leq 1,-1 \leq t \leq 1$, segue que

$$
\begin{aligned}
\left|\mathcal{Y}_{n}(f)(x)\right| & =\left|\frac{N(m, n)}{\sigma_{m}} \int_{S^{m}} P_{n}^{m}(\langle x, y\rangle) f(y) d \sigma_{m}(y)\right| \\
& \leq \frac{N(m, n)}{\sigma_{m}} \int_{S^{m}}\left|P_{n}^{m}(\langle x, y\rangle) \| f(y)\right| d \sigma_{m}(y) \\
& \leq N(m, n)\|f\|_{X}, \quad x \in S^{m} .
\end{aligned}
$$


Para o caso em que $X=L^{p}\left(S^{m}\right), 1<p<\infty$, sejam $f \in X$ e $p^{\prime}$ o expoente conjugado de $p$. Como $P_{n}^{m}(\langle x, \cdot\rangle) \in L^{p^{\prime}}\left(S^{m}\right), x \in S^{m}$, a desigualdade de Hölder e o fato de que $\left|P_{n}^{m}(t)\right| \leq 1$, $-1 \leq t \leq 1$, implicam que

$$
\begin{aligned}
\left|\mathcal{Y}_{n}(f)(x)\right| & \leq \frac{N(m, n)}{\sigma_{m}} \int_{S^{m}}\left|P_{n}^{m}(\langle x, y\rangle) \| f(y)\right| d \sigma_{m}(y) \\
& \leq N(m, n)\left\|P_{n}^{m}(\langle x, \cdot\rangle)\right\|_{p^{\prime}}\|f\|_{p} \\
& \leq N(m, n)\|f\|_{p}, \quad x \in S^{m}
\end{aligned}
$$

O item (iii) segue diretamente de (ii). Assim, a prova da proposição está completa.

Proposição 2.1.4. Sejam $n$ um inteiro não-negativo, $f \in X$ e $K \in L^{1, m}\left([-1,1], d w_{m}\right)$. Então $\mathcal{Y}_{n}(K * f)=K^{\wedge}(n) \mathcal{Y}_{n}(f)$, onde $K^{\wedge}(n)$ é o n-ésimo coeficiente de Fourier-Legendre de $K$, isto é,

$$
K^{\curlyvee}(n):=\frac{\sigma_{m-1}}{\sigma_{m}} \int_{-1}^{1} K(t) P_{n}^{m}(t) d w_{m}(t) .
$$

Demonstração: Usando o Teorema da Adição temos que

$$
\begin{aligned}
\mathcal{Y}_{n}(K * f)(x) & =\frac{N(m, n)}{\sigma_{m}} \int_{S^{m}} P_{n}^{m}(\langle x, y\rangle)(K * f)(y) d \sigma_{m}(y) \\
& =\frac{1}{\sigma_{m}} \sum_{l=1}^{N(m, n)} Y_{n l}(x) \int_{S^{m}} \overline{Y_{n l}(y)}(K * f)(y) d \sigma_{m}(y), \quad x \in S^{m} .
\end{aligned}
$$

O Teorema de Fubini ([33], p. 384) e a Fórmula de Funk-Hecke implicam que $\left(x \in S^{m}\right)$

$$
\begin{aligned}
\mathcal{Y}_{n}(K * f)(x) & =\frac{1}{\sigma_{m}^{2}} \sum_{l=1}^{N(m, n)} Y_{n l}(x) \int_{S^{m}} \overline{Y_{n l}(y)}\left(\int_{S^{m}} K(\langle y, z\rangle) f(z) d \sigma_{m}(z)\right) d \sigma_{m}(y) \\
& =\frac{1}{\sigma_{m}^{2}} \sum_{l=1}^{N(m, n)} Y_{n l}(x) \int_{S^{m}} f(z)\left(\int_{S^{m}} K(\langle y, z\rangle) \overline{Y_{n l}(y)} d \sigma_{m}(y)\right) d \sigma_{m}(z) \\
& =\frac{\sigma_{m-1}}{\sigma_{m}^{2}} \sum_{l=1}^{N(m, n)} Y_{n l}(x) \int_{S^{m}} f(z)\left(\int_{-1}^{1} K(t) P_{n}^{m}(t) d w_{m}(t)\right) \overline{Y_{n l}(z)} d \sigma_{m}(z) .
\end{aligned}
$$

Logo, usando a representação dada em (2.1), obtemos

$$
\mathcal{Y}_{n}(K * f)(x)=K^{\curlywedge}(n) \sum_{l=1}^{N(m, n)} \hat{f}(n, l) Y_{n l}(x)=K^{\curlywedge}(n) \mathcal{Y}_{n}(f)(x), \quad x \in S^{m}
$$

Isso completa a prova.

Observação 2.1.5. Na prova da proposição acima, no caso $X=L^{1}\left(S^{m}\right)$, a igualdade que se obtem é de fato

$$
\mathcal{Y}_{n}(K * f)(x)=K^{\curlyvee}(n) \mathcal{Y}_{n}(f)(x), \quad x \in S^{m} \backslash \Lambda, \quad n \in \mathbb{N},
$$

onde $\sigma_{m}(\Lambda)=0$. Como $\mathcal{Y}_{n}(K * f), K^{\curlyvee}(n) \mathcal{Y}_{n}(f) \in \mathcal{H}_{n}\left(S^{m}\right)$, tal igualdade acaba sendo válida para todo $x \in S^{m}$. 


\subsection{O operador translação esférica}

Nesta seção, introduziremos o operador translação esférica, provaremos algumas de suas propriedades principais e também alguns resultados relacionando a translação esférica à projeção esférica.

A translação esférica é mais uma noção que foi introduzida por Rudin em seu famoso artigo [28]. Novamente, apenas o caso tri-dimensional foi considerado. Mais tarde, este conceito foi explorado um pouco mais em [3], visando a aplicação do mesmo no estudo de problemas de saturação em esferas. Mais recentemente, tal conceito reapareceu como um importante ingrediente na definição de vários módulos de suavidade para funções definidas em esferas $([4])$.

Para $t \in(-1,1)$, a translação esférica $S_{t}^{m}$ é um operador linear positivo sobre $X$, definido pela expressão

$$
S_{t}^{m}(f)(x)=\frac{1}{\sigma_{m-1}\left(1-t^{2}\right)^{(m-1) / 2}} \int_{\langle x, y\rangle=t} f(y) d y, \quad x \in S^{m}, \quad f \in X
$$

onde $d y$ denota o elemento de medida usual da seção esférica $\mathbb{S}_{x}^{m, t}:=\left\{y \in S^{m}:\langle x, y\rangle=t\right\}$, (ver figura 2.1 na próxima página). Dessa forma, $S_{t}^{m}(f)(x)$ pode ser interpretado como a média de $f$ sobre a superfície de uma subesfera $m$-dimensional de raio $\left(1-t^{2}\right)^{1 / 2}$. As referências [3] e [35] contém algumas informações básicas sobre esse operador.

A justificativa de alguns resultados sobre a translação esférica usa o resultado descrito pelo teorema abaixo. Uma prova do mesmo pode ser encontrada em ([23], p. 30).

Teorema 2.2.1. Se $t \in(-1,1)$ e $x \in S^{m}$, então

$$
\int_{\langle x, y\rangle=t} Y_{k}(y) d y=\sigma_{m-1}\left(1-t^{2}\right)^{(m-1) / 2} P_{k}^{m}(t) Y_{k}(x), \quad Y_{k} \in H_{k}\left(S^{m}\right) .
$$

Uma conseqüência imediata do teorema acima, é a fórmula

$$
\sigma_{m}\left(\mathbb{S}_{x}^{m, t}\right)=\sigma_{m-1}\left(1-t^{2}\right)^{(m-1) / 2}
$$

que calcula a medida $\sigma_{m}\left(\mathbb{S}_{x}^{m, t}\right)$ de $\mathbb{S}_{x}^{m, t}$. Observamos também que

$$
S_{t}^{m}(f)=\frac{\sigma_{m}}{\sigma_{m-1}\left(1-t^{2}\right)^{(m-1) / 2}} K_{t} * f
$$

onde

$$
K_{t}(\langle x, y\rangle)= \begin{cases}1, & \langle x, y\rangle=t \\ 0, & \langle x, y\rangle \neq t\end{cases}
$$

A figura 2.1 a seguir mostra a seção esférica $\mathbb{S}_{x}^{m, t}$ no caso da esfera tridimensional $S^{2}$. 


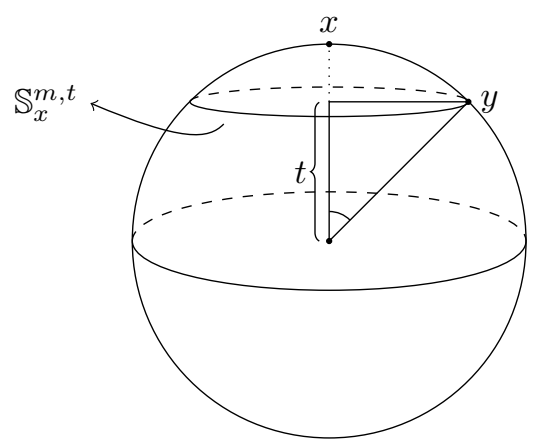

Figura 2.1: Seção esférica $\mathbb{S}_{x}^{m, t}$

O próximo resultado nos fornece mais uma propriedade da translação esférica que será usada na Proposição 2.2.7.

Proposição 2.2.2. Sejam $t \in(-1,1)$ e $f, g \in X$. Então

$$
\int_{S^{m}} S_{t}^{m}(f)(x) g(x) d \sigma_{m}(x)=\int_{S^{m}} f(x) S_{t}^{m}(g)(x) d \sigma_{m}(x) .
$$

Demonstração: Usando a observação acima e o Teorema 1.2.6 segue que

$$
\begin{aligned}
\int_{S^{m}} S_{t}^{m}(f)(x) g(x) d \sigma_{m}(x) & =\frac{\sigma_{m}}{\sigma_{m-1}\left(1-t^{2}\right)^{(m-1) / 2}} \int_{S^{m}}\left(K_{t} * f\right)(x) g(x) d \sigma_{m}(x) \\
& =\frac{\sigma_{m}}{\sigma_{m-1}\left(1-t^{2}\right)^{(m-1) / 2}} \int_{S^{m}} f(x)\left(K_{t} * g\right)(x) d \sigma_{m}(x) \\
& =\int_{S^{m}} f(x) S_{t}^{m}(g)(x) d \sigma_{m}(x) .
\end{aligned}
$$

Logo, a prova está completa.

Observando que $K_{t}$ é uma função a valores reais, podemos mostrar de maneira análoga à prova da proposição anterior, o seguinte resultado.

Proposição 2.2.3. Se $t \in(-1,1)$ e $f, g \in X$, então $\left\langle S_{t}^{m}(f), g\right\rangle_{2}=\left\langle f, S_{t}^{m}(g)\right\rangle_{2}$.

Proposição 2.2.4. Seja $t \in(-1,1)$. Valem as seguintes propriedades:

(i) $\left\|S_{t}^{m}(f)\right\|_{X} \leq\|f\|_{X}, f \in X$;

(ii) $\left\|S_{t}^{m}\right\|_{X}=1$.

Demonstração: Para provar o item $(i)$, seja $f \in X$. Usando (2.4) e a Proposição 1.2.5 obtemos

$$
\begin{aligned}
\left\|S_{t}^{m}(f)\right\|_{X} & =\frac{\sigma_{m}}{\sigma_{m-1}\left(1-t^{2}\right)^{(m-1) / 2}}\left\|K_{t} * f\right\|_{X} \\
& \leq \frac{\sigma_{m}}{\sigma_{m-1}\left(1-t^{2}\right)^{(m-1) / 2}}\left\|K_{t}\right\|_{1, m}\|f\|_{X} .
\end{aligned}
$$


A não-negatividade de $K_{t}$ e a Fórmula de Funk-Hecke implicam que

$$
\begin{aligned}
\left\|K_{t}\right\|_{1, m} & =\frac{\sigma_{m-1}}{\sigma_{m}} \int_{-1}^{1} K_{t}(u) d w_{m}(u) \\
& =\frac{1}{\sigma_{m}} \int_{S^{m}} K_{t}(\langle x, y\rangle) d \sigma_{m}(y) \\
& =\frac{1}{\sigma_{m}} \int_{\langle x, y\rangle=t} d y \\
& =\frac{\sigma_{m-1}}{\sigma_{m}}\left(1-t^{2}\right)^{(m-1) / 2},
\end{aligned}
$$

onde $x \in S^{m}$. Logo, $\left\|S_{t}^{m}(f)\right\|_{X} \leq\|f\|_{X}$. Tomando-se $f_{0} \equiv 1$, temos que $\left\|S_{t}^{m}\left(f_{0}\right)\right\|_{X}=1=$ $\left\|f_{0}\right\|_{X}$. Assim, $\left\|S_{t}^{m}\right\|_{X}=1$.

$\mathrm{Na}$ prova do próximo resultado, utilizaremos um lema técnico, a saber, a desigualdade de Minkowski para integrais. Apenas enunciaremos aqui tal desigualdade, já que uma prova detalhada pode ser encontrada em ([10], p. 194).

Lema 2.2.5. Sejam $(Z, \mathcal{M}, \mu)$ e $(W, \mathcal{N}, \nu)$ espaços de medida $\sigma$-finita, e seja $\phi$ uma função $\mathcal{M} \otimes \mathcal{N}$-mensurável sobre $Z \times W$. Valem as seguintes desigualdades:

(i) Se $\phi \geq 0$ e $1 \leq p<\infty$, então

$$
\left(\int_{Z}\left(\int_{W} \phi(z, w) d \nu(w)\right)^{p} d \mu(z)\right)^{1 / p} \leq \int_{W}\left(\int_{Z} \phi(z, w)^{p} d \mu(z)\right)^{1 / p} d \nu(w) ;
$$

(ii) Se $1 \leq p \leq \infty, \phi(\cdot, w) \in L^{p}(Z, \mu)$ quase sempre, e a aplicação $w \in W \mapsto\|\phi(\cdot, w)\|_{p}$ pertence a $L^{1}(W, \nu)$, então para quase todo $z \in Z$, a função $\phi(z, \cdot)$ pertence a $L^{1}(W, \nu)$, a função

$$
z \in Z \mapsto \int_{W} \phi(z, w) d \nu(w)
$$

pertence a $L^{p}(Z, \mu)$, e vale a desigualdade

$$
\left\|\int_{W} \phi(\cdot, w) d \mu(w)\right\|_{p} \leq \int_{W}\|\phi(\cdot, w)\|_{p} d \mu(w) .
$$

Proposição 2.2.6. Seja $t \in(-1,1)$. Valem as seguintes propriedades:

(i) Se $f \in X$, então $\lim _{t \rightarrow 1^{-}}\left\|f-S_{t}^{m}(f)\right\|_{X}=0$;

(ii) $S_{t}^{m}\left(Y_{k}\right)=P_{k}^{m}(t) Y_{k}, Y_{k} \in \mathcal{H}_{k}\left(S^{m}\right), k=0,1, \ldots$;

(iii) $S_{t}^{m}\left(P_{k}^{m}(\langle\cdot, y\rangle)\right)=P_{k}^{m}(t) P_{k}^{m}(\langle\cdot, y\rangle), y \in S^{m}, k=0,1, \ldots$

Demonstração: Seja $f \in X$. Para provar $(i)$ primeiro escrevemos

$$
\begin{aligned}
\left|f(x)-S_{t}^{m}(f)(x)\right| & =\frac{1}{\sigma_{m-1}\left(1-t^{2}\right)^{(m-1) / 2}}\left|\int_{\langle x, y\rangle=t} f(x)-f(y) d y\right| \\
& \leq \frac{1}{\sigma_{m-1}\left(1-t^{2}\right)^{(m-1) / 2}} \int_{\langle x, y\rangle=t}|f(x)-f(y)| d y, \quad x \in S^{m} .
\end{aligned}
$$


No caso contínuo, a desigualdade acima implica que

$$
\left\|f-S_{t}^{m}(f)\right\|_{\infty} \leq \sup _{x \in S^{m}} \sup _{y \in \mathbb{S}_{x}^{m, t}}|f(x)-f(y)| .
$$

Se $t \rightarrow 1^{-}$, então $\langle x-y, x-y\rangle \rightarrow 0$, o que implica que $y \rightarrow x$. Daí, a continuidade de $f$ garante que $\lim _{t \rightarrow 1^{-}}\left\|f-S_{t}^{m}(f)\right\|_{\infty}=0$. No caso $X=L^{p}\left(S^{m}\right)$, primeiro usamos a desigualdade de Minkowski para integrais, para obter

$$
\left\|f-S_{t}^{m}(f)\right\|_{p} \leq \frac{1}{\sigma_{m-1}\left(1-t^{2}\right)^{(m-1) / 2}} \int_{\langle x, y\rangle=t}\|f(\cdot)-f(y)\|_{p} d y .
$$

Como a integral de uma função de $L^{1}\left(S^{m}\right)$ é absolutamente contínua, dado $\epsilon>0$, existe $\delta>0$ tal que

$$
\|f(\cdot)-f(y)\|_{p}<\epsilon, \quad \sigma_{m}\left(\mathbb{S}_{x}^{m, t}\right)<\delta
$$

Portanto,

$$
\|f(\cdot)-f(y)\|_{p}<\epsilon, \quad|t|>\left(1-\left(\frac{\delta}{\sigma_{m-1}}\right)^{2 /(m-1)}\right)^{1 / 2} .
$$

Logo, a condição $(i)$ segue. Se $Y_{k} \in \mathcal{H}_{k}\left(S^{m}\right)$, então o Teorema 2.2.1 implica que

$$
S_{t}^{m}\left(Y_{k}\right)(x)=\frac{1}{\sigma_{m-1}\left(1-t^{2}\right)^{(m-1) / 2}} \int_{\langle x, y\rangle=t} Y_{k}(y) d y=P_{k}^{m}(t) Y_{k}(x), \quad x \in S^{m} .
$$

Isso prova o item (ii). Para provar (iii), usamos o Teorema da Adição e a linearidade de $S_{t}^{m}$, para obter

$$
\begin{aligned}
S_{t}^{m}\left(P_{k}^{m}(\langle\cdot, y\rangle)\right)(x) & =\frac{1}{N(m, k)} S_{t}^{m}\left(\sum_{l=1}^{N(m, k)} Y_{k l}(x) \overline{Y_{k l}(y)}\right) \\
& =\frac{1}{N(m, k)} \sum_{l=1}^{N(m, k)} S_{t}^{m}\left(Y_{k l}\right)(x) \overline{Y_{k l}(y)} \\
& =\frac{P_{k}^{m}(t)}{N(m, k)} \sum_{l=1}^{N(m, k)} Y_{k l}(x) \overline{Y_{k l}(y)} \\
& =P_{k}^{m}(t) P_{k}^{m}(\langle x, y\rangle), \quad x, y \in S^{m}, \quad k=0,1, \ldots
\end{aligned}
$$

Isto conclui a prova da proposição.

Na proposição abaixo, computamos a composição entre o operador projeção e a translação.

Proposição 2.2.7. Se n é um inteiro não-negativo e $t \in(-1,1)$, então $\mathcal{Y}_{n} \circ S_{t}^{m}=P_{n}^{m}(t) \mathcal{Y}_{n}$.

Demonstração: Sejam $n \in \mathbb{N}$ e $t \in(-1,1)$. Da Proposição 2.2.2, temos que

$$
\begin{aligned}
\left(\mathcal{Y}_{n} \circ S_{t}^{m}\right)(f)(x) & =\frac{N(m, n)}{\sigma_{m}} \int_{S^{m}} P_{n}^{m}(\langle x, y\rangle) S_{t}^{m}(f)(y) d \sigma_{m}(y) \\
& =\frac{N(m, n)}{\sigma_{m}} \int_{S^{m}} S_{t}^{m}\left(P_{n}^{m}(\langle x, \cdot\rangle)\right)(y) f(y) d \sigma_{m}(y), \quad x \in S^{m} .
\end{aligned}
$$


Mas, usando o Teorema da Adição e o Teorema 2.2.1, vem que

$$
\begin{aligned}
S_{t}^{m}\left(P_{n}^{m}(\langle x, \cdot\rangle)\right)(y) & =\frac{1}{\sigma_{m-1}\left(1-t^{2}\right)^{(m-1) / 2}} \int_{\langle y, z\rangle=t} P_{n}^{m}(\langle x, z\rangle) d z \\
& =\frac{1}{\sigma_{m-1}\left(1-t^{2}\right)^{(m-1) / 2} N(m, n)} \sum_{l=1}^{N(m, n)} Y_{n l}(x) \int_{\langle y, z\rangle=t} \overline{Y_{n l}(z)} d z \\
& =\frac{P_{n}^{m}(t)}{N(m, n)} \sum_{l=1}^{N(m, n)} Y_{n l}(x) \overline{Y_{n l}(y)} \\
& =P_{n}^{m}(t) P_{n}^{m}(\langle x, y\rangle), \quad x, y \in S^{m}
\end{aligned}
$$

Logo, deduzimos que $\left(x \in S^{m}\right)$

$$
\left(\mathcal{Y}_{n} \circ S_{t}^{m}\right)(f)(x)=\frac{N(m, n)}{\sigma_{m}} P_{n}^{m}(t) \int_{S^{m}} P_{n}^{m}(\langle x, y\rangle) f(y) d \sigma_{m}(y)=P_{n}^{m}(t) \mathcal{Y}_{n}(f)(x),
$$

e a proposição segue.

Para $t \in(-1,1)$, o operador diferença esférica é o operador linear dado por

$$
\Delta_{t}:=I-S_{t}^{m}
$$

onde $I: X \rightarrow X$ é o operador identidade. Indutivamente, a r-ésima diferença esférica é definida por

$$
\Delta_{t}^{r}:=\Delta_{t} \circ \Delta_{t}^{r-1}, \quad r=2,3, \ldots
$$

Devido à Proposição 2.2.6-(i), o operador diferença esférica é usado para definir vários módulos de suavidade para funções definidas na esfera.

Proposição 2.2.8. Sejam $r$ um inteiro positivo e $t \in(-1,1)$. Valem as seguintes propriedades:

(i) $\left\|\Delta_{t}^{r}(f)\right\|_{X} \leq 2^{r}\|f\|_{X}, f \in X ;$

(ii) $\lim _{t \rightarrow 1^{-}}\left\|\Delta_{t}^{r}(f)\right\|_{X}=0, f \in X$;

(iii) $\mathcal{Y}_{n} \circ \Delta_{t}^{r}=\left(1-P_{n}^{m}(t)\right)^{r} \mathcal{Y}_{n}, n=0,1, \ldots$

Demonstração: Da Proposição 2.2.4 temos que $\left\|S_{t}^{m}(f)\right\|_{X} \leq\|f\|_{X}, f \in X$. Logo,

$$
\left\|\Delta_{t}(f)\right\|_{X}=\left\|f-S_{t}^{m}(f)\right\|_{X} \leq\|f\|_{X}+\left\|S_{t}^{m}(f)\right\|_{X} \leq 2\|f\|_{X}, \quad f \in X .
$$

Portanto,

$$
\left\|\Delta_{t}^{r}(f)\right\|_{X}=\left\|\Delta_{t}\left(\Delta_{t}^{r-1}(f)\right)\right\|_{X} \leq 2\left\|\Delta_{t}^{r-1}(f)\right\|_{X} \leq \ldots \leq 2^{r}\|f\|_{X}, \quad f \in X
$$

Por outro lado, a Proposição 2.2.6-(i) implica que

$$
\lim _{t \rightarrow 1^{-}}\left\|\Delta_{t}^{r}(f)\right\|_{X} \leq 2^{r-1} \lim _{t \rightarrow 1^{-}}\left\|\Delta_{t}(f)\right\|_{X}=0, \quad f \in X .
$$


Logo, $\lim _{t \rightarrow 1^{-}}\left\|\Delta_{t}^{r}(f)\right\|_{X}=0, f \in X$. Para provar (iii), consideremos primeiramente o caso $r=1$. A linearidade de $\mathcal{Y}_{n}$ e a Proposição 2.2 .7 implicam que

$$
\mathcal{Y}_{n}\left(\Delta_{t}(f)\right)=\mathcal{Y}_{n}(f)-\mathcal{Y}_{n}\left(S_{t}^{m}(f)\right)=\left(1-P_{n}^{m}(t)\right) \mathcal{Y}_{n}(f), \quad n \in \mathbb{N}, \quad f \in X
$$

Assim, para $r>1$, temos que

$$
\begin{aligned}
\mathcal{Y}_{n}\left(\Delta_{t}^{r}(f)\right) & =\mathcal{Y}_{n}\left(\Delta_{t}\left(\Delta_{t}^{r-1}\right)(f)\right) \\
& =\left(1-P_{n}^{m}(t)\right) \mathcal{Y}_{n}\left(\Delta_{t}^{r-1}(f)\right) \\
& \vdots \\
& =\left(1-P_{n}^{m}(t)\right)^{r} \mathcal{Y}_{n}(f), \quad n \in \mathbb{N}, \quad f \in X .
\end{aligned}
$$

A prova está completa.

Proposição 2.2.9. Se $t, h \in(-1,1)$, então $S_{t}^{m} \circ S_{h}^{m}=S_{h}^{m} \circ S_{t}^{m}$.

Demonstração: Seja $f \in X$. A Proposição 2.2.7 implica que

$$
\begin{aligned}
\mathcal{Y}_{n}\left(\left(S_{t}^{m} \circ S_{h}^{m}\right)(f)\right) & =P_{n}^{m}(t) \mathcal{Y}_{n}\left(S_{h}^{m}(f)\right) \\
& =P_{n}^{m}(t) P_{n}^{m}(h) \mathcal{Y}_{n}(f) \\
& =P_{n}^{m}(h) \mathcal{Y}_{n}\left(S_{t}^{m}(f)\right) \\
& =\mathcal{Y}_{n}\left(\left(S_{h}^{m} \circ S_{t}^{m}\right)(f)\right), \quad n=0,1, \ldots
\end{aligned}
$$

Logo, o Teorema 2.1.2 garante que $S_{t}^{m} \circ S_{h}^{m}=S_{h}^{m} \circ S_{t}^{m}$.

O teorema abaixo estabelece uma relação entre a convolução esférica e a translação esférica.

Teorema 2.2.10. Se $K \in L^{1, m}\left([-1,1], d w_{m}\right)$ e $f \in X$, então

$$
K * f(x)=\frac{\sigma_{m-1}}{\sigma_{m}} \int_{-1}^{1} K(t) S_{t}^{m}(f)(x) d w_{m}(t), \quad x \in S^{m} .
$$

Demonstração: Sejam $K \in L^{1, m}\left([-1,1], d w_{m}\right)$ e $f \in X$. Usando a Proposição 2.1.4 segue que

$$
\mathcal{Y}_{n}(K * f)(x)=K^{\curlyvee}(n) \mathcal{Y}_{n}(f)(x), \quad x \in S^{m}, \quad n \in \mathbb{N} .
$$

Para concluir a prova, definimos

$$
K_{f}(x):=\frac{\sigma_{m-1}}{\sigma_{m}} \int_{-1}^{1} K(t) S_{t}^{m}(f)(x) d w_{m}(t), \quad x \in S^{m},
$$

e calculamos $\mathcal{Y}_{n}\left(K_{f}\right)$. Mudando a ordem de integração temos que $\left(x \in S^{m}\right)$

$$
\begin{aligned}
\mathcal{Y}_{n}\left(K_{f}\right)(x) & =\frac{\sigma_{m-1}}{\sigma_{m}^{2}} N(m, n) \int_{S^{m}} P_{n}^{m}(\langle x, y\rangle)\left(\int_{-1}^{1} K(t) S_{t}^{m}(f)(y) d w_{m}(t)\right) d \sigma_{m}(y) \\
& =\frac{\sigma_{m-1}}{\sigma_{m}^{2}} N(m, n) \int_{-1}^{1} K(t)\left(\int_{S^{m}} P_{n}^{m}(\langle x, y\rangle) S_{t}^{m}(f)(y) d \sigma_{m}(y)\right) d w_{m}(t),
\end{aligned}
$$


enquanto que a Proposição 2.2.2 e o Teorema 2.2.6-(iii) implicam que

$$
\begin{aligned}
\mathcal{Y}_{n}\left(K_{f}\right)(x) & =\frac{\sigma_{m-1}}{\sigma_{m}^{2}} N(m, n) \int_{-1}^{1} K(t)\left(\int_{S^{m}} S_{t}^{m}\left(P_{n}^{m}(\langle x, \cdot\rangle)\right)(y) f(y) d \sigma_{m}(y)\right) d w_{m}(t) \\
& =\frac{\sigma_{m-1}}{\sigma_{m}^{2}} N(m, n) \int_{-1}^{1} K(t) P_{n}^{m}(t)\left(\int_{S^{m}} P_{n}^{m}(\langle x, y\rangle) f(y) d \sigma_{m}(y)\right) d w_{m}(t) \\
& =K^{\widehat{\imath}}(n) \mathcal{Y}_{n}(f)(x), \quad x \in S^{m}, \quad n \in \mathbb{N} .
\end{aligned}
$$

Logo, o Teorema 2.1.2 garante que $K * f=K_{f}$, completando a prova do teorema.

\subsection{A derivada forte de Laplace-Beltrami}

Nesta seção, introduziremos o conceito de derivada forte de Laplace-Beltrami e deduziremos suas propriedades básicas.

Definição 2.3.1. Dizemos que uma função $f \in X$ é diferenciável no sentido de LaplaceBeltrami quando existir uma função $D^{1}(f) \in X$ tal que

$$
\lim _{t \rightarrow 1^{-}}\left\|\frac{\Delta_{t}(f)}{1-t}-D^{1}(f)\right\|_{X}=0
$$

$D^{1}(f)$ é chamada a primeira derivada forte de Laplace-Beltrami de $f$.

Derivadas de ordem superior são definidas indutivamente por

$$
D^{r}:=D^{1} \circ D^{r-1}, \quad r=2,3, \ldots
$$

Denotamos o conjunto das funções diferenciáveis nesse sentido por $W_{X}^{r}$, isto é,

$$
W_{X}^{r}:=\left\{f \in X: D^{r}(f) \in X\right\}, \quad r=1,2, \ldots
$$

Observamos que $0 \in W_{X}^{r}$ e que $D^{r}(0)=0, r=1,2, \ldots$

Proposição 2.3.2. Sejam $k$ um inteiro não-negativo e $r$ um inteiro positivo. Valem as seguintes propriedades:

(i) $\mathcal{H}_{k}\left(S^{m}\right) \subset W_{X}^{r}$;

(ii) $D^{r}\left(Y_{k}\right)=k^{r}(k+m-1)^{r} m^{-r} Y_{k}, Y_{k} \in \mathcal{H}_{k}\left(S^{m}\right)$.

Demonstração: Consideremos o caso $r=1$. Se $Y_{k} \in \mathcal{H}_{k}\left(S^{m}\right), k \in \mathbb{N}$, usando a Proposicão 2.2.6-(ii) obtemos

$$
\begin{aligned}
\left\|\frac{\Delta_{t}\left(Y_{k}\right)}{1-t}-\frac{k(k+m-1)}{m} Y_{k}\right\|_{X} & =\left\|\frac{\left(1-P_{k}^{m}(t)\right) Y_{k}}{1-t}-\frac{k(k+m-1)}{m} Y_{k}\right\|_{X} \\
& =\left|\frac{1-P_{k}^{m}(t)}{1-t}-\frac{k(k+m-1)}{m}\right|\left\|Y_{k}\right\|_{X}, \quad t \in(-1,1) .
\end{aligned}
$$


Aplicando a Regra de L'Hospital e usando (1.9) vemos que

$$
\lim _{t \rightarrow 1} \frac{1-P_{k}^{m}(t)}{1-t}=\lim _{t \rightarrow 1} \frac{k(k+m-1)}{m} P_{k-1}^{m}(t)=\frac{k(k+m-1)}{m} .
$$

Logo,

$$
\lim _{t \rightarrow 1^{-}}\left\|\frac{\Delta_{t}\left(Y_{k}\right)}{1-t}-\frac{k(k+m-1)}{m} Y_{k}\right\|_{X}=0
$$

Portanto, $Y_{k} \in W_{X}^{1} \mathrm{e}$

$$
D^{1}\left(Y_{k}\right)=\frac{k(k+m-1)}{m} Y_{k} .
$$

Suponhamos que a proposição vale para $r=1,2, \ldots, s-1$. Usando a hipótese de indução com $r=s-1$, segue que

$$
\begin{gathered}
\lim _{t \rightarrow 1^{-}}\left\|\frac{\Delta_{t}\left(D^{s-1}\left(Y_{k}\right)\right)}{1-t}-\left(\frac{k(k+m-1)}{m}\right)^{s} Y_{k}\right\|_{X} \\
=\left(\frac{k(k+m-1)}{m}\right)^{s-1} \lim _{t \rightarrow 1^{-}}\left\|\frac{\Delta_{t}\left(Y_{k}\right)}{1-t}-D^{1}\left(Y_{k}\right)\right\|_{X} .
\end{gathered}
$$

Por outro lado, usando a hípótese de indução com $r=1$, temos que

$$
\lim _{t \rightarrow 1^{-}}\left\|\frac{\Delta_{t}\left(D^{s-1}\left(Y_{k}\right)\right)}{1-t}-\left(\frac{k(k+m-1)}{m}\right)^{s} Y_{k}\right\|_{X}=0 .
$$

Logo, $D^{s-1}\left(Y_{k}\right) \in W_{X}^{1}$. Isto implica que $D^{s}\left(Y_{k}\right)=D^{1}\left(D^{s-1}\left(Y_{k}\right)\right) \in X$, ou seja, que $Y_{k} \in W_{X}^{s}$. Além disso,

$$
D^{s}\left(Y_{k}\right)=\left(\frac{k(k+m-1)}{m}\right)^{s} Y_{k}
$$

Logo, a proposição está provada.

Teorema 2.3.3. Seja r um inteiro positivo. Então o conjunto $W_{X}^{r}$ é denso em $X$.

Demonstração: Isto segue das inclusões $\cup_{k=0}^{\infty} \mathcal{H}_{k}\left(S^{m}\right) \subset W_{X}^{r} \subset X$ e do fato do conjunto $\cup_{k=0}^{\infty} \mathcal{H}_{k}\left(S^{m}\right)$ ser fundamental em $X$.

Proposição 2.3.4. Se r é um inteiro positivo, então o conjunto $W_{X}^{r}$ é um subespaço vetorial de $X$.

Demonstração: Já sabemos que $0 \in W_{X}^{r}$. Para provar a outra condição de subespaço vetorial consideremos primeiramente o caso $r=1$. Sejam $f, g \in W_{X}^{1}$ e $\alpha \in \mathbb{C}$. Usando a linearidade de $\Delta_{t}$ e a desigualdade triangular segue que

$$
\left\|\frac{\Delta_{t}(\alpha f+g)}{1-t}-\left(\alpha D^{1}(f)+D^{1}(g)\right)\right\|_{X} \leq|\alpha|\left\|\frac{\Delta_{t}(f)}{1-t}-D^{1}(f)\right\|_{X}+\left\|\frac{\Delta_{t}(g)}{1-t}-D^{1}(g)\right\|_{X} .
$$


Tomando-se o limite para $t \rightarrow 1^{-}$, concluímos que $D^{1}(\alpha f+g)=\alpha D^{1}(f)+D^{1}(g)$. Como $f, g \in W_{X}^{1}$, segue que $\alpha f+g \in W_{X}^{1}$. Suponhamos que o resultado vale para $r=1,2, \ldots, s-1$ e sejam $f, g \in W_{X}^{s}$ e $\alpha \in \mathbb{R}$. Então, procedendo como acima, temos que

$$
\begin{array}{r}
\left\|\frac{\Delta_{t}\left(D^{s-1}(\alpha f+g)\right)}{1-t}-\left(\alpha D^{s}(f)+D^{s}(g)\right)\right\|_{X} \leq|\alpha|\left\|\frac{\Delta_{t}\left(D^{s-1}(f)\right)}{1-t}-D^{s}(f)\right\|_{X} \\
+\left\|\frac{\Delta_{t}\left(D^{s-1}(g)\right)}{1-t}-D^{s}(g)\right\|_{X} .
\end{array}
$$

Tomando-se o limite na desigualdade acima para $t \rightarrow 1^{-}$vem que

$$
\lim _{t \rightarrow 1^{-}}\left\|\frac{\Delta_{t}\left(D^{s-1}(\alpha f+g)\right)}{1-t}-\left(\alpha D^{s}(f)+D^{s}(g)\right)\right\|_{X}=0 .
$$

Logo, $D^{s}(\alpha f+g)=\alpha D^{s}(f)+D^{s}(g)$. Como $f, g \in W_{X}^{s}$, segue que $\alpha f+g \in W_{X}^{s}$.

Corolário 2.3.5. Para cada inteiro positivo $r$, a derivada forte de Laplace-Beltrami $D^{r}$ é linear.

Uma maneira natural de gerar uma topologia para o espaço $W_{X}^{r}$ é considerar a norma $\|\cdot\|_{W_{X}^{r}}$ dada por

$$
\|f\|_{W_{X}^{r}}=\|f\|_{X}+\left\|D^{r}(f)\right\|_{X}, \quad f \in W_{X}^{r} .
$$

Mostraremos no final da Seção 2.7 que o espaço $\left(W_{X}^{r},\|\cdot\|_{W_{X}^{r}}\right)$ é completo.

A ação do operador projeção sobre a derivada forte de Laplace-Beltrami é explicada abaixo.

Teorema 2.3.6. Sejam $r$ um inteiro positivo e $f \in W_{X}^{r}$. Então

$$
\mathcal{Y}_{n}\left(D^{r}(f)\right)=\left(\frac{n(n+m-1)}{m}\right)^{r} \mathcal{Y}_{n}(f), \quad n \in \mathbb{N} .
$$

Demonstração: Fixe $n \in \mathbb{N}$. No caso $r=1$, usamos inicialmente a fórmula (1.9) para deduzir que, para $x \in S^{m}$ e $f \in W_{X}^{1}$,

$$
\begin{aligned}
\left|\frac{n(n+m-1)}{m} \mathcal{Y}_{n}(f)(x)-\mathcal{Y}_{n}\left(D^{1}(f)\right)(x)\right| & =\left|\frac{d}{d t} P_{n}^{m}(t) \mathcal{Y}_{n}(f)(x)-\mathcal{Y}_{n}\left(D^{1}(f)\right)(x)\right| \\
& =\lim _{t \rightarrow 1^{-}}\left|\frac{1-P_{n}^{m}(t)}{1-t} \mathcal{Y}_{n}(f)(x)-\mathcal{Y}_{n}\left(D^{1}(f)\right)(x)\right|
\end{aligned}
$$

Pela Proposição 2.2.7 e pela linearidade de $\mathcal{Y}_{n}$, podemos escrever

$$
\begin{aligned}
\left|\frac{1-P_{n}^{m}(t)}{1-t} \mathcal{Y}_{n}(f)(x)-\mathcal{Y}_{n}\left(D^{1}(f)\right)(x)\right| & =\left|\frac{\mathcal{Y}_{n}(f)(x)-\mathcal{Y}_{n}\left(S_{t}^{m}(f)\right)(x)}{1-t}-\mathcal{Y}_{n}\left(D^{1}(f)\right)(x)\right| \\
& =\left|\mathcal{Y}_{n}\left(\frac{f-S_{t}^{m}(f)}{1-t}-D^{1}(f)\right)(x)\right| .
\end{aligned}
$$

Por outro lado, a Proposição 2.1.3-(ii) implica que

$$
\lim _{t \rightarrow 1^{-}}\left|\mathcal{Y}_{n}\left(\frac{f-S_{t}^{m}(f)}{1-t}-D^{1}(f)\right)(x)\right| \leq N(m, n) \lim _{t \rightarrow 1^{-}}\left\|\frac{f-S_{t}^{m}(f)}{1-t}-D^{1}(f)\right\|_{X}=0 .
$$


Combinando-se as três informações anteriores, concluímos que

$$
\mathcal{Y}_{n}\left(D^{1}(f)\right)=\frac{n(n+m-1)}{m} \mathcal{Y}_{n}(f) .
$$

Suponhamos que o resultado vale para $r=1,2, \ldots, s-1$. Então

$$
\begin{aligned}
\mathcal{Y}_{n}\left(D^{s}(f)\right) & =\mathcal{Y}_{n}\left(D^{1}\left(D^{s-1}(f)\right)\right) \\
& =\left(\frac{n(n+m-1)}{m}\right) \mathcal{Y}_{n}\left(D^{s-1}(f)\right) \\
& =\left(\frac{n(n+m-1)}{m}\right)^{s} \mathcal{Y}_{n}(f), \quad f \in W_{X}^{s} .
\end{aligned}
$$

Assim, o teorema segue.

Como a imagem de $\mathcal{Y}_{n}$ é $\mathcal{H}_{n}\left(S^{m}\right)$, combinando-se a Proposição 2.3.2-(ii) e o teorema anterior, deduzimos o seguinte corolário.

Corolário 2.3.7. Se $r$ é um inteiro positivo e n é um inteiro não-negativo, então $\mathcal{Y}_{n} \circ D^{r}=$ $D^{r} \circ \mathcal{Y}_{n}$ em $W_{X}^{r}$.

Proposição 2.3.8. Sejam $r$ um inteiro positivo e $f \in X$. As seguintes afirmações são equivalentes:

(i) $f \in W_{X}^{r}$ e $D^{r}(f)=0$;

(ii) $f$ é constante.

Demonstração: Se $f$ é contante, $\Delta_{t}(f)=0, t \in(-1,1)$. Consequentemente, $f \in W_{X}^{1}$ e $D^{1}(f)=0$. Agora está claro que $D^{1}(f) \in W_{X}^{r-1}$ e que $D^{r-1}\left(D^{1}(f)\right)=0$, quando $r \geq 2$. Logo, $f \in W_{X}^{r}$ e $D^{r}(f)=0$. Reciprocamente, suponha que $f \in W_{X}^{r}$ e $D^{r}(f)=0$. Do Teorema 2.3.6, segue que

$$
0=\mathcal{Y}_{n}\left(D^{r}(f)\right)=\left(\frac{n(n+m-1)}{m}\right)^{r} \mathcal{Y}_{n}(f), \quad n \in \mathbb{N} .
$$

Portanto, $\mathcal{Y}_{n}(f)=0, n=1,2, \ldots$ Procedendo-se como na prova do Teorema 2.1.2, concluímos que $\hat{f}(k, l)=0, k=1,2, \ldots, l=1,2, \ldots, N(m, k)$. Logo, $f-\hat{f}(0,1) Y_{01}=0$, o que implica que $f$ é constante.

O próximo resultado descreve a ação da derivada forte de Laplace-Beltrami sobre a translação esférica.

Proposição 2.3.9. Sejam $r$ um inteiro positivo, $f \in W_{X}^{r}$ et $t \in(-1,1)$. Então $S_{t}^{m}(f) \in W_{X}^{r}$ e $D^{r}\left(S_{t}^{m}(f)\right)=S_{t}^{m}\left(D^{r}(f)\right)$.

Demonstração: É suficiente provar que $S_{t}^{m}(f) \in W_{X}^{1}$ e que $D^{1}\left(S_{t}^{m}(f)\right)=S_{t}^{m}\left(D^{1}(f)\right)$, quando $f \in W_{X}^{1}$. Se $f \in W_{X}^{1}$, usamos a Proposição 2.2.9 e a linearidade de $S_{t}^{m}$ para obter

$$
\Delta_{h}\left(S_{t}^{m}(f)\right)=S_{t}^{m}(f)-S_{h}^{m}\left(S_{t}^{m}(f)\right)=S_{t}^{m}(f)-S_{t}^{m}\left(S_{h}^{m}(f)\right)=S_{t}^{m}\left(\Delta_{h}(f)\right), \quad h \in(-1,1) .
$$


Logo,

$$
\begin{aligned}
\frac{\Delta_{h}\left(S_{t}^{m}(f)\right)}{1-h}-S_{t}^{m}\left(D^{1}(f)\right) & =\frac{S_{t}^{m}\left(\Delta_{h}(f)\right)}{1-h}-S_{t}^{m}\left(D^{1}(f)\right) \\
& =S_{t}^{m}\left(\frac{\Delta_{h}(f)}{1-h}-D^{1}(f)\right), \quad h \in(-1,1) .
\end{aligned}
$$

Como $f \in W_{X}^{1}$, segue que

$$
\lim _{h \rightarrow 1^{-}}\left\|\frac{\Delta_{h}\left(S_{t}^{m}(f)\right)}{1-h}-S_{t}^{m}\left(D^{1}(f)\right)\right\|_{X} \leq\left\|S_{t}^{m}\right\|_{X} \lim _{h \rightarrow 1^{-}}\left\|\frac{\Delta_{h}(f)}{1-h}-D^{1}(f)\right\|_{X}=0 .
$$

Portanto,

$$
\lim _{h \rightarrow 1^{-}}\left\|\frac{\Delta_{h}\left(S_{t}^{m}(f)\right)}{1-h}-S_{t}^{m}\left(D^{1}(f)\right)\right\|_{X}=0 .
$$

Assim, $S_{t}^{m}(f) \in W_{X}^{1}$ e $D^{1}\left(S_{t}^{m}(f)\right)=S_{t}^{m}\left(D^{1}(f)\right)$.

Corolário 2.3.10. Se r é um inteiro positivo, então $D^{r} \circ \Delta_{t}=\Delta_{t} \circ D^{r}$ em $W_{X}^{r}$.

Corolário 2.3.11. Sejam $r, s$ inteiros positivos e $t_{1}, t_{2}, \ldots, t_{s} \in(-1,1)$. Então,

$$
\Delta_{t_{1}} \circ \Delta_{t_{2}} \circ \ldots \circ \Delta_{t_{s}} \circ D^{r}=D^{r} \circ \Delta_{t_{1}} \circ \Delta_{t_{2}} \circ \ldots \circ \Delta_{t_{s}}
$$

em $W_{X}^{r}$.

\subsection{A integral de Laplace-Beltrami}

Nesta seção, descreveremos um operador que atua como um operador inverso para $D^{r}$, pelo menos quando ambos estão atuando nos elementos de $\left[\cup_{k=1}^{\infty} \mathcal{H}_{k}\left(S^{m}\right)\right]$. Construções semelhantes foram consideradas por Rudin ([28]) e Wehrens ([37]), no caso de $S^{2}$.

Levando-se em conta a Proposição 2.3.2, a idéia aqui é construir um operador $J^{r}$ tal que

$$
J^{r}\left(Y_{k}\right)=\left(\frac{m}{k(k+m-1)}\right)^{r} Y_{k}, \quad Y_{k} \in \mathcal{H}_{k}\left(S^{m}\right), \quad k=1,2, \ldots
$$

O passo principal é considerar a função $\mathfrak{L}$ dada abaixo.

Lema 2.4.1. Seja $\mathfrak{L}:(-1,1) \rightarrow \mathbb{R}$ a função definida por

$$
\mathfrak{L}(t)=m \int_{-1}^{t}\left(1-s^{2}\right)^{-m / 2} \int_{-1}^{s} d w_{m}(u) d s .
$$

Então $\mathfrak{L} \in L^{1, m}\left([-1,1], d w_{m}\right) e$

$$
\mathfrak{L}^{\wedge}(n)= \begin{cases}\frac{m}{n(n+m-1)}, & n=1,2, \ldots \\ \|\mathfrak{L}\|_{1, m}, & n=0 .\end{cases}
$$


Demonstração: Para provar que $\mathfrak{L} \in L^{1, m}\left([-1,1], d w_{m}\right)$, analisaremos dois casos separados.

No caso $m=2$,

$$
\mathfrak{L}(t)=2 \int_{-1}^{t}\left(1-s^{2}\right)^{-1} \int_{-1}^{s} d u d s=2 \ln \frac{2}{1-t}
$$

Logo,

$$
\frac{\sigma_{1}}{\sigma_{2}} \int_{-1}^{1} \mathfrak{L}(t) d t=\int_{-1}^{1} \ln \frac{2}{1-t} d t=2,
$$

o que implica que $\mathfrak{L} \in L^{1,2}(-1,1)$. Como $\mathfrak{L}(t) \geq 0, t \in(-1,1)$, segue que

$$
\mathfrak{L} \Upsilon(0)=\|\mathfrak{L}\|_{1,2}=\frac{\sigma_{1}}{\sigma_{2}} \int_{-1}^{1} \mathfrak{L}(t) d t=2 .
$$

No caso $m>2$, usando a Regra de L'Hospital ([29], p. 109), temos que

$$
\begin{aligned}
\lim _{t \rightarrow-1^{+}} \mathfrak{L}(t)\left(1-t^{2}\right)^{(m-2) / 2} & =\lim _{t \rightarrow-1^{+}} \frac{m \int_{-1}^{t}\left(1-s^{2}\right)^{-m / 2} \int_{-1}^{s} d w_{m}(u) d s}{\left(1-t^{2}\right)^{-(m-2) / 2}} \\
& =\lim _{t \rightarrow-1^{+}} \frac{m}{t(m-2)} \int_{-1}^{t} d w_{m}(u) \\
& =0 .
\end{aligned}
$$

De maneira análoga, podemos mostrar que

$$
\lim _{t \rightarrow 1^{-}} \mathfrak{L}(t)\left(1-t^{2}\right)^{(m-2) / 2}=\frac{m}{m-2} \int_{-1}^{1} d w_{m}(u)<\infty .
$$

Logo, como $\mathfrak{L}$ é contínua em $(-1,1)$, segue que $\mathfrak{L} \in L^{1, m}\left([-1,1], d w_{m}\right)$. Para concluir a prova, primeiramente observamos que a não-negatividade de $\mathfrak{L}$ implica que

$$
\mathfrak{L}-(0)=\frac{\sigma_{m-1}}{\sigma_{m}} \int_{-1}^{1} \mathfrak{L}(t) d w_{m}(t)=\|\mathfrak{L}\|_{1, m} .
$$

Para calcular $\mathfrak{L}^{\Upsilon}(n), n \geq 1$, primeiro escrevemos

$$
\begin{aligned}
\mathfrak{L}^{-}(n) & =\frac{\sigma_{m-1}}{\sigma_{m}} \int_{-1}^{1} \mathfrak{L}(t) P_{n}^{m}(t) d w_{m}(t) \\
& =\frac{m \sigma_{m-1}}{C_{n}^{(m-1) / 2}(1) \sigma_{m}} \int_{-1}^{1} \int_{-1}^{t}\left(1-s^{2}\right)^{-m / 2} \int_{-1}^{s} d w_{m}(u) d s C_{n}^{(m-1) / 2}(t) d w_{m}(t)
\end{aligned}
$$

Usando integração por partes e a Proposição 1.2.2 vemos que

$$
\begin{aligned}
\mathfrak{L}^{\Upsilon}(n)=- & D(m) \lim _{t \rightarrow 1^{-}} \int_{-1}^{t}\left(1-s^{2}\right)^{-m / 2} \int_{-1}^{s} d w_{m}(u) d s\left(1-t^{2}\right)^{m / 2} C_{n-1}^{(m+1) / 2}(t) \\
& +D(m) \lim _{t \rightarrow-1^{+}} \int_{-1}^{t}\left(1-s^{2}\right)^{-m / 2} \int_{-1}^{s} d w_{m}(u) d s\left(1-t^{2}\right)^{m / 2} C_{n-1}^{(m+1) / 2}(t) \\
& +D(m) \int_{-1}^{1} \int_{-1}^{t} d w_{m}(u) C_{n-1}^{(m+1) / 2}(t) d t
\end{aligned}
$$


onde

$$
D(m):=\frac{\sigma_{m-1}}{C_{n}^{(m-1) / 2}(1) \sigma_{m}} \frac{m(m-1)}{n(n+m-1)} .
$$

Usando o que foi feito na parte anterior, temos que

$$
\begin{gathered}
\lim _{t \rightarrow 1^{-}} \int_{-1}^{t}\left(1-s^{2}\right)^{-m / 2} \int_{-1}^{s} d w_{m}(u) d s\left(1-t^{2}\right)^{m / 2} C_{n-1}^{(m+1) / 2}(t)= \\
\lim _{t \rightarrow 1^{-}} \int_{-1}^{t}\left(1-s^{2}\right)^{-m / 2} \int_{-1}^{s} d w_{m}(u) d s\left(1-t^{2}\right)^{(m-2) / 2}\left(1-t^{2}\right) C_{n-1}^{(m+1) / 2}(t)=0 .
\end{gathered}
$$

Analogamente, mostramos que

$$
\lim _{t \rightarrow-1^{+}} \int_{-1}^{t}\left(1-s^{2}\right)^{-m / 2} \int_{-1}^{s} d w_{m}(u) d s\left(1-t^{2}\right)^{m / 2} C_{n-1}^{(m+1) / 2}(t)=0 .
$$

Logo, integrando por partes novamente, obtemos

$$
\begin{aligned}
\mathfrak{L}^{-}(n) & =D(m) \int_{-1}^{1} \int_{-1}^{t} d w_{m}(u) C_{n-1}^{(m+1) / 2}(t) d t \\
& =\frac{D(m)}{m-1}\left[\left.C_{n}^{(m-1) / 2}(t) \int_{-1}^{t} d w_{m}(u)\right|_{-1} ^{1}-\int_{-1}^{1} C_{n}^{(m-1) / 2}(t) d w_{m}(t)\right] \\
& =\frac{D(m)}{m-1}\left[C_{n}^{(m-1) / 2}(1) \int_{-1}^{1} d w_{m}(u)-\int_{-1}^{1} C_{n}^{(m-1) / 2}(t) d w_{m}(t)\right] .
\end{aligned}
$$

A Proposição 1.2.2 garante que

$$
\int_{-1}^{1} C_{n}^{(m-1) / 2}(t) d w_{m}(t)=-\left.\frac{m-1}{n(n+m-1)}\left(1-t^{2}\right)^{m / 2} C_{n-1}^{(m+1) / 2}(t)\right|_{-1} ^{1}=0
$$

Logo, usando a Fórmula de Funk-Hecke, segue que

$$
\begin{aligned}
\mathfrak{L}^{\Upsilon}(n) & =\frac{D(m)}{m-1} C_{n}^{(m-1) / 2}(1) \int_{-1}^{1} d w_{m}(u) \\
& =\frac{m}{n(n+m-1)} \frac{\sigma_{m-1}}{\sigma_{m}} \int_{-1}^{1} d w_{m}(u) \\
& =\frac{m}{n(n+m-1)} .
\end{aligned}
$$

Isso completa a prova do lema.

No próximo resultado, normalizamos convenientemente a função $\mathfrak{L}$ introduzida acima, de forma que seu primeiro coeficiente de Fourier-Legendre seja igual a 1.

Teorema 2.4.2. Seja $\mathcal{L}:(-1,1) \rightarrow \mathbb{R}$ a função definida por

$$
\mathcal{L}(t)=\mathfrak{L}(t)+1-\|\mathfrak{L}\|_{1, m} .
$$

Então $\mathcal{L} \in L^{1, m}\left([-1,1], d w_{m}\right) e$

$$
\mathcal{L}^{\Upsilon}(n)= \begin{cases}\frac{m}{n(n+m-1)}, & n=1,2, \ldots \\ 1, & n=0 .\end{cases}
$$


Demonstração: Como $\mathfrak{L} \in L^{1, m}\left([-1,1], d w_{m}\right)$ segue que $\mathcal{L} \in L^{1, m}\left([-1,1], d w_{m}\right)$. Usando a Fórmula de Funk-Hecke, obtemos

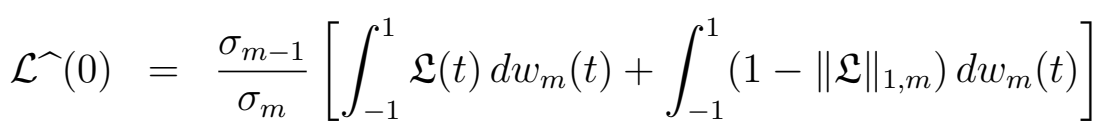

$$
\begin{aligned}
& =\|\mathfrak{L}\|_{1, m}+\left(1-\|\mathfrak{L}\|_{1, m}\right) \frac{\sigma_{m-1}}{\sigma_{m}} \int_{-1}^{1} d w_{m}(t) \\
& =\|\mathfrak{L}\|_{1, m}+\left(1-\|\mathfrak{L}\|_{1, m}\right) \frac{1}{\sigma_{m}} \int_{S^{m}} d \sigma_{m} \\
& =1 \text {. }
\end{aligned}
$$

Se $n \geq 1$, a ortogonalidade de $\left\{P_{k}^{m}: k=0,1, \ldots\right\}$ em $L^{1, m}\left([-1,1], d w_{m}\right)$ e o lema anterior, implicam que

$$
\begin{aligned}
\mathcal{L}^{\curlywedge}(n) & =\frac{\sigma_{m-1}}{\sigma_{m}}\left[\int_{-1}^{1} \mathfrak{L}(t) P_{n}^{m}(t) d w_{m}(t)+\left(1-\|\mathfrak{L}\|_{1, m}\right) \int_{-1}^{1} P_{n}^{m}(t) d w_{m}(t)\right] \\
& =\mathfrak{L}^{\curlyvee}(n)+\left(1-\|\mathfrak{L}\|_{1, m}\right) \frac{\sigma_{m-1}}{\sigma_{m}} \int_{-1}^{1} P_{n}^{m}(t) P_{0}^{m}(t) d w_{m}(t) \\
& =\mathfrak{L}^{\mathfrak{l}}(n) .
\end{aligned}
$$

Logo, a prova está completa.

A seguir, introduziremos o operador integral de Laplace-Beltrami por meio de uma convolução com a função $\mathcal{L}$.

Definição 2.4.3. Seja $r$ um inteiro positivo. O operador integral de Laplace-Beltrami é o operador $J^{r}: X \rightarrow X$ dado por

$$
J^{1}(f):=\mathcal{L} * f, \quad f \in X
$$

e, indutivamente, por

$$
J^{r}(f):=J^{1}\left(J^{r-1}(f)\right), \quad r=2,3, \ldots, \quad f \in X .
$$

Notemos que a Proposição 1.2 .5 garante que o operador $J^{r}$ está bem definido e que $\left\|J^{r}\right\|_{X} \leq\|\mathcal{L}\|_{1, m}^{r}$

Teorema 2.4.4. Seja r um inteiro positivo. Então vale a seguinte propriedade:

$$
\mathcal{Y}_{n} \circ J^{r}= \begin{cases}\left(\frac{m}{n(n+m-1)}\right)^{r} \mathcal{Y}_{n}, & n=1,2, \ldots, \\ \mathcal{Y}_{0}, & n=0 .\end{cases}
$$


Demonstração: Usando a Proposição 1.2 .5 e o Teorema 2.4.2, temos que

$$
\begin{aligned}
& \mathcal{Y}_{n} \circ J^{r}(f)=\mathcal{Y}_{n}\left(\left(\mathcal{L} * J^{r-1}\right)(f)\right) \\
& =\mathcal{L}^{\mathfrak{1}}(n) \mathcal{Y}_{n}\left(J^{r-1}(f)\right) \\
& \vdots \\
& =\mathcal{L}^{\widehat{\gamma}}(n)^{r} \mathcal{Y}_{n}(f) \\
& =\left(\frac{m}{n(n+m-1)}\right)^{r} \mathcal{Y}_{n}(f), \quad n=1,2, \ldots, \quad f \in X .
\end{aligned}
$$

Se $n=0$,

$$
\mathcal{Y}_{0} \circ J^{r}(f)=\mathcal{L}^{\Upsilon}(0)^{r} \mathcal{Y}_{0}(f)=\mathcal{Y}_{0}(f), \quad f \in X .
$$

Logo, o teorema está provado.

Teorema 2.4.5. Seja $r$ um inteiro positivo. Então $J^{r} \circ D^{r}=I-\mathcal{Y}_{0}$ em $W_{X}^{r}$. Em particular, $W_{X}^{r}$ é $J^{r} \circ D^{r}$-invariante.

Demonstração: Seja $f \in W_{X}^{r}$. Usando o teorema anterior e o Teorema 2.3.6, segue que

$$
\mathcal{Y}_{n}\left(J^{r}\left(D^{r}(f)\right)\right)=\left(\frac{m}{n(n+m-1)}\right)^{r} \mathcal{Y}_{n}\left(D^{r}(f)\right)=\mathcal{Y}_{n}(f), \quad n=1,2, \ldots,
$$

ou seja, $J^{r}\left(D^{r}(f)\right)-f$ é uma constante em $X$. Em particular, $J^{r}\left(D^{r}(f)\right) \in W_{X}^{r}$. Finalmente, dos Teoremas 2.4 .4 e 2.3 .6 e do fato de que $\mathcal{Y}_{0}$ é uma projeção, temos que

$$
\mathcal{Y}_{0}\left(J^{r}\left(D^{r}(f)\right)-f\right)=\mathcal{Y}_{0}\left(D^{r}(f)\right)-\mathcal{Y}_{0}(f)=-\mathcal{Y}_{0}(f)
$$

Dessa forma, a constante é $-\mathcal{Y}_{0}(f)$.

O corolário seguinte é uma conseqüência das propriedades mencionadas acima e das Proposições 2.1.3 e 2.3.2.

Corolário 2.4.6. Valem as seguintes afirmações:

(i) A restrição de $J^{r} \circ D^{r} a\left[\cup_{k=1}^{\infty} \mathcal{H}_{k}\left(S^{m}\right)\right]$ é o operador identidade;

(ii) Para $k=1,2, \ldots$,

$$
J^{r}\left(Y_{k}\right)=\left(\frac{m}{k(k+m-1)}\right)^{r} Y_{k}, \quad Y_{k} \in \mathcal{H}_{k}\left(S^{m}\right) .
$$

Demonstração: Seja $Y_{k} \in \mathcal{H}_{k}\left(S^{m}\right), k=1,2, \ldots$ A Proposição 2.1.3- $(i)$ garante que $\mathcal{Y}_{0}\left(Y_{k}\right)=0$. Logo, usando o teorema anterior, segue que

$$
J^{r}\left(D^{r}\left(Y_{k}\right)\right)=Y_{k} .
$$

Isso prova a parte $(i)$. Usando o item anterior, o Teorema 2.3.6 e a linearidade de $J^{r}$ vemos que

$$
Y_{k}=J^{r}\left(D^{r}\left(Y_{k}\right)\right)=\left(\frac{k(k+m-1)}{m}\right)^{r} J^{r}\left(Y_{k}\right)
$$


Logo,

$$
J^{r}\left(Y_{k}\right)=\left(\frac{m}{k(k+m-1)}\right)^{r} Y_{k}
$$

Isso conclui a prova.

\subsection{A função $\mathcal{L}_{h}$}

Nesta seção, definiremos uma função $\mathcal{L}_{h} \in L^{1, m}\left([-1,1], d w_{m}\right)$ que satisfaz

$$
\mathcal{L}_{h} \widehat{(}(n)=\frac{1-P_{n}^{m}(h)}{n(n+m-1)}, \quad n=1,2, \ldots
$$

Em vista da Proposição 2.2.8 e do Teorema 2.4.4, o efeito da convolução dessa função com elementos de $X$ deve ser similar àquele da ação do operador $\Delta_{h} \circ J^{1}$ nesses elementos.

Lema 2.5.1. Seja $n$ um inteiro não-negativo. A função $I_{n}^{m}:(-1,1) \rightarrow \mathbb{R}$ definida por

$$
I_{n}^{m}(h):=\sigma_{m-1} \int_{h}^{1} P_{n}^{m}(t) d w_{m}(t) .
$$

satisfaz

$$
\frac{1}{\sigma_{m-1}} \int_{h}^{1} I_{n}^{m}(u)\left(1-u^{2}\right)^{-m / 2} d u=\frac{1-P_{n}^{m}(h)}{n(n+m-1)} .
$$

Demonstração: Substituindo a expressão de $I_{n}^{m}$ na integral em (2.9) obtemos

$$
\begin{aligned}
\int_{h}^{1} I_{n}^{m}(u)\left(1-u^{2}\right)^{-m / 2} d u & =\sigma_{m-1} \int_{h}^{1}\left(\int_{u}^{1} P_{n}^{m}(t) d w_{m}(t)\right)\left(1-u^{2}\right)^{-m / 2} d u \\
& =\frac{\sigma_{m-1}}{C_{n}^{(m-1) / 2}(1)} \int_{h}^{1}\left(\int_{u}^{1} C_{n}^{(m-1) / 2}(t) d w_{m}(t)\right)\left(1-u^{2}\right)^{-m / 2} d u
\end{aligned}
$$

Mas, a Proposição 1.2.2 garante que

$$
\int_{u}^{1} C_{n}^{(m-1) / 2}(t) d w_{m}(t)=\frac{m-1}{n(n+m-1)}\left(1-u^{2}\right)^{m / 2} C_{n-1}^{(m+1) / 2}(u) .
$$

Logo,

$$
\frac{1}{\sigma_{m-1}} \int_{h}^{1} I_{n}^{m}(u)\left(1-u^{2}\right)^{-m / 2} d u=\frac{m-1}{n(n+m-1) C_{n}^{(m-1) / 2}(1)} \int_{h}^{1} C_{n-1}^{(m+1) / 2}(u) d u
$$

Como

$$
\int_{h}^{1} C_{n-1}^{(m+1) / 2}(u) d u=\frac{1}{m-1}\left(C_{n}^{(m-1) / 2}(1)-C_{n}^{(m-1) / 2}(h)\right),
$$

o uso da Fórmula (1.9) nos leva a concluir que

$$
\begin{aligned}
\frac{1}{\sigma_{m-1}} \int_{h}^{1} I_{n}^{m}(u)\left(1-u^{2}\right)^{-m / 2} d u & =\frac{C_{n}^{(m-1) / 2}(1)-C_{n}^{(m-1) / 2}(h)}{n(n+m-1) C_{n}^{(m-1) / 2}(1)} \\
& =\frac{1-P_{n}^{m}(h)}{n(n+m-1)}
\end{aligned}
$$


Isso completa a prova do lema.

Usando o lema acima, vemos facilmente que a função $\mathcal{L}_{h}$ que procuramos deve satisfazer

$$
\begin{aligned}
\frac{\sigma_{m-1}}{\sigma_{m}} \int_{-1}^{1} \mathcal{L}_{h}(t) P_{n}^{m}(t) d w_{m}(t) & =\frac{1}{\sigma_{m-1}} \int_{h}^{1} I_{n}^{m}(u)\left(1-u^{2}\right)^{-m / 2} d u \\
& =\int_{h}^{1}\left(\int_{u}^{1} P_{n}^{m}(t) d w_{m}(t)\right)\left(1-u^{2}\right)^{-m / 2} d u
\end{aligned}
$$

Uma mudança de variáveis reduz essa igualdade a

$$
\begin{aligned}
\frac{\sigma_{m-1}}{\sigma_{m}} \int_{-1}^{1} \mathcal{L}_{h}(t) P_{n}^{m}(t) d w_{m}(t) & =\int_{h}^{1}\left(\int_{h}^{t}\left(1-u^{2}\right)^{-m / 2} d u\right) P_{n}^{m}(t) d w_{m}(t) \\
& =\int_{-1}^{1} \chi_{[h, 1)}(t)\left(\int_{h}^{t}\left(1-u^{2}\right)^{-m / 2} d u\right) P_{n}^{m}(t) d w_{m}(t) .
\end{aligned}
$$

Na igualdade acima, o símbolo $\chi_{A}$ denota a função característica do conjunto $A$.

Lema 2.5.2. Sejam $h \in(-1,1)$ e $\mathcal{L}_{h}$ a função definida por

$$
\mathcal{L}_{h}(t):=\frac{\sigma_{m}}{\sigma_{m-1}} \chi_{[h, 1)}(t) \int_{h}^{t}\left(1-u^{2}\right)^{-m / 2} d u, \quad t \in(-1,1) .
$$

Então $\mathcal{L}_{h} \in L^{1, m}\left([-1,1], d w_{m}\right) e$

$$
\mathcal{L}_{h} \widehat{(}(n)= \begin{cases}\frac{1-P_{n}^{m}(h)}{n(n+m-1)}, & n=1,2, \ldots \\ \left\|\mathcal{L}_{h}\right\|_{1, m}, & n=0\end{cases}
$$

Demonstração: Observemos primeiramente que $\mathcal{L}_{h}$ é não-negativa. Para mostrar que $\mathcal{L}_{h} \in$ $L^{1, m}\left([-1,1], d w_{m}\right)$, analisaremos dois casos. Se $m=2$, então

$$
\begin{aligned}
\frac{1}{2} \int_{-1}^{1} \mathcal{L}_{h}(t) d w_{2}(t) & =\int_{h}^{1}\left(\int_{h}^{t}\left(1-u^{2}\right)^{-1} d u\right) d t \\
& =\frac{1}{2} \int_{h}^{1} \ln \frac{(1+t)(1-h)}{(1-t)(1+h)} d t \\
& =\ln \frac{2}{1+h}<\infty .
\end{aligned}
$$

Se $m \geq 3$, aplicando a Regra de L'Hospital, segue que

$$
\begin{aligned}
\lim _{t \rightarrow 1}\left(1-t^{2}\right)^{(m-2) / 2} \int_{h}^{t}\left(1-u^{2}\right)^{-m / 2} d u & =\lim _{t \rightarrow 1} \frac{\int_{h}^{t}\left(1-u^{2}\right)^{-m / 2} d u}{\left(1-t^{2}\right)^{-(m-2) / 2}} \\
& =\lim _{t \rightarrow 1} \frac{1}{(m-2) t} \\
& =\frac{1}{m-2}<\infty .
\end{aligned}
$$

Como a função

$$
t \in[h, 1) \mapsto\left(1-t^{2}\right)^{(m-2) / 2} \int_{h}^{t}\left(1-u^{2}\right)^{-m / 2} d u
$$


é contínua, temos que

$$
\frac{\sigma_{m-1}}{\sigma_{m}} \int_{h}^{1}\left(\int_{h}^{t}\left(1-u^{2}\right)^{-m / 2} d u\right) d w_{m}(t)<\infty .
$$

Portanto, $\mathcal{L}_{h} \in L^{1, m}\left([-1,1], d w_{m}\right)$. A fórmula na afirmação do lema, segue trivialmente no caso $n=0$, já que $\mathcal{L}_{h}$ é não-negativa. Se $n \geq 1$, então

$$
\begin{aligned}
\mathcal{L}_{h} \widehat{ }(n) & =\frac{\sigma_{m-1}}{\sigma_{m}} \int_{-1}^{1} L_{h}(t) P_{n}^{m}(t) d w_{m}(t) \\
& =\frac{1}{C_{n}^{(m-1) / 2}(1)} \int_{h}^{1}\left(\int_{h}^{t}\left(1-u^{2}\right)^{-m / 2} d u\right) C_{n}^{(m-1) / 2}(t) d w_{m}(t) \\
& =\frac{1}{C_{n}^{(m-1) / 2}(1)} \lim _{\epsilon \rightarrow 1^{-}} \int_{h}^{\epsilon}\left(\int_{h}^{t}\left(1-u^{2}\right)^{-m / 2} d u\right) C_{n}^{(m-1) / 2}(t) d w_{m}(t) .
\end{aligned}
$$

Se $l$ é o limite acima, integração por partes nos leva a

$$
\begin{aligned}
& l=\frac{m-1}{n(n+m-1)} \lim _{\epsilon \rightarrow 1^{-}}\left[-\left.\int_{h}^{t}\left(1-u^{2}\right)^{-m / 2} d u\left(1-t^{2}\right)^{m / 2} C_{n-1}^{(m+1) / 2}(t)\right|_{h} ^{\epsilon}\right. \\
&\left.+\int_{h}^{\epsilon} C_{n-1}^{(m+1) / 2}(t) d t\right] \\
&=\frac{m-1}{n(n+m-1)}\left[-\lim _{\epsilon \rightarrow 1^{-}} \int_{h}^{\epsilon}\left(1-u^{2}\right)^{-m / 2} d u\left(1-\epsilon^{2}\right)^{m / 2} C_{n-1}^{(m+1) / 2}(\epsilon)\right.\left.+\int_{h}^{1} C_{n-1}^{(m+1) / 2}(t) d t\right] .
\end{aligned}
$$

Mas, usando a regra de L'Hospital uma vez mais, temos que

$$
\begin{aligned}
\lim _{\epsilon \rightarrow 1^{-}}\left(1-\epsilon^{2}\right)^{m / 2} \int_{h}^{\epsilon}\left(1-u^{2}\right)^{-m / 2} d u & =\lim _{\epsilon \rightarrow 1^{-}} \frac{\int_{h}^{\epsilon}\left(1-u^{2}\right)^{-m / 2} d u}{\left(1-\epsilon^{2}\right)^{-m / 2}} \\
& =\lim _{\epsilon \rightarrow 1^{-}} \frac{\left(1-\epsilon^{2}\right)^{-m / 2}}{m\left(1-\epsilon^{2}\right)^{-(m+2) / 2} \epsilon} \\
& =\lim _{\epsilon \rightarrow 1^{-}} \frac{\left(1-\epsilon^{2}\right)}{m \epsilon} \\
& =0 .
\end{aligned}
$$

Logo,

$$
\lim _{\epsilon \rightarrow 1^{-}} \int_{h}^{\epsilon}\left(1-u^{2}\right)^{-m / 2} d u\left(1-\epsilon^{2}\right)^{m / 2} C_{n-1}^{(m+1) / 2}(\epsilon)=0 .
$$

Assim, usando (1.9),

$$
\begin{aligned}
\mathcal{L}_{h} \widehat{(}(n) & =\frac{m}{n(n+m-1) C_{n}^{(m-1) / 2}(1)} \int_{h}^{1} C_{n-1}^{(m+1) / 2}(t) d t \\
& =\frac{1}{n(n+m-1) C_{n}^{(m-1) / 2}(1)}\left[C_{n}^{(m-1) / 2}(1)-C_{n}^{(m-1) / 2}(h)\right] \\
& =\frac{1-P_{n}^{m}(h)}{n(n+m-1)},
\end{aligned}
$$

o que completa a verificação da fórmula. 
Lema 2.5.3. Sejam $h \in(-1,1)$ e $\mathcal{L}_{h}$ a função definida no lema anterior. Defina

$$
G_{h}(t):=\left\|\mathcal{L}_{h}\right\|_{1, m}^{-1} \mathcal{L}_{h}(t), \quad t \in(-1,1) .
$$

Então $G_{h} \in L^{1, m}\left([-1,1], d w_{m}\right) e$

$$
G_{h} \widehat{(}(n)= \begin{cases}\frac{\left\|\mathcal{L}_{h}\right\|_{1, m}^{-1}}{n(n+m-1)}\left(1-P_{n}^{m}(h)\right), & n=1,2, \ldots \\ 1, & n=0\end{cases}
$$

Demonstração: Segue diretamente do lema anterior que $G_{h} \in L^{1, m}\left([-1,1], d w_{m}\right), h \in$ $(-1,1)$. Para a outra parte, como $G_{h}(t) \geq 0, t \in(-1,1)$, já temos que

$$
G_{h} \widehat{\Upsilon}(0)=\left\|G_{h}\right\|_{1, m}=1 \text {. }
$$

Se $n \geq 1$,

$$
G_{h} \widehat{ }(n)=\left\|\mathcal{L}_{h}\right\|_{1, m}^{-1} \mathcal{L}_{h} \widehat{r}(n)=\frac{1-P_{n}^{m}(h)}{n(n+m-1)}\left\|\mathcal{L}_{h}\right\|_{1, m}^{-1}
$$

pelo lema anterior novamente.

Os próximos três lemas contêm algumas propriedades adicionais da função $\mathcal{L}_{h}$.

Lema 2.5.4. Se $h \in[0,1)$, então $\left\|\mathcal{L}_{h}\right\|_{1, m} \leq 1-h$.

Demonstração: Basta mostrar que a função

$$
\psi(h)=\left\|\mathcal{L}_{h}\right\|_{1, m}-(1-h), \quad h \in[0,1),
$$

é não-positiva. Fazendo uma mudança de variáveis, obtemos

$$
\begin{aligned}
\left\|\mathcal{L}_{h}\right\|_{1, m} & =\int_{h}^{1}\left(\int_{h}^{t}\left(1-u^{2}\right)^{-m / 2} d u\right) d w_{m}(t) \\
& =\int_{h}^{1} \int_{u}^{1}\left(1-u^{2}\right)^{-m / 2} d w_{m}(t) d u .
\end{aligned}
$$

Como $\lim _{h \rightarrow 1^{-}} \psi(h)=0$, é suficiente mostrar que $\psi(h)$ é não-decrescente no intervalo $[0,1)$. Como

$$
\psi^{\prime}(h)=1-\left(1-h^{2}\right)^{-m / 2} \int_{h}^{1} d w_{m}(t)
$$

basta mostrarmos que

$$
\int_{h}^{1} d w_{m}(t) \leq\left(1-h^{2}\right)^{m / 2}
$$

Mas, pelo Teorema do Valor Médio para integrais, sabemos que

$$
\int_{h}^{1} d w_{m}(t)=\int_{h}^{1}\left(1-t^{2}\right)^{(m-2) / 2} d t=\left(1-\eta^{2}\right)^{(m-2) / 2}(1-h),
$$

para algum $\eta \in[h, 1)$. Daí,

$$
\int_{h}^{1} d w_{m}(t) \leq\left(1-h^{2}\right)^{(m-2) / 2}(1-h)=\frac{1}{1+h}\left(1-h^{2}\right)^{m / 2} \leq\left(1-h^{2}\right)^{m / 2} .
$$

Isso completa a prova do lema. 
Lema 2.5.5. Se $h \in(-1,1)$, então $m\left\|\mathcal{L}_{h}\right\|_{1, m} \geq 1-h$.

Demonstração: Sejam $\theta, \xi:(-1,1) \rightarrow \mathbb{R}$ as funções definidas por $\theta(h):=1-h$ e

$$
\xi(h):=m\left\|\mathcal{L}_{h}\right\|_{1, m}=m \int_{h}^{1}\left(1-u^{2}\right)^{-m / 2}\left(\int_{u}^{1} d w_{m}(t)\right) d u .
$$

Usando o lema anterior, é fácil ver que $\lim _{h \rightarrow 1^{-}} \xi(h)-\theta(h)=0$. Logo, para provar o lema, é suficiente mostrar que $\xi^{\prime}(h) \leq \theta^{\prime}(h), h \in(-1,1)$, ou seja, que

$$
-m\left(1-h^{2}\right)^{-m / 2} \int_{h}^{1} d w_{m}(t) \leq-1, \quad h \in(-1,1) .
$$

Definindo-se

$$
\psi(h):=m \int_{h}^{1} d w_{m}(t)-\left(1-h^{2}\right)^{m / 2}, \quad h \in(-1,1),
$$

é, então, suficiente mostrar que $\psi(h) \geq 0, h \in(-1,1)$. Como $\lim _{h \rightarrow 1^{-}} \psi(h)=0 \mathrm{e}$

$$
\begin{aligned}
\psi^{\prime}(h) & =-m\left(1-h^{2}\right)^{(m-2) / 2}+m h\left(1-h^{2}\right)^{(m-2) / 2} \\
& =m\left(1-h^{2}\right)^{(m-2) / 2}(-1+h) \leq 0, \quad h \in(-1,1) .
\end{aligned}
$$

o resultado segue.

Lema 2.5.6. Se $\mathcal{L}_{h}$ é a função definida no Lema 2.5.2, então

$$
\lim _{h \rightarrow 1} \frac{m\left\|\mathcal{L}_{h}\right\|_{1, m}}{1-h}=1
$$

Demonstração: Notemos primeiramente que fazendo uma mudança de variáveis obtemos

$$
\begin{aligned}
\left\|\mathcal{L}_{h}\right\|_{1, m} & =\frac{\sigma_{m-1}}{\sigma_{m}} \int_{-1}^{1} \mathcal{L}_{h}(t) d w_{m}(t) \\
& =\int_{h}^{1} \int_{h}^{t}\left(1-u^{2}\right)^{-m / 2} d u d w_{m}(t) \\
& =\int_{h}^{1} \int_{u}^{1}\left(1-u^{2}\right)^{-m / 2} d w_{m}(t) d u .
\end{aligned}
$$

Logo, usando a Regra de L'Hospital duas vezes, segue que

$$
\begin{aligned}
\lim _{h \rightarrow 1} \frac{m\left\|\mathcal{L}_{h}\right\|_{1, m}}{1-h} & =\lim _{h \rightarrow 1} \frac{m}{1-h} \int_{h}^{1} \int_{u}^{1}\left(1-u^{2}\right)^{-m / 2} d w_{m}(t) d u \\
& =\lim _{h \rightarrow 1} \frac{m}{\left(1-h^{2}\right)^{m / 2}} \int_{h}^{1} d w_{m}(t) \\
& =\lim _{h \rightarrow 1} \frac{1}{h} \\
& =1
\end{aligned}
$$

Isso conclui a prova. 


\subsection{O operador $A_{h}^{r}$}

No que segue, usaremos a função $G_{h}$ para construir um operador de convolução esférica associado, aqui chamado de operador auxiliar. O operador está relacionado diretamente com outros até agora introduzidos, e será usado na dedução de várias propriedades subseqüentes, dentre elas caracterizações para os espaços $W_{X}^{r}$ e propriedades do módulo de suavidade que introduziremos à frente.

Definição 2.6.1. Sejam $r$ um inteiro positivo e $h \in(-1,1)$. O operador auxiliar é o operador linear $A_{h}^{r}: X \rightarrow X$ dado por

$$
A_{h}^{1}(f):=G_{h} * f, \quad f \in X
$$

e, indutivamente, por

$$
A_{h}^{r}:=A_{h}^{1} \circ A_{h}^{r-1}, \quad r=2,3, \ldots
$$

As propriedades básicas do operador $A_{h}^{r}$ estão listadas no lema abaixo.

Lema 2.6.2. Sejam $h \in(-1,1)$ e $r$ um inteiro positivo. Então $A_{h}^{r}$ é um operador positivo com norma $\left\|A_{h}^{r}\right\|_{X}=1$. Além disso,

$$
\left\|f-A_{h}^{r}(f)\right\|_{X} \leq r \sup _{h \leq t<1}\left\|f-S_{t}^{m}(f)\right\|_{X}, \quad f \in X
$$

Em particular,

$$
\lim _{h \rightarrow 1^{-}}\left\|f-A_{h}^{r}(f)\right\|_{X}=0, \quad f \in X .
$$

Demonstração: Como $G_{h}$ é uma função não-negativa, a positividade de $A_{h}^{r}$ segue. Usando a Proposição 1.2.5 e o Lema 2.5.3, temos que

$$
\begin{aligned}
\left\|A_{h}^{r}(f)\right\|_{X} & =\left\|G_{h} *\left(A_{h}^{r-1}(f)\right)\right\|_{X} \\
& \leq\left\|G_{h}\right\|_{1, m}\left\|A_{h}^{r-1}(f)\right\|_{X} \\
& \vdots \\
& \leq\left\|G_{h}\right\|_{1, m}^{r}\|f\|_{X} \\
& =\|f\|_{X}, \quad f \in X .
\end{aligned}
$$

Mas, se $f_{0}=1$, então como $S_{h}^{m}\left(f_{0}\right)=1$, usando o Teorema 2.2.10, obtemos

$$
A_{h}^{1}\left(f_{0}\right)(x)=\frac{\sigma_{m-1}}{\sigma_{m}} \int_{-1}^{1} G_{h}(t) d w_{m}(t)=\left\|G_{h}\right\|_{1, m}=1, \quad x \in S^{m} .
$$

Portanto,

$$
A_{h}^{r}\left(f_{0}\right)=A_{h}^{r-1}\left(A_{h}^{1}\left(f_{0}\right)\right)=A_{h}^{r-1}\left(f_{0}\right)=\cdots=f_{0}=1 .
$$


Os argumentos acima mostram que $\left\|A_{h}^{r}\left(f_{0}\right)\right\|_{X}=1$. Logo,

$$
\left\|A_{h}^{r}\right\|_{X}=\sup _{f \in X \backslash\{0\}} \frac{\left\|A_{h}^{r}(f)\right\|_{X}}{\|f\|_{X}}=1 .
$$

A definição de $A_{h}^{1}$ e o Teorema 2.2.10 implicam que

$$
\begin{aligned}
\left\|f-A_{h}^{1}(f)\right\|_{X} & =\left\|f-G_{h} * f\right\|_{X} \\
& =\frac{\sigma_{m-1}}{\sigma_{m}}\left\|\int_{-1}^{1}\left[f(\cdot)-S_{t}^{m}(f)(\cdot)\right] G_{h}(t) d w_{m}(t)\right\|_{X} .
\end{aligned}
$$

Logo, usando a desigualdade de Minkowski para integrais, temos que

$$
\begin{aligned}
\left\|f-A_{h}^{1}(f)\right\|_{X} & \leq \frac{\sigma_{m-1}}{\sigma_{m}} \int_{-1}^{1}\left\|f-S_{t}^{m}(f)\right\|_{X} G_{h}(t) d w_{m}(t) \\
& =\frac{\sigma_{m-1}}{\sigma_{m}} \int_{h}^{1}\left\|f-S_{t}^{m}(f)\right\|_{X} G_{h}(t) d w_{m}(t) .
\end{aligned}
$$

Agora está claro que

$$
\begin{aligned}
\left\|f-A_{h}^{1}(f)\right\|_{X} & \leq \sup _{h \leq t<1}\left\|f-S_{t}^{m}(f)\right\|_{X} \frac{\sigma_{m-1}}{\sigma_{m}} \int_{h}^{1} G_{h}(t) d w_{m}(t) \\
& =\sup _{h \leq t<1}\left\|f-S_{t}^{m}(f)\right\|_{X}\left\|G_{h}\right\|_{1, m} \\
& =\sup _{h \leq t<1}\left\|f-S_{t}^{m}(f)\right\|_{X} .
\end{aligned}
$$

Se $r \geq 1$, usando o fato de que $\left\|A_{h}^{r}\right\|_{X}=1$ e a parte anterior, vemos que

$$
\begin{aligned}
\left\|f-A_{h}^{r}(f)\right\|_{X} & =\left\|f-A_{h}^{1}(f)+\sum_{j=1}^{r-1}\left(A_{h}^{j}(f)-A_{h}^{j+1}(f)\right)\right\|_{X} \\
& \leq\left\|f-A_{h}^{1}(f)\right\|_{X}+\sum_{j=1}^{r-1}\left\|A_{h}^{j}(f)-A_{h}^{j+1}(f)\right\|_{X} \\
& =\left\|f-A_{h}^{1}(f)\right\|_{X}+\sum_{j=1}^{r-1}\left\|A_{h}^{j}\left(f-A_{h}^{1}(f)\right)\right\|_{X} \\
& \leq\left\|f-A_{h}^{1}(f)\right\|_{X}+\sum_{j=1}^{r-1}\left\|f-A_{h}^{1}(f)\right\|_{X} \\
& =r\left\|f-A_{h}^{1}(f)\right\|_{X} \\
& =r \sup _{h \leq t<1}\left\|f-S_{t}^{m}(f)\right\|_{X} .
\end{aligned}
$$

Ainda, como $\lim _{t \rightarrow 1^{-}}\left\|f-S_{t}^{m}(f)\right\|_{X}=0$ (Proposição 2.2.6- $(i)$ ), deduzimos que

$$
\lim _{h \rightarrow 1^{-}}\left\|f-A_{h}^{r}(f)\right\|_{X} \leq \lim _{h \rightarrow 1^{-}} r \sup _{h \leq t<1}\left\|f-S_{t}^{m}(f)\right\|_{X}=0,
$$

e o lema está provado.

No lema abaixo analisamos o efeito do operador projeção sobre o operador $A_{h}^{r}$. 
Lema 2.6.3. Sejam $r$ um inteiro positivo e $h \in(-1,1)$. Então vale a igualdade

$$
\mathcal{Y}_{n} \circ A_{h}^{r}=\left(G_{h} \widehat{(}(n)\right)^{r} \mathcal{Y}_{n}, \quad n \in \mathbb{N} \text {. }
$$

Em particular,

$$
\mathcal{Y}_{n} \circ\left(I-A_{h}^{r}\right)^{r-1}=\left[1-\left(G_{h} \widehat{(}(n)\right)^{r}\right]^{r-1} \mathcal{Y}_{n}, \quad n \in \mathbb{N}
$$

Demonstração: Seja $f \in X$. Então a Proposição 2.1.4 implica que

$$
\begin{aligned}
& \mathcal{Y}_{n}\left(A_{h}^{r}(f)\right)=\mathcal{Y}_{n}\left(A_{h}^{1}\left(A_{h}^{r-1}(f)\right)\right) \\
& =G_{h} \widehat{(}(n) \mathcal{Y}_{n}\left(A_{h}^{r-1}(f)\right) \\
& =\left(G_{h} \widehat{(}(n)\right)^{r} \mathcal{Y}_{n}(f), \quad n \in \mathbb{N} .
\end{aligned}
$$

Usando a linearidade de $\mathcal{Y}_{n}$ temos que

$$
\begin{aligned}
\mathcal{Y}_{n}\left(\left(I-A_{h}^{r}\right)^{r-1}(f)\right) & =\mathcal{Y}_{n}\left(\left(I-A_{h}^{r}\right) \circ\left(I-A_{h}^{r}\right)^{r-2}(f)\right) \\
& =\mathcal{Y}_{n}\left(\left(I-A_{h}^{r}\right)^{r-2}(f)\right)-\mathcal{Y}_{n}\left(A_{h}^{r}\left(\left(I-A_{h}^{r}\right)^{r-2}(f)\right)\right) \\
& =\mathcal{Y}_{n}\left(\left(I-A_{h}^{r}\right)^{r-2}(f)\right)-\left(G_{h} \widehat{(}(n)\right)^{r} \mathcal{Y}_{n}\left(\left(I-A_{h}^{r}\right)^{r-2}(f)\right) \\
& =\left[1-\left(G_{h} \widehat{(}(n)\right)^{r}\right] \mathcal{Y}_{n}\left(\left(I-A_{h}^{r}\right)^{r-2}(f)\right) \\
& \vdots \\
& =\left[1-\left(G_{h} \widehat{ }(n)\right)^{r}\right]^{r-1} \mathcal{Y}_{n}(f), \quad n \in \mathbb{N} .
\end{aligned}
$$

Isso completa a prova do lema.

Uma propriedade análoga àquela do lema anterior, mas com o operador diferença esférica no lugar de $\mathcal{Y}_{n}$, é como segue.

Lema 2.6.4. Se r é um inteiro positivo, então

$$
\Delta_{t} \circ\left(I-A_{h}^{r}\right)^{r-1}=\left(I-A_{h}^{r}\right)^{r-1} \circ \Delta_{t}, \quad t, h \in(-1,1) .
$$

Demonstração: Seja $f \in X$. Usando o lema anterior e a Proposição 2.2.8-(iii), temos que

$$
\begin{aligned}
& \mathcal{Y}_{n}\left(\Delta_{t}\left(\left(I-A_{h}^{r}\right)^{r-1}(f)\right)=\left(1-P_{n}^{m}(t)\right) \mathcal{Y}_{n}\left(\left(I-A_{h}^{r}\right)^{r-1} f\right)\right. \\
& =\left[1-\left(G_{h} \widehat{(}(n)\right)^{r}\right]^{r-1}\left(1-P_{n}^{m}(t)\right) \mathcal{Y}_{n}(f) \\
& \left.=\left[1-\left(G_{h} \widehat{(n}\right)\right)^{r}\right]^{r-1} \mathcal{Y}_{n}\left(\Delta_{t}(f)\right) \\
& =\mathcal{Y}_{n}\left(\left(I-A_{h}^{r}\right)^{r-1}\left(\Delta_{t}(f)\right)\right), \quad n \in \mathbb{N} \text {. }
\end{aligned}
$$

Logo, o Teorema 2.1.2 implica que

$$
\Delta_{t}\left(\left(I-A_{h}^{r}\right)^{r-1}(f)\right)=\left(I-A_{h}^{r}\right)^{r-1}\left(\Delta_{t}(f)\right),
$$

e a igualdade do lema segue. 
Teorema 2.6.5. Sejam $r$ um inteiro positivo e $h \in(-1,1)$. Valem as seguintes igualdades:

(i) $A_{h}^{r}=m^{-r}\left\|\mathcal{L}_{h}\right\|_{1, m}^{-r}\left(\Delta_{h}^{r} \circ J^{r}\right)+\mathcal{Y}_{0}$

(ii) $A_{h}^{r}=m^{-r}\left\|\mathcal{L}_{h}\right\|_{1, m}^{-r}\left(J^{r} \circ \Delta_{h}^{r}\right)+\mathcal{Y}_{0}$

(iii) $\Delta_{h}^{r} \circ J^{r}=J^{r} \circ \Delta_{h}^{r}$.

Demonstração: Seja $f \in X$. Usando a Proposição 2.1.4, o Lema 2.5.3 e o Teorema 2.4.2, obtemos

$$
\begin{aligned}
& \mathcal{Y}_{n}\left(A_{h}^{r}(f)\right)=\left(G_{h} \widehat{(}(n)\right)^{r} \mathcal{Y}_{n}(f) \\
& =\left(\frac{\left\|\mathcal{L}_{h}\right\|_{1, m}^{-1}}{n(n+m-1)}\left(1-P_{n}^{m}(h)\right)\right)^{r} \mathcal{Y}_{n}(f) \\
& =m^{-r}\left\|\mathcal{L}_{h}\right\|_{1, m}^{-r}\left(\frac{m}{n(n+m-1)}\right)^{r}\left(1-P_{n}^{m}(h)\right)^{r} \mathcal{Y}_{n}(f)
\end{aligned}
$$

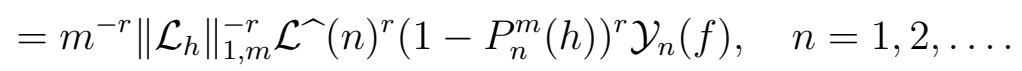

Logo, o Teorema 2.4.4, a Proposição 2.2.8-(iii) e a linearidade de $\mathcal{Y}_{n}$ implicam que

$$
\begin{aligned}
\mathcal{Y}_{n}\left(A_{h}^{r}(f)\right) & =m^{-r}\left\|\mathcal{L}_{h}\right\|_{1, m}^{-r}\left(1-P_{n}^{m}(h)\right)^{r} \mathcal{Y}_{n}\left(J^{r}(f)\right) \\
& =m^{-r}\left\|\mathcal{L}_{h}\right\|_{1, m}^{-r} \mathcal{Y}_{n}\left(\Delta_{h}^{r}\left(J^{r}(f)\right)\right) \\
& =\mathcal{Y}_{n}\left(m^{-r}\left\|\mathcal{L}_{h}\right\|_{1, m}^{-r} \Delta_{h}^{r}\left(J^{r}(f)\right)\right), \quad n=1,2, \ldots
\end{aligned}
$$

Em particular, $A_{h}^{r}(f)-m^{-r}\left\|\mathcal{L}_{h}\right\|_{1, m}^{-r} \Delta_{h}^{r}\left(J^{r}(f)\right)$ é uma constante. Usando a linearidade de $\mathcal{Y}_{0}$, as Proposições 2.1.4 e 2.2.8 obtemos

$$
\mathcal{Y}_{0}\left(A_{h}^{r}(f)-m^{-r}\left\|\mathcal{L}_{h}\right\|_{1, m}^{-r} \Delta_{h}^{r}\left(J^{r}(f)\right)\right)=\mathcal{Y}_{0}(f)
$$

Logo, a constante é $\mathcal{Y}_{0}(f)$. Isso prova a parte $(i)$. A prova de (ii) segue o mesmo padrão e será omitida. A última afirmação do teorema é uma conseqüência de $(i)$ e $(i i)$.

\subsection{Uma caracterização para $W_{X}^{r}$}

Aqui, caracterizaremos os espaços $W_{X}^{r}$ usando os operadores $J^{r}$ e $D^{r}$ e a conexão com o operador $A_{h}^{r}$ dada pelo Teorema 2.6.5. Começamos com uma condição suficiente sobre $J^{r}$ para garantir que uma função $f \in X$ pertença a $W_{X}^{r}$.

Teorema 2.7.1. Seja r um inteiro positivo. Então a imagem do operador $J^{r}$ é um subconjunto de $W_{X}^{r}$. 
Demonstração: Consideremos primeiro o caso $r=1$. Sejam $f, g_{2} \in X$ tais que $f=J^{r}\left(g_{2}\right)$. Usando o Teorema 2.6.5, temos que

$$
\begin{aligned}
\frac{f-S_{h}^{m}(f)}{1-h} & =\frac{J^{1}\left(g_{2}\right)-S_{h}^{m}\left(J^{1}\left(g_{2}\right)\right)}{1-h} \\
& =\left(m\left\|\mathcal{L}_{h}\right\|_{1, m}\right)^{-1}\left(J^{1}\left(g_{2}\right)-S_{h}^{m}\left(J^{1}\left(g_{2}\right)\right)\right) \frac{m\left\|\mathcal{L}_{h}\right\|_{1, m}}{1-h} \\
& =\left(m\left\|\mathcal{L}_{h}\right\|_{1, m}\right)^{-1} \Delta_{h}\left(J^{1}\left(g_{2}\right)\right) \frac{m\left\|\mathcal{L}_{h}\right\|_{1, m}}{1-h} \\
& =\left(A_{h}^{1}\left(g_{2}\right)-\mathcal{Y}_{0}\left(g_{2}\right)\right) \frac{m\left\|\mathcal{L}_{h}\right\|_{1, m}}{1-h} .
\end{aligned}
$$

Conseqüentemente,

$$
\begin{aligned}
\left\|\frac{\Delta_{h}(f)}{1-h}-\left(g_{2}-\mathcal{Y}_{0}\left(g_{2}\right)\right)\right\|_{X} & =\left\|\left(A_{h}^{1}\left(g_{2}\right)-\mathcal{Y}_{0}\left(g_{2}\right)\right) \frac{m\left\|\mathcal{L}_{h}\right\|_{1, m}}{1-h}-\left(g_{2}-\mathcal{Y}_{0}\left(g_{2}\right)\right)\right\|_{X} \\
& \leq\left|\frac{m\left\|\mathcal{L}_{h}\right\|_{1, m}}{1-h}-1\right|\left\|g_{2}-\mathcal{Y}_{0}\left(g_{2}\right)\right\|_{X}+\frac{m\left\|\mathcal{L}_{h}\right\|_{1, m}}{1-h}\left\|A_{h}^{1}\left(g_{2}\right)-g_{2}\right\|_{X} .
\end{aligned}
$$

Aplicando o limite na desigualdade acima para $h \rightarrow 1^{-}$e usando ambos, o Lema 2.6.2 e o Lema 2.5.6, obtemos

$$
\lim _{h \rightarrow 1^{-}}\left\|\frac{\Delta_{h}(f)}{1-h}-\left(g_{2}-\mathcal{Y}_{0}\left(g_{2}\right)\right)\right\|_{X}=0 .
$$

Logo, $f \in W_{X}^{1}$ e $D^{1}(f)=g_{2}-\mathcal{Y}_{0}\left(g_{2}\right)$. Para completar a prova, vamos assumir, indutivamente, que o teorema vale nos casos $r=1,2, \ldots, s-1$. Sejam $f, g_{2} \in X$ tal que $J^{s}\left(g_{2}\right)=f$. Como $J^{1}\left(J^{s-1}\left(g_{2}\right)\right)=f$, a hipótese de indução implica que $f \in W_{X}^{s-1}$ e

$$
D^{1}(f)=J^{s-1}\left(g_{2}\right)-\mathcal{Y}_{0}\left(J^{s-1}\left(g_{2}\right)\right)
$$

Está claro agora que $J^{s-1}\left(g_{2}\right) \in X$. A hipótese de indução, novamente, implica que

$$
D^{1}(f)+\mathcal{Y}_{0}\left(J^{s-1}\left(g_{2}\right)\right) \in W_{X}^{s-1} .
$$

Como $W_{X}^{s-1}$ é um espaço vetorial, isso implica que $D^{1}(f) \in W_{X}^{s-1}$. Assim, $f \in W_{X}^{s}$.

O resultado principal dessa seção é como segue.

Teorema 2.7.2. Sejam $r$ um inteiro positivo e $f \in X$. As seguintes afirmações são equivalentes:

(i) $f \in W_{X}^{r}$;

(ii) Existe uma função $g_{1} \in X$ tal que

$$
\mathcal{Y}_{n}\left(g_{1}\right)=\left(\frac{n(n+m-1)}{m}\right)^{r} \mathcal{Y}_{n}(f), \quad n=0,1, \ldots ;
$$

(iii) Existe uma única função $g_{2} \in X$ tal que

$$
J^{r}\left(g_{2}\right)=f
$$


Demonstração: Para justificar que $(i)$ implica $(i i)$ é suficiente tomar $g_{1}=D^{r}(f)$ no Teorema 2.3.6. Se $(i i)$ vale para alguma função $g_{1} \in X$, o Teorema 2.4 .4 implica que

$$
\mathcal{Y}_{n}(f)=\left(\frac{m}{n(n+m-1)}\right)^{r} \mathcal{Y}_{n}\left(g_{1}\right)=\mathcal{Y}_{n}\left(J^{r}\left(g_{1}\right)\right), \quad n=1,2, \ldots
$$

Definindo $g_{2}=g_{1}+\mathcal{Y}_{0}(f)$ podemos usar a Proposição 2.1.1 e o Teorema 2.4.4, novamente, para deduzir que

$$
\mathcal{Y}_{n}\left(J^{r}\left(g_{2}\right)\right)=\mathcal{Y}_{n}\left(J^{r}\left(g_{1}\right)\right)+\mathcal{Y}_{n}\left(J^{r}\left(\mathcal{Y}_{0}(f)\right)=\mathcal{Y}_{n}(f)\right.
$$

Um cálculo semelhante e o fato de que $\mathcal{Y}_{0}\left(g_{1}\right)=0$, mostram que a igualdade anterior também é válida para $n=0$. Logo, $J^{r}\left(g_{2}\right)=f$. Finalmente, se $J^{r}\left(g_{2}\right)=J^{r}\left(f_{2}\right)$ para duas funções $g_{2}$ e $f_{2}$, então

$$
\mathcal{Y}_{n}\left(J^{r}\left(g_{2}\right)\right)=\mathcal{Y}_{n}\left(J^{r}\left(f_{2}\right)\right), \quad n=0,1, \ldots
$$

Do Teorema 2.4.4, segue que

$$
\mathcal{Y}_{n}\left(g_{2}\right)=\mathcal{Y}_{n}\left(f_{2}\right), \quad n=0,1, \ldots
$$

Logo, o Teorema 2.1.2 implica que $g_{2}=f_{2}$. Finalmente, o teorema anterior completa a prova do teorema.

O próximo resultado fornece uma descrição do espaço $W_{X}^{r}$ em termos do operador integral de Laplace-Beltrami.

Corolário 2.7.3. Se r é um inteiro positivo, então $W_{X}^{r}=X \cap J^{r}(X)$.

O Corolário acima induz uma norma alternativa no espaço $W_{X}^{r}$. Precisamente, a expressão

$$
|f|_{W_{X}^{r}}=\|f\|_{X}+\|f\|_{J^{r}(X)}, \quad f \in W_{X}^{r},
$$

onde $\|f\|_{J^{r}(X)}:=\|g\|_{X}$ e $g \in X$ é tal que $f=J^{r}(g)$, define uma norma em $W_{X}^{r}$.

Fecharemos a seção apresentando aplicações do Teorema 2.7.2 que envolvem a derivada forte de Laplace-Beltrami.

Proposição 2.7.4. Valem as seguintes afirmações:

(i) Se $r_{1}, r_{2}$ são inteiros positivos e $r_{2}<r_{1}$, então $W_{X}^{r_{1}} \subset W_{X}^{r_{2}}$.

(ii) Se r é um inteiro positivo, $f \in W_{X}^{r}$ e $K \in L^{1, m}\left([-1,1], d w_{m}\right)$, então $K * f \in W_{X}^{r}$ e

$$
D^{r}(K * f)=K * D^{r}(f) .
$$

Demonstração: Sejam $r_{1}$ e $r_{2}$ como em $(i)$ e $f \in W_{X}^{r_{1}}$. Se $g_{1}$ é a função definida por

$$
g_{1}=J^{r_{1}-r_{2}}\left(D^{r_{1}}(f)\right)
$$


aplicando os Teoremas 2.3.6 e 2.4.4, vem que

$$
\begin{aligned}
\mathcal{Y}_{n}\left(J^{r_{1}-r_{2}}\left(D^{r_{1}}(f)\right)\right. & =\left(\frac{m}{n(n+m-1)}\right)^{r_{1}-r_{2}} \mathcal{Y}_{n}\left(D^{r_{1}}(f)\right) \\
& =\left(\frac{n(n+m-1)}{m}\right)^{r_{2}-r_{1}}\left(\frac{n(n+m-1)}{m}\right)^{r_{1}} \mathcal{Y}_{n}(f) \\
& =\left(\frac{n(n+m-1)}{m}\right)^{r_{2}} \mathcal{Y}_{n}(f), \quad n=1,2, \ldots
\end{aligned}
$$

Como $\mathcal{Y}_{0} \circ J^{r_{1}-r_{2}}=\mathcal{Y}_{0}$ (Teorema 2.4.4), a igualdade acima também é válida para $n=0$. Logo, o teorema anterior implica que $f \in W_{X}^{r_{2}}$. Para a outra parte sejam $r$ um inteiro positivo, $f \in W_{X}^{r}$ e $K \in L^{1, m}\left([-1,1], d w_{m}\right)$. A Proposição 2.1.4 implica que

$$
\begin{aligned}
\mathcal{Y}_{n}\left(K * D^{r}(f)\right) & =K^{\Upsilon}(n) \mathcal{Y}_{n}\left(D^{r}(f)\right) \\
& =K^{\curlywedge}(n)\left(\frac{n(n+m-1)}{m}\right)^{r} \mathcal{Y}_{n}(f) \\
& =\left(\frac{n(n+m-1)}{m}\right)^{r} \mathcal{Y}_{n}(K * f), \quad n \in \mathbb{N} .
\end{aligned}
$$

Como $K * D^{r}(f) \in X$, o Teorema 2.7.2 garante que $K * f \in W_{X}^{r}$. Usando o Teorema 2.3.6 temos que

$$
\mathcal{Y}_{n}\left(K * D^{r}(f)\right)=\left(\frac{n(n+m-1)}{m}\right)^{r} \mathcal{Y}_{n}(K * f)=\mathcal{Y}_{n}\left(D^{r}(K * f)\right), \quad n \in \mathbb{N}
$$

Portanto, o Teorema 2.1.2 conclui a prova da proposição.

Teorema 2.7.5. Se r é um inteiro positivo, então $D^{r} \circ J^{r}=I-\mathcal{Y}_{0}$.

Demonstração: Sejam $r$ um inteiro positivo e $f \in X$. Se $g_{1}=f-\mathcal{Y}_{0}(f) \in X$, então do Teorema 2.4.4, segue que

$$
\begin{aligned}
\mathcal{Y}_{n}\left(g_{1}\right) & =\mathcal{Y}_{n}(f)-\mathcal{Y}_{n}\left(\mathcal{Y}_{0}(f)\right) \\
& =\mathcal{Y}_{n}(f) \\
& =\left(\frac{n(n+m-1)}{m}\right)^{r}\left(\frac{m}{n(n+m-1)}\right)^{r} \mathcal{Y}_{n}(f) \\
& =\left(\frac{n(n+m-1)}{m}\right)^{r} \mathcal{Y}_{n}\left(J^{r}(f)\right), \quad n=1,2, \ldots
\end{aligned}
$$

Como $\mathcal{Y}_{0}$ é projeção, a mesma igualdade vale no caso $n=0$. Logo, o Teorema 2.7.2 garante que $J^{r}(f) \in W_{X}^{r}$. Usando o Teorema 2.3.6, obtemos

$$
\mathcal{Y}_{n}\left(f-\mathcal{Y}_{0}(f)\right)=\mathcal{Y}_{n}\left(D^{r}\left(J^{r}(f)\right)\right), \quad n \in \mathbb{N},
$$

e, conseqüentemente, $D^{r}\left(J^{r}(f)\right)=f-\mathcal{Y}_{0}(f)$.

Combinando os Teoremas 2.4.5 e 2.7.5, obtemos o seguinte resultado. 
Corolário 2.7.6. Se r é um inteiro positivo, então $D^{r} \circ J^{r}=J^{r} \circ D^{r}$ em $W_{X}^{r}$.

O Teorema 2.7.2 mostra que o operador $J^{r}$ é o operador inverso da derivada forte de Laplace-Beltrami $D^{r}$, a menos de uma constante. Juntando-se o corolário anterior e o Corolário 2.4.6, obtemos o resultado a seguir.

Corolário 2.7.7. Se r é um inteiro positivo, então as igualdades $D^{r} \circ J^{r}=J^{r} \circ D^{r}=I$ valem em $\left[\cup_{k=1}^{\infty} \mathcal{H}_{k}\left(S^{m}\right)\right]$.

A equivalência das duas normas introduzidas no espaço $W_{X}^{r}$ é justificada na próxima proposição.

Proposição 2.7.8. Seja $r$ um inteiro positivo. Então as normas $\|\cdot\|_{W_{X}^{r}} e|\cdot|_{W_{X}^{r}}$ são equivalentes.

Demonstração: Seja $f \in W_{X}^{r}$ e $g \in X$ tal que $J^{r}(g)=f$. Usando o Teorema 2.7.5, temos que

$$
\left\|D^{r}(f)\right\|_{X}=\left\|D^{r}\left(J^{r}(g)\right)\right\|_{X}=\left\|g-\mathcal{Y}_{0}(g)\right\|_{X} \leq\|g\|_{X}+\left\|\mathcal{Y}_{0}(g)\right\|_{X} .
$$

Pelo Teorema 2.4.4 e pela Proposição 2.1.3-(iii), obtemos

$$
\left\|D^{r}(f)\right\|_{X} \leq\|g\|_{X}+\left\|\mathcal{Y}_{0}\left(J^{r}(g)\right)\right\|_{X}=\|g\|_{X}+\left\|\mathcal{Y}_{0}(f)\right\|_{X} \leq\|f\|_{J^{r}(X)}+\|f\|_{X} .
$$

Logo,

$$
\|f\|_{W_{X}^{r}}=\|f\|_{X}+\left\|D^{r}(f)\right\|_{X} \leq 2\|f\|_{X}+\|f\|_{J^{r}(X)} \leq 2\left(\|f\|_{X}+\|f\|_{J^{r}(X)}\right)=2|f|_{W_{X}^{r}} .
$$

Por outro lado, usando novamente o Teorema 2.7.5, segue que

$$
|f|_{W_{X}^{r}}=\|f\|_{X}+\|g\|_{X}=\|f\|_{X}+\left\|D^{r}\left(J^{r}(g)\right)+\mathcal{Y}_{0}(g)\right\|_{X} \leq\|f\|_{X}+\left\|D^{r}(f)\right\|_{X}+\left\|\mathcal{Y}_{0}(g)\right\|_{X} .
$$

Portanto, pelo Teorema 2.4.4 e pela Proposição 2.1.3-(iii), vem que

$$
\begin{aligned}
|f|_{W_{X}^{r}} & \leq\|f\|_{X}+\left\|D^{r}(f)\right\|_{X}+\left\|\mathcal{Y}_{0}\left(J^{r}(g)\right)\right\|_{X} \\
& =\|f\|_{X}+\left\|D^{r}(f)\right\|_{X}+\left\|\mathcal{Y}_{0}(f)\right\|_{X} \\
& \leq 2\|f\|_{X}+\left\|D^{r}(f)\right\|_{X} \\
& \leq 2\|f\|_{W_{X}^{r}} .
\end{aligned}
$$

Isso completa a prova da proposição.

No próximo resultado, usamos o Teorema 2.7.2 para calcular a derivada forte de LaplaceBeltrami do operador $A_{h}^{r}$. Antes, porém, precisamos de uma pequena adaptação do Teorema 2.6.5-(ii). 
Lema 2.7.9. Nas condições do Teorema 2.6.5

$$
A_{h}^{r}=J^{r} \circ\left(\left(m^{-r}\left\|\mathcal{L}_{h}\right\|_{1, m}^{-r}\right) \Delta_{h}^{r}+\mathcal{Y}_{0}\right)
$$

Demonstração: Seja $f \in X$. Procedendo como na prova do Teorema 2.6.5, temos que

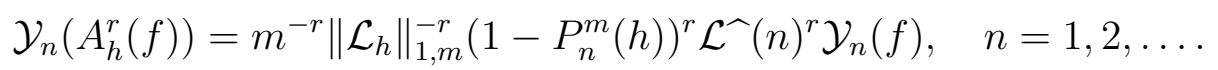

Usando o Teorema 2.4.4 e a Proposição 2.1.1, segue que

$$
\begin{aligned}
& \mathcal{Y}_{n}\left(A_{h}^{r}(f)\right)=m^{-r}\left\|\mathcal{L}_{h}\right\|_{1, m}^{-r} \mathcal{L}^{\Upsilon}(n)^{r} \mathcal{Y}_{n}\left(\Delta_{h}^{r}(f)\right)+\mathcal{Y}_{n}\left(\mathcal{Y}_{0}(f)\right) \\
& =\mathcal{L}^{\prec}(n)^{r} \mathcal{Y}_{n}\left(m^{-r}\left\|\mathcal{L}_{h}\right\|_{1, m}^{-r} \Delta_{h}^{r}(f)\right)+\mathcal{L}^{\prec}(n)^{r} \mathcal{Y}_{n}\left(\mathcal{Y}_{0}(f)\right) \\
& =\mathcal{Y}_{n}\left(J^{r}\left(m^{-r}\left\|\mathcal{L}_{h}\right\|_{1, m}^{-r} \Delta_{h}^{r}(f)\right)\right)+\mathcal{Y}_{n}\left(J^{r}\left(\mathcal{Y}_{0}(f)\right)\right) \\
& =\mathcal{Y}_{n}\left(J^{r}\left(m^{-r}\left\|\mathcal{L}_{h}\right\|_{1, m}^{-r} \Delta_{h}^{r} f+\mathcal{Y}_{0}(f)\right)\right), \quad n=1,2, \ldots
\end{aligned}
$$

Pelos Lemas 2.6.3 e 2.5.3, Proposição 2.1.1 e a definição de $P_{0}^{m}$, temos ainda que

$$
\mathcal{Y}_{0}\left(A_{h}^{r}(f)\right)=\left(G_{h} \widehat{\Upsilon}(0)\right)^{r} \mathcal{Y}_{0}(f)=m^{-r}\left\|\mathcal{L}_{h}\right\|_{1, m}^{-r} \mathcal{L}^{\widehat{\gamma}}(0)^{r}\left(1-P_{0}^{m}(h)\right)^{r} \mathcal{Y}_{0}(f)+\mathcal{Y}_{0}\left(\mathcal{Y}_{0}(f)\right)
$$

Logo, procedendo-se como acima,

$$
\begin{aligned}
& \mathcal{Y}_{0}\left(A_{h}^{r}(f)\right)=m^{-r}\left\|\mathcal{L}_{h}\right\|_{1, m}^{-r} \mathcal{L}^{-}(0)^{r} \mathcal{Y}_{0}\left(\Delta_{h}^{r}(f)\right)+\mathcal{Y}_{0}\left(\mathcal{Y}_{0}(f)\right)
\end{aligned}
$$

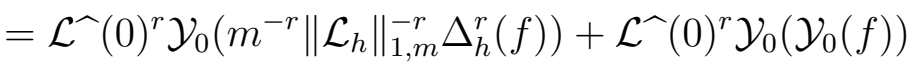

$$
\begin{aligned}
& =\mathcal{Y}_{0}\left(J^{r}\left(m^{-r}\left\|\mathcal{L}_{h}\right\|_{1, m}^{-r} \Delta_{h}^{r}(f)\right)\right)+\mathcal{Y}_{0}\left(J^{r}\left(\mathcal{Y}_{0}(f)\right)\right) \\
& =\mathcal{Y}_{0}\left(J^{r}\left(m^{-r}\left\|\mathcal{L}_{h}\right\|_{1, m}^{-r} \Delta_{h}^{r}(f)+\mathcal{Y}_{0}(f)\right)\right) \text {. }
\end{aligned}
$$

Portanto, o Teorema 2.1.2 completa a prova.

Teorema 2.7.10. Sejam $r$ um inteiro positivo e $h \in(-1,1)$. Valem as seguintes afirmações:

(i) A imagem do operador $A_{h}^{r}$ é um subconjunto de $W_{X}^{r}$;

(ii) $D^{r} \circ A_{h}^{r}=m^{-r}\left\|\mathcal{L}_{h}\right\|_{1, m}^{-r} \Delta_{h}^{r}$.

Demonstração: Se $f \in X$, então $m^{-r}\left\|\mathcal{L}_{h}\right\|_{1, m}^{-r} \Delta_{h}^{r}(f) \in X$. Usando o Teorema 2.4 .4 e a Proposição 2.1.1, temos que

$$
\mathcal{Y}_{n}\left(m^{-r}\left\|\mathcal{L}_{h}\right\|_{1, m}^{-r} \Delta_{h}^{r}(f)\right)=\left(\frac{n(n+m-1)}{m}\right)^{r} \mathcal{Y}_{n}\left(J^{r}\left(m^{-r}\left\|\mathcal{L}_{h}\right\|_{1, m}^{-r} \Delta_{h}^{r}(f)\right)\right)+\mathcal{Y}_{n}\left(\mathcal{Y}_{0}(f)\right)
$$

Pela linearidade de $\mathcal{Y}_{n}$ e o Teorema 2.6.5, deduzimos que

$$
\mathcal{Y}_{n}\left(m^{-r}\left\|\mathcal{L}_{h}\right\|_{1, m}^{-r} \Delta_{h}^{r}(f)\right)=\left(\frac{n(n+m-1)}{m}\right)^{r} \mathcal{Y}_{n}\left(A_{h}^{r}(f)\right), \quad n=1,2, \ldots
$$


A igualdade acima também é válida para $n=0$ devido à Proposição 2.2.8-(iii). Portanto, o Teorema 2.7.2 garante que $A_{h}^{r}(f) \in W_{X}^{r}$. Para provar (ii) usamos o Lema 2.7.9 e o Teorema 2.7.5 para escrever

$$
\begin{aligned}
D^{r} \circ A_{h}^{r} & =D^{r} \circ J^{r} \circ\left(\left(m^{-r}\left\|\mathcal{L}_{h}\right\|_{1, m}^{-r}\right) \Delta_{h}^{r}+\mathcal{Y}_{0}\right) \\
& =m^{-r}\left\|\mathcal{L}_{h}\right\|_{1, m}^{-r} \Delta_{h}^{r}+\mathcal{Y}_{0}-\mathcal{Y}_{0} \circ\left(m^{-r}\left\|\mathcal{L}_{h}\right\|_{1, m}^{-r} \Delta_{h}^{r}+\mathcal{Y}_{0}\right) \\
& =m^{-r}\left\|\mathcal{L}_{h}\right\|_{1, m}^{-r} \Delta_{h}^{r}-\mathcal{Y}_{0} \circ\left(m^{-r}\left\|\mathcal{L}_{h}\right\|_{1, m}^{-r} \Delta_{h}^{r}\right) .
\end{aligned}
$$

Mas,

$$
\mathcal{Y}_{0} \circ\left(m^{-r}\left\|\mathcal{L}_{h}\right\|_{1, m}^{-r} \Delta_{h}^{r}\right)=m^{-r}\left\|\mathcal{L}_{h}\right\|_{1, m}^{-r} \mathcal{Y}_{0} \circ \Delta_{h}^{r}=m^{-r}\left\|\mathcal{L}_{h}\right\|_{1, m}^{-r}\left(1-P_{0}^{m}(h)\right)^{r} \mathcal{Y}_{0}=0
$$

Portanto, $D^{r} \circ A_{h}^{r}=m^{-r}\left\|\mathcal{L}_{h}\right\|_{1, m}^{-r} \Delta_{h}^{r}$.

O próximo resultado é conseqüência da Proposição 2.7.4-(ii).

Corolário 2.7.11. Sejam $r$ um inteiro positivo e $h \in(-1,1)$. Então $D^{r} \circ A_{h}^{r}$ é a r-ésima convolução de $G_{h}$ com $D^{r}$ em $W_{X}^{r}$, isto é, $\left.D^{r} \circ A_{h}^{r}=G_{h} *\left(G_{h} * \cdots *\left(G_{h} * D^{r}\right)\right)\right)$ em $W_{X}^{r}$.

Teorema 2.7.12. Seja $r$ um inteiro positivo. Então o operador $D^{r}: W_{X}^{r} \rightarrow X$ é fechado.

Demonstração: Sejam $f, g \in X$ e $\left\{f_{n}\right\}_{n \in \mathbb{N}} \subset W_{X}^{r}$ uma seqüência tal que

$$
\lim _{n \rightarrow \infty}\left\|f_{n}-f\right\|_{X}=\lim _{n \rightarrow \infty}\left\|D^{r}\left(f_{n}\right)-g\right\|_{X}=0 .
$$

Usando o Teorema 2.3.6 e a Proposição 2.1.3-(ii) temos que

$$
\begin{aligned}
\left|\left(\frac{k(k+m-1)}{m}\right)^{r} \mathcal{Y}_{k}\left(f_{n}\right)-\mathcal{Y}_{k}(g)\right| & =\left|\mathcal{Y}_{k}\left(D^{r}\left(f_{n}\right)\right)-\mathcal{Y}_{k}(g)\right| \\
& =\left|\mathcal{Y}_{k}\left(D^{r}\left(f_{n}\right)-g\right)\right| \\
& \leq N(m, k)\left\|D^{r}\left(f_{n}-g\right)\right\|_{X}, \quad k \in \mathbb{N} .
\end{aligned}
$$

Como $\lim _{n \rightarrow \infty}\left\|D^{r}\left(f_{n}\right)-g\right\|_{X}=0$, segue que

$$
\lim _{n \rightarrow \infty}\left(\frac{k(k+m-1)}{m}\right)^{r} \mathcal{Y}_{k}\left(f_{n}\right)=\mathcal{Y}_{k}(g), \quad k \in \mathbb{N} .
$$

Por outro lado, como

$$
\left|\mathcal{Y}_{k}\left(f_{n}\right)-\mathcal{Y}_{k}(f)\right|=\left|\mathcal{Y}_{k}\left(f_{n}-f\right)\right| \leq N(m, k)\left\|f_{n}-f\right\|_{X}, \quad k \in \mathbb{N},
$$

e $\lim _{n \rightarrow \infty}\left\|f_{n}-f\right\|_{X}=0$, vemos que $\lim _{n \rightarrow \infty} \mathcal{Y}_{k}\left(f_{n}\right)=\mathcal{Y}_{k}(f), k \in \mathbb{N}$. Segue que

$$
\left(\frac{k(k+m-1)}{m}\right)^{r} \mathcal{Y}_{k}(f)=\mathcal{Y}_{k}(g), \quad k \in \mathbb{N} .
$$

Portanto, pelo Teorema 2.7.2, $f \in W_{X}^{r}$ e $D^{r}(f)=g$. Isto justifica o fechamento de $D^{r}$. 
Teorema 2.7.13. Seja r um inteiro positivo. Então o espaço $\left(W_{X}^{r},\|\cdot\|_{W_{X}^{r}}\right)$ é completo.

Demonstração: Seja $\left\{f_{n}\right\}_{n \in \mathbb{N}}$ uma seqüência de Cauchy em $W_{X}^{r}$. Então dado $\epsilon>0$, existe $N \in \mathbb{N}$ tal que

$$
\left\|f_{n}-f_{m}\right\|_{X}+\left\|D^{r}\left(f_{n}\right)-D^{r}\left(f_{m}\right)\right\|_{X}=\left\|f_{n}-f_{m}\right\|_{W_{X}^{r}} \leq \epsilon, \quad n, m \geq N .
$$

Logo, $\left\{f_{n}\right\}_{n \in \mathbb{N}}$ e $\left\{D^{r}\left(f_{n}\right)\right\}_{n \in \mathbb{N}}$ são seqüências de Cauchy em $X$. Como $X$ é completo, existem funções $f, g \in X$ tal que

$$
\lim _{n \rightarrow \infty}\left\|f_{n}-f\right\|_{X}=\lim _{n \rightarrow \infty}\left\|D^{r}\left(f_{n}\right)-g\right\|_{X}=0 .
$$

Portanto, pelo teorema anterior temos que $f \in W_{X}^{r}$ e $D^{r}(f)=g$. Assim,

$$
\lim _{n \rightarrow \infty}\left\|f_{n}-f\right\|_{W_{X}^{r}}=\lim _{n \rightarrow \infty}\left\|f_{n}-f\right\|_{X}+\left\|D^{r}\left(f_{n}\right)-D^{r}(f)\right\|_{X}=0
$$

completando a prova do teorema.

\subsection{O módulo de suavidade esférico e o K-funcional}

Nesta seção, introduziremos o módulo de suavidade esférico de uma função e deduziremos uma desigualdade básica envolvendo tal módulo. A dedução de tal propriedade passa pela equivalência entre o módulo de suavidade e o K-funcional de funções de $X$ relativo aos espaços $W_{X}^{r}$. Aparentemente, esses conceitos são de domínio de muitos pesquisadores trabalhando com a Análise na Esfera ou mesmo outras áreas. Entretanto, não encontramos provas autosuficientes para os resultados que precisávamos no contexto do trabalho, ou seja, com a dimensão da esfera fixada mas arbitrária, razão pela qual todas elas estão incluídas aqui.

Módulos de suavidade são extensões naturais dos conhecidos módulos de continuidade. Enquanto estes últimos constituem-se em ferramentas importantes na detecção da suavidade de uma função, os primeiros são úteis na detecção de propriedades mais profundas de suavidade da mesma $([8])$.

Definição 2.8.1. Sejam $f \in X$ e $\delta \in(-1,1)$. O módulo de suavidade esférico é o número dado pela fórmula

$$
w_{1}(\delta, f, X):=\sup _{\delta \leq t<1}\left\|\Delta_{t}(f)\right\|_{X}
$$

Podemos estender o conceito acima, definindo o $r$-ésimo módulo de suavidade, de pelo menos duas maneiras distintas: substituindo-se a diferença $\Delta_{t}$ pela $r$-ésima diferença $\Delta_{t}^{r}$ ou, simplesmente definindo-se

$$
w_{r}(\delta, f, X):=\sup \left\{\left\|\Delta_{t_{1}} \circ \Delta_{t_{2}} \circ \cdots \circ \Delta_{t_{r}}(f)\right\|_{X}: t_{j} \in[\delta, 1), j=1,2, \ldots, r\right\} .
$$


Neste trabalho, não discutiremos a primeira extensão. Mesmo os resultados no restante do capítulo sendo dependentes de $w_{r}(\delta, f, X)$, lembramos o leitor de que, apenas o módulo $w_{1}(\delta, f, X)$ será efetivamente usado nos resultados subseqüentes.

A proposição abaixo enumera as propriedades básicas do módulo de suavidade $w_{r}$.

Proposição 2.8.2. Sejam $f, g \in X$ e $r$ um inteiro positivo. Então valem as seguintes propriedades:

(i) $\lim _{\delta \rightarrow 1^{-}} w_{r}(\delta, f, X)=0$;

(ii) $w_{r}\left(\delta_{2}, f, X\right) \leq w_{r}\left(\delta_{1}, f, X\right),-1<\delta_{1}<\delta_{2}<1$;

(iii) $w_{r}(\delta, f+g, X) \leq w_{r}(\delta, f, X)+w_{r}(\delta, g, X), \delta \in(-1,1)$;

(iv) $w_{r}(\delta, c f, X)=|c| w_{r}(\delta, f, X), c \in \mathbb{R}, \delta \in(-1,1)$;

(v) Se s é um inteiro positivo, então

$$
w_{r+s}(\delta, f, X) \leq 2^{s} w_{r}(\delta, f, X) \leq 2^{r+s}\|f\|_{X}, \quad \delta \in(-1,1)
$$

Demonstração: A propriedade (i) segue da Proposição 2.2.6- $(i)$ enquanto que o item (ii) é óbvio. A propriedade (iii) segue da linearidade dos operadores $\Delta_{t_{j}}$ e da desigualdade triangular. A mesma linearidade justifica o item $(i v)$. Para provar o item $(v)$ sejam $s$ um inteiro positivo e $t_{1}, t_{2}, \ldots, t_{r+s} \in[\delta, 1)$. Pela Proposição 2.2.8- $(i)$, temos que

$$
\begin{aligned}
\left\|\Delta_{t_{1}} \circ \Delta_{t_{2}} \circ \cdots \circ \Delta_{t_{r+s}}(f)\right\|_{X} & \leq 2\left\|\Delta_{t_{2}} \circ \Delta_{t_{3}} \circ \cdots \circ \Delta_{t_{r+s}}(f)\right\|_{X} \\
& \vdots \\
& \leq 2^{s}\left\|\Delta_{t_{s+1}} \circ \Delta_{t_{s+2}} \circ \cdots \circ \Delta_{t_{r+s}}(f)\right\|_{X} \\
& \leq 2^{s} w_{r}(\delta, f, X) .
\end{aligned}
$$

Logo, $w_{r+s}(\delta, f, X) \leq 2^{s} w_{r}(\delta, f, X)$. Além disso,

$$
\begin{aligned}
\left\|\Delta_{t_{1}} \circ \Delta_{t_{2}} \circ \cdots \circ \Delta_{t_{r+s}}(f)\right\|_{X} & \leq 2\left\|\Delta_{t_{2}} \circ \Delta_{t_{3}} \circ \cdots \circ \Delta_{t_{r+s}}(f)\right\|_{X} \\
& \vdots \\
& \leq 2^{s}\left\|\Delta_{t_{s+1}} \circ \Delta_{t_{s+2}} \circ \cdots \circ \Delta_{t_{r+s}}(f)\right\|_{X} \\
& \vdots \\
& \leq 2^{r+s}\|f\|_{X},
\end{aligned}
$$

e, conseqüentemente, $w_{r+s}(\delta, f, X) \leq 2^{r+s}\|f\|_{X}$.

Notemos que os itens $(i i i)$ e $(i v)$ do resultado acima mostram que o módulo $w_{r}$ é uma seminorma em $X$.

A próxima proposição apresenta uma estimativa para o módulo de suavidade em função da derivada forte de Laplace-Beltrami, quando a função reside no espaço $W_{X}^{r}$. 
Proposição 2.8.3. Sejam $r$ um inteiro positivo e $\delta \in(-1,1)$. Então existe uma constante $M(r)$ tal que

$$
w_{r}(\delta, f, X) \leq M(r)(1-\delta)^{r}\left\|D^{r}(f)\right\|_{X}, \quad f \in W_{X}^{r}
$$

Demonstração: Seja $f \in W_{X}^{r}$ e consideremos o caso $r=1$. Usando o Teorema 2.7.10 e a Proposição 2.7.4-(ii), temos que

$$
\left\|\Delta_{t}(f)\right\|_{X}=m\left\|\mathcal{L}_{t}\right\|_{1, m}\left\|D^{1}\left(A_{t}^{1}(f)\right)\right\|_{X}=m\left\|\mathcal{L}_{t}\right\|_{1, m}\left\|A_{t}^{1}\left(D^{1}(f)\right)\right\|_{X}, \quad 0 \leq \delta \leq t<1 .
$$

Pelo Lema 2.5.4 e pela Proposição 1.2.5- $(i)$, vem que

$$
\left\|\Delta_{t}(f)\right\|_{X} \leq m(1-t)\left\|A_{t}^{1}\left(D^{1}(f)\right)\right\|_{X} \leq m(1-t)\left\|G_{t}\right\|_{1, m}\left\|D^{1}(f)\right\|_{X}
$$

Como $\left\|G_{t}\right\|_{1, m}=1$ (veja prova do Lema 2.5.3) concluímos que

$$
\left\|\Delta_{t}(f)\right\|_{X} \leq m(1-t)\left\|D^{1}(f)\right\|_{X} \leq m(1-\delta)\left\|D^{1}(f)\right\|_{X}
$$

Se $-1<\delta \leq 0$ e $\delta \leq t<1$, note inicialmente que o Teorema 2.4 .5 e a definição de $\Delta_{t}$, implicam que

$$
\left\|\Delta_{t}(f)\right\|_{X}=\left\|\Delta_{t}\left(J^{1}\left(D^{1}(f)\right)\right)+\Delta_{t}\left(\mathcal{Y}_{0}(f)\right)\right\|_{X}=\left\|\Delta_{t}\left(J^{1}\left(D^{1}(f)\right)\right)\right\|_{X}
$$

Segue, da Proposição 2.2.8 e da definição de $J^{1}$, que

$$
\left\|\Delta_{t}(f)\right\|_{X} \leq 2\left\|J^{1}\left(D^{1}(f)\right)\right\|_{X}=2\left\|\mathcal{L} * D^{1}(f)\right\|_{X}
$$

Finalmente, pela Proposição 1.2.5,

$$
\left\|\Delta_{t}(f)\right\|_{X} \leq 2\|\mathcal{L}\|_{1, m}\left\|D^{1}(f)\right\|_{X} \leq 2\|\mathcal{L}\|_{1, m}(1-\delta)\left\|D^{1}(f)\right\|_{X}
$$

Tomando-se $M:=\max \left\{m, 2\|\mathcal{L}\|_{1, m}\right\}$ segue que

$$
\left\|\Delta_{t}(f)\right\|_{X} \leq M(1-\delta)\left\|D^{1}(f)\right\|_{X}, \quad \delta \in(-1,1), \quad \delta \leq t<1
$$

Logo,

$$
w_{1}(\delta, f, X) \leq M(1-\delta)\left\|D^{1}(f)\right\|_{X} .
$$

Se $-1<\delta \leq t_{j}<1, j=1,2, \ldots, r$, então usando o que foi feito acima e o Corolário 2.3.11, temos que

$$
\begin{aligned}
\left\|\Delta_{t_{1}} \circ \Delta_{t_{2}} \circ \cdots \circ \Delta_{t_{r}}(f)\right\|_{X} & \leq M(1-\delta)\left\|D^{1}\left(\Delta_{t_{2}} \circ \Delta_{t_{2}} \circ \cdots \circ \Delta_{t_{r}}(f)\right)\right\|_{X} \\
& =M(1-\delta)\left\|\Delta_{t_{2}} \circ \Delta_{t_{2}} \circ \cdots \circ \Delta_{t_{r}}\left(D^{1}(f)\right)\right\|_{X} \\
& \vdots \\
& \leq M^{r}(1-\delta)^{r}\left\|D^{r}(f)\right\|_{X} .
\end{aligned}
$$


Definindo-se $M(r):=M^{r}$ segue que

$$
w_{r}(\delta, f, X) \leq M(r)(1-\delta)^{r}\left\|D^{r}(f)\right\|_{X}
$$

o que conclui a prova.

A propriedade abaixo não é utilizada no restante do trabalho, mas com o intuito de deixar o trabalho mais completo optamos por colocá-la. Ela compara o $r$-ésimo módulo de suavidade esférico com o $s$-ésimo módulo de suavidade esférico quando $s<r$.

Proposição 2.8.4. Sejam $\delta \in(-1,1)$ e $r$, s inteiros positivos tais que $s<r$. Então existe uma constante $M(q)$ tal que

$$
w_{r}(\delta, f, X) \leq M(r-s)(1-\delta)^{r-s} w_{s}\left(\delta, D^{r-s}(f), X\right), \quad f \in W_{X}^{r-s}
$$

Demonstração: Sejam $q=r-s$ e $f \in W_{X}^{q}$. Se $-1<\delta \leq t_{j}<1, j=1,2, \ldots, r$, usamos o que foi feito na primeira parte da demonstração da proposição anterior e o Corolário 2.3.11 para obter

$$
\begin{aligned}
\left\|\Delta_{t_{1}} \circ \Delta_{t_{2}} \circ \cdots \circ \Delta_{t_{r}}(f)\right\|_{X} & \leq M(1-\delta) \| D^{1}\left(\Delta_{t_{2}} \circ \Delta_{t_{3}} \circ \cdots \circ \Delta_{t_{r}}(f) \|_{X}\right. \\
& =M(1-\delta)\left\|\Delta_{t_{2}} \circ \Delta_{t_{3}} \circ \cdots \circ \Delta_{t_{r}}\left(D^{1}(f)\right)\right\|_{X} \\
& \vdots \\
& \leq M^{q}(1-\delta)^{q}\left\|\Delta_{t_{q+1}} \circ \Delta_{t_{q+2}} \circ \cdots \circ \Delta_{t_{r}}\left(D^{q}(f)\right)\right\|_{X} \\
& \leq M^{q}(1-\delta)^{q} w_{r-q}\left(\delta, D^{q}(f), X\right),
\end{aligned}
$$

onde $M$ é uma constante positiva. Logo,

$$
w_{r}(\delta, f, X) \leq M(q)(1-\delta)^{q} w_{r-q}\left(\delta, D^{q}(f), X\right)
$$

onde $M(q):=M^{q}$, e a proposição está provada.

A constante $M(r)$ da Proposição 2.8.3 pode ser melhorada, se $\delta$ estiver suficientemente próximo de 1 . A veracidade deste fato pode ser constatada na proposição abaixo.

Proposição 2.8.5. Se r é um inteiro positivo, existe $\bar{\delta} \in(-1,1)$ tal que se $\delta \in[\bar{\delta}, 1)$, então

$$
w_{r}(\delta, f, X) \leq 2^{r}(1-\delta)^{r}\left\|D^{r}(f)\right\|_{X}, \quad f \in W_{X}^{r}
$$

Demonstração: Seja $f \in W_{X}^{1}$. Obviamente

$$
\begin{aligned}
\left\|\Delta_{t}(f)\right\|_{X} & \leq(1-t)\left\|\frac{\Delta_{t}(f)}{1-t}-D^{1}(f)+D^{1}(f)\right\|_{X} \\
& \leq(1-t)\left(\left\|\frac{\Delta_{t}(f)}{1-t}-D^{1}(f)\right\|_{X}+\left\|D^{1}(f)\right\|_{X}\right), \quad t \in(-1,1) .
\end{aligned}
$$


Da definição de $D^{1}(f)$, existe $0<\delta^{\prime}<2$ tal que, se $1-t<\delta^{\prime}$, então

$$
\left\|\frac{\Delta_{t}(f)}{1-t}-D^{1}(f)\right\|_{X}<\left\|D^{1}(f)\right\|_{X} .
$$

Logo, tomando-se $\delta>1-\delta^{\prime}$, segue que

$$
\begin{aligned}
w_{1}(\delta, f, X) & \leq \sup _{\delta \leq t<1}(1-t)\left(\left\|\frac{\Delta_{t}(f)}{1-t}-D^{1}(f)\right\|_{X}+\left\|D^{1}(f)\right\|_{X}\right) \\
& =(1-\delta) \sup _{\delta \leq t<1}\left\|\frac{\Delta_{t}(f)}{1-t}-D^{1}(f)\right\|_{X}+(1-\delta)\left\|D^{1}(f)\right\|_{X} \\
& \leq 2(1-\delta)\left\|D^{1}(f)\right\|_{X} .
\end{aligned}
$$

Escrevendo $1-\delta^{\prime}=\bar{\delta}$, o resultado segue neste caso. No caso geral utilizamos um raciocínio análogo àquele usado na prova da Proposição 2.8.3.

A seguir, introduziremos o $K$-funcional de uma função de $X$ relativo ao espaço $W_{X}^{r}$. Assim como o módulo de suavidade esférico, o $K$-funcional fornece informações sobre a suavidade da função dada.

Definição 2.8.6. Sejam $r$ um inteiro positivo e $t>0$. $O K$-funcional de $f \in X$ relativo a $W_{X}^{r}$ é o número real não negativo dado por

$$
K\left(t, f, X, W_{X}^{r}\right):=\inf \left\{\|f-g\|_{X}+t\left\|D^{r}(g)\right\|_{X}: g \in W_{X}^{r}\right\} .
$$

Proposição 2.8.7. Sejam $r$ e s inteiros positivos, $f \in X$ et $>0$. Então

$$
K\left(s t, f, X, W_{X}^{r}\right) \leq \max \{1, t\} K\left(s, f, X, W_{X}^{r}\right) \leq(1+t) K\left(s, f, X, W_{X}^{r}\right) .
$$

Demonstração: Suponhamos primeiramente que $0<t \leq 1$. Então $\max \{1, t\}=1$ e $t s \leq s$. Daí, segue que

$$
\|f-g\|_{X}+t s\left\|D^{r}(g)\right\|_{X} \leq\|f-g\|_{X}+s\left\|D^{r}(g)\right\|_{X}, \quad g \in W_{X}^{r} .
$$

Logo,

$$
\inf \left\{\|f-g\|_{X}+t s\left\|D^{r}(g)\right\|_{X}: g \in W_{X}^{r}\right\} \leq \inf \left\{\|f-g\|_{X}+s\left\|D^{r}(g)\right\|_{X}: g \in W_{X}^{r}\right\},
$$

e, conseqüentemente,

$$
K\left(s t, f, X, W_{X}^{r}\right) \leq \max \{1, t\} K\left(s, f, X, W_{X}^{r}\right) \leq(1+t) K\left(s, f, X, W_{X}^{r}\right) .
$$

Suponhamos agora que $t>1$. Então $\max \{1, t\}=t \mathrm{e}$

$$
\|f-g\|_{X}+t s\left\|D^{r}(g)\right\|_{X} \leq t\left(\|f-g\|_{X}+s\left\|D^{r}(g)\right\|_{X}\right), \quad g \in W_{X}^{r} .
$$

Logo,

$$
\inf \left\{\|f-g\|_{X}+t s\left\|D^{r}(g)\right\|_{X}: g \in W_{X}^{r}\right\} \leq t \inf \left\{\|f-g\|_{X}+s\left\|D^{r}(g)\right\|_{X}: g \in W_{X}^{r}\right\},
$$

e, portanto,

$$
K\left(s t, f, X, W_{X}^{r}\right) \leq \max \{1, t\} K\left(s, f, X, W_{X}^{r}\right) \leq(1+t) K\left(s, f, X, W_{X}^{r}\right) .
$$

Isso conclui a prova da proposição. 


\subsection{Equivalência entre o módulo de suavidade e o $K$ - funcional}

Nesta seção provaremos a equivalência entre o $K$-funcional e o módulo de suavidade esférico. A equivalência produz uma propriedade adicional do módulo de suavidade esférico, a ser usada na análise da ordem de convergência dos processos de aproximação discutidos no trabalho.

A equivalência propriamente dita é descrita no teorema a seguir.

Teorema 2.9.1. Sejam $r$ um inteiro positivo e $\delta \in(-1,1)$. Então existem constantes $m_{r}$ e $M_{r}$ tais que

$$
m_{r} w_{r}(\delta, f, X) \leq K\left((1-\delta)^{r}, f, X, W_{X}^{r}\right) \leq M_{r} w_{r}(\delta, f, X), \quad f \in X
$$

Demonstração: Seja $f \in X$. Usando a Proposição 2.8.2-(iii),(v) e a Proposição 2.8.3, vemos que

$$
\begin{aligned}
w_{r}(\delta, f, X) & \leq w_{r}(\delta, f-g, X)+w_{r}(\delta, g, X) \\
& \leq 2^{r}\|f-g\|_{X}+M(r)(1-\delta)^{r}\left\|D^{r}(g)\right\|_{X}, \quad g \in W_{X}^{r},
\end{aligned}
$$

onde $M(r)$ é a constante definida na Proposição 2.8.3. Definindo-se $m_{r}^{-1}:=\max \left\{2^{r}, M(r)\right\}$, vem que

$$
w_{r}(\delta, f, X) \leq m_{r}^{-1}\left(\|f-g\|_{X}+(1-\delta)^{r}\left\|D^{r}(g)\right\|_{X}\right), \quad g \in W_{X}^{r}
$$

Logo,

$$
m_{r} w_{r}(\delta, f, X) \leq K\left((1-\delta)^{r}, f, X, W_{X}^{r}\right)
$$

Para obter a outra desigualdade do teorema, vamos estimar $K\left((1-\delta)^{r}, f, X, W_{X}^{r}\right)$ usando uma função particular de $W_{X}^{r}$. Para tanto, definamos (veja Definição 2.6.1)

$$
B_{\delta}^{r}(f):=\sum_{j=1}^{r}(-1)^{j+1}\left(\begin{array}{l}
r \\
j
\end{array}\right) A_{\delta}^{r j}(f) .
$$

Então, $B_{\delta}^{r}(f)=f-\left(I-A_{\delta}^{r}\right)^{r}(f)$, onde $I$ é o operador identidade. Como $A_{\delta}^{r}(f) \in W_{X}^{r}$ segue que $B_{\delta}^{r}(f) \in W_{X}^{r}$. Das Proposições 2.7.4 e 1.2.5 vem que

$$
\begin{aligned}
\left\|D^{r}\left(A_{\delta}^{r j}(f)\right)\right\|_{X} & =\left\|A_{\delta}^{r j}\left(D^{r}(f)\right)\right\|_{X} \\
& \leq\left\|G_{\delta}\right\|_{1, m}^{(j-1) r}\left\|D^{r}\left(A_{\delta}^{r}(f)\right)\right\|_{X} \\
& =\left\|D^{r}\left(A_{\delta}^{r}(f)\right)\right\|_{X}, \quad j=1,2, \ldots, r
\end{aligned}
$$


enquanto que a linearidade de $D^{r}$ implica que

$$
\begin{aligned}
\left\|D^{r}\left(B_{\delta}^{r}(f)\right)\right\|_{X} & =\left\|\sum_{j=1}^{r}(-1)^{j+1}\left(\begin{array}{l}
r \\
j
\end{array}\right) D^{r}\left(A_{\delta}^{r j}(f)\right)\right\|_{X} \\
& \leq \sum_{j=1}^{r}\left(\begin{array}{l}
r \\
j
\end{array}\right)\left\|D^{r}\left(A_{\delta}^{r j}(f)\right)\right\|_{X} \\
& \leq\left\|D^{r}\left(A_{\delta}^{r}(f)\right)\right\|_{X} \sum_{j=1}^{r}\left(\begin{array}{l}
r \\
j
\end{array}\right) \\
& =\left(2^{r}-1\right)\left\|D^{r}\left(A_{\delta}^{r}(f)\right)\right\|_{X} .
\end{aligned}
$$

Logo, usando o Teorema 2.7.10-(ii) e o Lema 2.5.5 temos que

$$
\begin{aligned}
\left\|D^{r}\left(B_{\delta}^{r}(f)\right)\right\|_{X} & \leq\left(2^{r}-1\right) m^{-r}\left\|\mathcal{L}_{h}\right\|_{1, m}^{-r}\left\|\Delta_{\delta}^{r}(f)\right\|_{X} \\
& \leq\left(2^{r}-1\right)(1-\delta)^{-r}\left\|\Delta_{\delta}^{r}(f)\right\|_{X} .
\end{aligned}
$$

Passando à estimativa propriamente dita, notemos inicialmente que o Lema 2.6.2 implica que

$$
\left\|f-B_{\delta}^{1}(f)\right\|_{X}=\left\|\left(I-A_{\delta}^{1}\right)(f)\right\|_{X} \leq \sup \left\{\left\|\Delta_{t}(f)\right\|_{X}: t \in[\delta, 1)\right\}=w_{1}(\delta, f, X) .
$$

Se $r=2$, então usando novamente o Lema 2.6.2, deduzimos

$$
\begin{aligned}
\left\|f-B_{\delta}^{2}(f)\right\|_{X} & =\left\|\left(I-A_{\delta}^{2}\right)^{2}(f)\right\|_{X} \\
& =\left\|\left(I-A_{\delta}^{2}\right) \circ\left(I-A_{\delta}^{2}\right)(f)\right\|_{X} \\
& \leq 2 \sup \left\{\left\|\Delta_{t_{2}} \circ\left(I-A_{\delta}^{2}\right)(f)\right\|_{X}: t_{2} \in[\delta, 1)\right\} .
\end{aligned}
$$

Como $\Delta_{t_{2}}$ e $\left(I-A_{\delta}^{2}\right)$ comutam (Lema 2.6.4), temos que

$$
\begin{aligned}
\left\|f-B_{\delta}^{2}(f)\right\|_{X} & \leq 2 \sup \left\{\left\|\left(I-A_{\delta}^{2}\right) \circ \Delta_{t_{2}}(f)\right\|_{X}: t_{2} \in[\delta, 1)\right\} \\
& \leq 4 \sup \left\{\left\|\Delta_{t_{1}} \circ \Delta_{t_{2}}(f)\right\|_{X}: t_{1}, t_{2} \in[\delta, 1)\right\} \\
& =4 w_{2}(\delta, f, X) .
\end{aligned}
$$

Em geral, deduzimos que

$$
\left\|f-B_{\delta}^{r}(f)\right\|_{X} \leq r^{r} w_{r}(\delta, f, X)
$$

Assim,

$$
\begin{aligned}
K\left((1-\delta)^{r}, f, X, W_{X}^{r}\right) & \leq\left\|f-B_{\delta}^{r}(f)\right\|_{X}+(1-\delta)^{r}\left\|D^{r}\left(B_{\delta}^{r}(f)\right)\right\|_{X} \\
& \leq r^{r} w_{r}(\delta, f, X)+\left(2^{r}-1\right)\left\|\Delta_{\delta}^{r}(f)\right\|_{X} \\
& \leq r^{r} w_{r}(\delta, f, X)+\left(2^{r}-1\right) w_{r}(\delta, f, X) \\
& \leq M_{r} w_{r}(\delta, f, X),
\end{aligned}
$$

onde $M_{r}:=\max \left\{r^{r}, 2^{r}-1\right\}$. Isso completa a prova do teorema. 
Corolário 2.9.2. Sejam $f \in X$ e r um inteiro positivo. Então $\lim _{t \rightarrow 0} K\left(t, f, X, W_{X}^{r}\right)=0$.

Demonstração: Segue diretamente do teorema anterior e da Proposição 2.8.2-(i).

Finalizamos a seção apresentando um corolário de Teorema 2.9.1 que será usado no Capítulo 4 .

Corolário 2.9.3. Sejam $r$ um inteiro positivo e $\delta_{1}, \delta_{2} \in(-1,1)$. Então existe uma constante $C(r)$ tal que

$$
w_{r}\left(\delta_{1}, f, X\right) \leq C(r)\left[1+\left(\frac{1-\delta_{1}}{1-\delta_{2}}\right)^{r}\right] w_{r}\left(\delta_{2}, f, X\right), \quad f \in X
$$

Demonstração: Seja $f \in X$. Usando a primeira desigualdade do Teorema 2.9.1 e a Proposição 2.8.7, segue que

$$
\begin{aligned}
w_{r}\left(\delta_{1}, f, X\right) & \leq \frac{1}{m_{r}} K\left(\left(1-\delta_{1}\right)^{r}, f, X, W_{X}^{r}\right) \\
& =\frac{1}{m_{r}} K\left(\left(\frac{1-\delta_{1}}{1-\delta_{2}}\right)^{r}\left(1-\delta_{2}\right)^{r}, f, X, W_{X}^{r}\right) \\
& \leq \frac{1}{m_{r}} \max \left\{1,\left(\frac{1-\delta_{1}}{1-\delta_{2}}\right)^{r}\right\} K\left(\left(1-\delta_{2}\right)^{r}, f, X, W_{X}^{r}\right),
\end{aligned}
$$

enquanto que a segunda desigualdade do teorema anterior nos leva a

$$
\begin{aligned}
w_{r}\left(\delta_{1}, f, X\right) & \leq \frac{M_{r}}{m_{r}} \max \left\{1,\left(\frac{1-\delta_{1}}{1-\delta_{2}}\right)^{r}\right\} w_{r}\left(\delta_{2}, f, X\right) \\
& \leq C(r)\left[1+\left(\frac{1-\delta_{1}}{1-\delta_{2}}\right)^{r}\right] w_{r}\left(\delta_{2}, f, X\right),
\end{aligned}
$$

onde $C(r):=M_{r} / m_{r}$. 


\section{Capítulo}

3

\section{Aproximação por somas com pesos de harmônicos esféricos}

Neste capítulo, detalharemos aquilo que chamamos de aproximação por somas com pesos de harmônicos esféricos. Introduziremos os operadores de aproximação relativos aos processos em questão e discutiremos várias propriedades desses operadores, incluindo o cálculo de suas normas em alguns casos. Concluiremos o capítulo, analisando as propriedades aproximatórias propriamente ditas, principalmente aquelas referentes à obtenção de aproximações da identidade em $X$ a partir dos operadores.

\subsection{O operador $T_{n}$}

Fixadas bases para os espaços $\mathcal{H}_{k}\left(S^{m}\right)$ como no primeiro capítulo, investigaremos processos aproximatórios definidos por operadores $T_{n}: X \rightarrow X, n=0,1, \ldots$, da forma

$$
T_{n}(f)=\sum_{k=0}^{n} \sum_{l=1}^{N(m, k)} a_{k l}(n) \hat{f}(k, l) Y_{k l}, \quad f \in X
$$

onde $a_{k l}(n) \in \mathbb{R}, n, k=0,1, \ldots, l=1,2, \ldots, N(m, k)$. Notamos que cada $T_{n}$ é uma expansão de Fourier truncada que inclui harmônicos esféricos até grau $n$. Os pesos a que se refere o título do trabalho e do capítulo são, então, os números reais $a_{k l}(n)$. A ação de $T_{n}(f)$ em um ponto $x \in S^{m}$ será denotada por $T_{n} f(x):=\left(T_{n}(f)\right)(x)$ em várias passagens do texto.

A ortonormalidade dos harmônicos esféricos implica imediatamente que

$$
T_{n}\left(Y_{\mu \nu}\right)= \begin{cases}a_{\mu \nu}(n) Y_{\mu \nu}, & \mu \leq n \\ 0, & \mu>n,\end{cases}
$$

enquanto que a linearidade de $T_{n}$ revela que se $q=\sum_{\mu=0}^{M} \sum_{\nu=1}^{N(m, \mu)} r_{\mu \nu} Y_{\mu \nu}$ é um polinômio arbitrário de grau $M$ em $\mathbb{P}_{n}\left(S^{m}\right)$, então

$$
T_{n}(q)=\sum_{k=0}^{M} \sum_{l=1}^{N(m, k)} a_{k l}(n) r_{k l} Y_{k l}, \quad n \geq M .
$$


Utilizando-se a definição do coeficiente de Fourier na definição de $T_{n}$ obtemos:

$$
\begin{aligned}
T_{n} f(x) & =\frac{1}{\sigma_{m}} \sum_{k=0}^{n} \sum_{l=1}^{N(m, k)} a_{k l}(n)\left(\int_{S^{m}} f(y) \overline{Y_{k l}(y)} d \sigma_{m}(y)\right) Y_{k l}(x) \\
& =\frac{1}{\sigma_{m}} \int_{S^{m}} f(y) \sum_{k=0}^{n} \sum_{l=1}^{N(m, k)} a_{k l}(n) Y_{k l}(x) \overline{Y_{k l}(y)} d \sigma_{m}(y)
\end{aligned}
$$

Logo, provamos o seguinte teorema.

Teorema 3.1.1. Se $f \in X$, então

$$
T_{n} f(x)=\frac{1}{\sigma_{m}} \int_{S^{m}} K_{n}(x, y) f(y) d \sigma_{m}(y), \quad x \in S^{m},
$$

onde

$$
K_{n}(x, y)=\sum_{k=0}^{n} \sum_{l=1}^{N(m, k)} a_{k l}(n) Y_{k l}(x) \overline{Y_{k l}(y)}, \quad x, y \in S^{m} .
$$

No restante desta seção utilizaremos a representação descrita acima para estabelecer uma conexão entre $T_{n}$ e o conceito de convolução esférica. Isto de certa forma é uma das facetas que motivaram o estudo de aproximações usando a seqüência $\left\{T_{n}\right\}_{n \in \mathbb{N}}$.

Núcleos que dependem do produto interno entre as variáveis, isto é, núcleos da forma $K(x, y)=L(\langle x, y\rangle), x, y \in S^{m}$, para alguma função $L$, são chamados núcleos bi-zonais. As proposições abaixo analisam a possibilidade de $T_{n}$ ser um operador de convolução.

Proposição 3.1.2. O núcleo $K_{n}$ é bi-zonal se, e somente se, $a_{k 1}(n)=a_{k 2}(n)=\cdots=$ $a_{k N(m, k)}(n), k \in \mathbb{N}$.

Demonstração: Se $a_{k 1}(n)=a_{k 2}(n)=\cdots=a_{k N(m, k)}(n), k \in \mathbb{N}$, podemos usar o Teorema da Adição para obter

$$
\begin{aligned}
K_{n}(x, y) & =\sum_{k=0}^{n} a_{k 1}(n) \sum_{l=1}^{N(m, k)} Y_{k l}(x) \overline{Y_{k l}(y)} \\
& =\sum_{k=0}^{n} a_{k 1}(n) N(m, k) P_{k}^{m}(\langle x, y\rangle):=L_{n}(\langle x, y\rangle), \quad x, y \in S^{m} .
\end{aligned}
$$

Logo, $K_{n}$ é bi-zonal. Reciprocamente, se $K_{n}(x, y)=L_{n}(\langle x, y\rangle)$ para algum $L_{n}$, então a Fórmula de Funk-Hecke nos dá

$$
\begin{aligned}
\int_{S^{m}} K_{n}(x, y) Y_{k l}(y) d \sigma_{m}(y) & =\int_{S^{m}} L_{n}(\langle x, y\rangle) Y_{k l}(y) d \sigma_{m}(y) \\
& =a_{k}^{m}\left(L_{n}\right) Y_{k l}(x), \quad x \in S^{m},
\end{aligned}
$$

enquanto que o teorema anterior e a Equação (3.2) implicam que

$$
\int_{S^{m}} K_{n}(x, y) Y_{k l}(y) d \sigma_{m}(y)=\sigma_{m} a_{k l}(n) Y_{k l}(x), \quad x \in S^{m}, \quad k \leq n .
$$


Logo,

$$
a_{k}^{m}\left(L_{n}\right) Y_{k l}(x)=\sigma_{m} a_{k l}(n) Y_{k l}(x), \quad x \in S^{m} .
$$

Como $Y_{k l} \not \equiv 0$, segue que $a_{k l}(n)=a_{k}^{m}\left(L_{n}\right) / \sigma_{m}, l=1,2, \ldots, N(m, k)$.

Proposição 3.1.3. O operador $T_{n}$ é um operador de convolução se, e somente se, o núcleo $K_{n}$ é bi-zonal.

Demonstração: Se $K_{n}(x, y)=L_{n}(\langle x, y\rangle), x, y \in S^{m}$, para alguma função $L_{n}$, então a representação do Teorema 3.1.1 implica que $T_{n}(f)=L_{n} * f, f \in X$. Por outro lado, se $T_{n}(f)=L_{n} * f, f \in X$, para alguma função $L_{n}$, então

$$
\int_{S^{m}}\left[K_{n}(x, y)-L_{n}(\langle x, y\rangle)\right] f(y) d \sigma_{m}(y)=0, \quad x \in S^{m}, \quad f \in X .
$$

Em particular, se $k \in \mathbb{N}$ e $l=1,2, \ldots, N(m, k)$, então

$$
\int_{S^{m}}\left[K_{n}(x, y)-L_{n}(\langle x, y\rangle)\right] Y_{k l}(y) d \sigma_{m}(y)=0, \quad x \in S^{m}
$$

Como $\left\{Y_{k l}: k \in \mathbb{N}, l=1,2, \ldots, N(m, k)\right\}$ é um conjunto ortonormal completo de $L^{2}\left(S^{m}\right)$, segue que

$$
K_{n}(x, y)-L_{n}(\langle x, y\rangle)=0 \quad x, y \in S^{m}, \quad \text { q.s.. }
$$

Como $K_{n}$ é polinomial, segue que $K_{n}(x, y)=L_{n}(\langle x, y\rangle), x, y \in S^{m}$.

\subsection{A norma de $T_{n}$}

Nesta seção, calcularemos a norma do operador $T_{n}$. A norma desse operador será denotada por $\left\|T_{n}\right\|_{X}$ apesar dessa notação ser semelhante àquela adotada no Capítulo 1.

Se $K: S^{m} \times S^{m} \rightarrow \mathbb{C}$ é um núcleo qualquer, escreveremos $K^{x}$ e $K^{y}$ para denotar as funções $y \in S^{m} \mapsto K(x, y)$ e $x \in S^{m} \mapsto K(x, y)$, respectivamente. Observamos que se $K$ é polinomial, então ambas as funções $K^{x}$ e $K^{y}$ são elementos de $L^{1}\left(S^{m}\right)$. Em um passo da prova do teorema abaixo usamos o espaço $L^{\infty}\left(S^{m}\right)$ como definido em ([10], p. 184).

Teorema 3.2.1. Vale a seguinte desigualdade: $\left\|T_{n}\right\|_{X} \leq \sup \left\{\left\|K_{n}^{x}\right\|_{1}: x \in S^{m}\right\}$.

Demonstração: Vejamos inicialmente o caso em que $X=C\left(S^{m}\right)$. Se $f \in C\left(S^{m}\right)$, a representação dada no Teorema 3.1.1 implica que

$$
\left|T_{n} f(x)\right| \leq \frac{1}{\sigma_{m}} \int_{S^{m}}\left|K_{n}(x, y) f(y)\right| d \sigma_{m}(y) \leq\left\|K_{n}^{x}\right\|_{1}\|f\|_{\infty}, \quad x \in S^{m}
$$

Logo,

$$
\left\|T_{n}(f)\right\|_{\infty} \leq\|f\|_{\infty} \sup \left\{\left\|K_{n}^{x}\right\|_{1}: x \in S^{m}\right\}, \quad f \in C\left(S^{m}\right),
$$


e, conseqüentemente,

$$
\left\|T_{n}\right\|_{C\left(S^{m}\right)} \leq \sup \left\{\left\|K_{n}^{x}\right\|_{1}: x \in S^{m}\right\} .
$$

Para os demais casos, sejam $f \in L^{p}\left(S^{m}\right)$ e $p^{\prime}$ o expoente conjugado de $p$. Então

$$
\begin{aligned}
\left\|T_{n}(f)\right\|_{p}^{p} & =\frac{1}{\sigma_{m}} \int_{S^{m}}\left|T_{n} f(x)\right|^{p} d \sigma_{m}(x) \\
& =\frac{1}{\sigma_{m}} \int_{S^{m}}\left|\frac{1}{\sigma_{m}} \int_{S^{m}} K_{n}(x, y) f(y) d \sigma_{m}(y)\right|^{p} d \sigma_{m}(x) \\
& \leq \frac{1}{\sigma_{m}} \int_{S^{m}}\left[\frac{1}{\sigma_{m}} \int_{S^{m}}\left|K_{n}(x, y) f(y)\right| d \sigma_{m}(y)\right]^{p} d \sigma_{m}(x) .
\end{aligned}
$$

Como $\left(K_{n}^{x}\right)^{1 / p} f \in L^{p}\left(S^{m}\right), x \in S^{m}$ e $\left(K_{n}^{x}\right)^{1 / p^{\prime}} \in L^{p^{\prime}}\left(S^{m}\right), x \in S^{m}$, a desigualdade de Hölder implica que

$$
\begin{aligned}
{\left[\frac{1}{\sigma_{m}} \int_{S^{m}}\left|K_{n}(x, y) f(y)\right| d \sigma_{m}(y)\right]^{p} } & \leq\left\|\left(K_{n}^{x}\right)^{1 / p^{\prime}}\right\|_{p^{\prime}}^{p}\left\|\left(K_{n}^{x}\right)^{1 / p} f\right\|_{p}^{p} \\
& =\left\|K_{n}^{x}\right\|_{1}^{p / p^{\prime}} \frac{1}{\sigma_{m}} \int_{S^{m}}\left|K_{n}(x, y) \| f(y)\right|^{p} d \sigma_{m}(y) .
\end{aligned}
$$

Como a função $x \in S^{m} \mapsto\left\|K_{n}^{x}\right\|_{1} \in \mathbb{R}$ é contínua e $S^{m}$ é compacta, existe um ponto $x_{0} \in S^{m}$ tal que

$$
\sup \left\{\left\|K_{n}^{x}\right\|_{1}: x \in S^{m}\right\}=\left\|K_{n}^{x_{0}}\right\|_{1} .
$$

Logo, usando o Teorema de Fubini ([33], p. 384), segue que

$$
\begin{aligned}
\left\|T_{n} f\right\|_{p}^{p} & \leq \frac{1}{\sigma_{m}} \int_{S^{m}}\left\|K_{n}^{x}\right\|_{1}^{p / p^{\prime}} \frac{1}{\sigma_{m}} \int_{S^{m}}\left|K_{n}(x, y) \| f(y)\right|^{p} d \sigma_{m}(y) d \sigma_{m}(x) \\
& \leq\left\|K_{n}^{x_{0}}\right\|_{1}^{p / p^{\prime}} \frac{1}{\sigma_{m}} \int_{S^{m}} \frac{1}{\sigma_{m}} \int_{S^{m}}\left|K_{n}(x, y)\right||f(y)|^{p} d \sigma_{m}(y) d \sigma_{m}(x) \\
& =\left\|K_{n}^{x_{0}}\right\|_{1}^{p / p^{\prime}} \frac{1}{\sigma_{m}} \int_{S^{m}}\left(\frac{1}{\sigma_{m}} \int_{S^{m}}\left|\overline{K_{n}(y, x)}\right| d \sigma_{m}(x)\right)|f(y)|^{p} d \sigma_{m}(y),
\end{aligned}
$$

ou seja,

$$
\begin{aligned}
\left\|T_{n} f\right\|_{p}^{p} & \leq\left\|K_{n}^{x_{0}}\right\|_{1}^{p / p^{\prime}} \frac{1}{\sigma_{m}} \int_{S^{m}}\left\|K_{n}^{y}\right\|_{1}|f(y)|^{p} d \sigma_{m}(y) \\
& \leq\left\|K_{n}^{x_{0}}\right\|_{1}^{p / p^{\prime}}\left\|K_{n}^{x_{0}}\right\|_{1}\|f\|_{p}^{p} \\
& =\left\|K_{n}^{x_{0}}\right\|_{1}\|f\|_{p}^{p} .
\end{aligned}
$$

Segue que

$$
\left\|T_{n} f\right\|_{p} \leq \sup \left\{\left\|K_{n}^{x}\right\|_{1}: x \in S^{m}\right\}\|f\|_{p},
$$

e, portanto,

$$
\left\|T_{n}\right\|_{L^{p}\left(S^{m}\right)} \leq \sup \left\{\left\|K_{n}^{x}\right\|_{1}: x \in S^{m}\right\}
$$

Isso conclui a prova do teorema. 
Na prova do próximo resultado, utilizaremos as seguintes fórmulas para calcular a norma de um elemento de $C\left(S^{m}\right)$ ou $L_{1}\left(S^{m}\right)$. Se $f \in C\left(S^{m}\right)$, então

$$
\|f\|_{\infty}=\sup \left\{\left|\frac{1}{\sigma_{m}} \int_{S^{m}} f \bar{g} d \sigma_{m}\right|:\|g\|_{1}=1\right\} .
$$

Se $f \in L^{1}\left(S^{m}\right)$, então

$$
\|f\|_{1}=\sup \left\{\left|\frac{1}{\sigma_{m}} \int_{S^{m}} f \bar{g} d \sigma_{m}\right|:\|g\|_{\infty}=1\right\} .
$$

A primeira fórmula pode ser obtida do Teorema de Representação de Riesz para $C\left(S^{m}\right)$ ([10], p. 216). Na verdade, se a função $f$ é não-negativa, então ela segue do Teorema 7.2 de ([10] p. 205). A outra fórmula pode ser obtida da Desigualdade Inversa de Hölder ([10], p. 181).

Teorema 3.2.2. Se $X=C\left(S^{m}\right)$ ou $X=L^{1}\left(S^{m}\right)$, então

$$
\left\|T_{n}\right\|_{X}=\sup \left\{\left\|K_{n}^{x}\right\|_{1}: x \in S^{m}\right\}
$$

Demonstração: Em vista do teorema anterior basta provar que

$$
\left\|T_{n}\right\|_{X} \geq \sup \left\{\left\|K_{n}^{x}\right\|_{1}: x \in S^{m}\right\} .
$$

Por um lado temos que

$$
\begin{aligned}
\left\|K_{n}^{x}\right\|_{1} & =\sup \left\{\left|\frac{1}{\sigma_{m}} \int_{S^{m}} K_{n}(x, y) \overline{f(y)} d \sigma_{m}(y)\right|:\|f\|_{\infty}=1\right\} \\
& =\sup \left\{\left|\frac{1}{\sigma_{m}} \int_{S^{m}} K_{n}(x, y) f(y) d \sigma_{m}(y)\right|:\|\bar{f}\|_{\infty}=1\right\} \\
& =\sup \left\{\left|T_{n} f(x)\right|:\|f\|_{\infty}=1\right\} \\
& \leq \sup \left\{\left\|T_{n}(f)\right\|_{\infty}:\|f\|_{\infty}=1\right\} \\
& =\left\|T_{n}\right\|_{C\left(S^{m}\right)}, \quad x \in S^{m} .
\end{aligned}
$$

Logo,

$$
\sup \left\{\left\|K_{n}^{x}\right\|_{1}: x \in S^{m}\right\} \leq\left\|T_{n}\right\|_{C\left(S^{m}\right)} .
$$

Por outro lado, temos que

$$
\begin{aligned}
\int_{S^{m}} T_{n} f(y) \overline{g(y)} d \sigma_{m}(y) & =\int_{S^{m}} \sum_{k=0}^{n} \sum_{l=1}^{N(m, k)} a_{k l}(n) \hat{f}(k, l) Y_{k l}(y) \overline{g(y)} d \sigma_{m}(y) \\
& =\int_{S^{m}}\left(\sum_{k=0}^{n} \sum_{l=1}^{N(m, k)} a_{k l}(n) \overline{\hat{g}(k, l)} \overline{Y_{k l}(y)}\right) f(y) d \sigma_{m}(y) \\
& =\overline{\int_{S^{m}} T_{n} g(y) \overline{f(y)} d \sigma_{m}(y)}
\end{aligned}
$$


Logo,

$$
\begin{aligned}
\left\|T_{n}\right\|_{L_{1}\left(S^{m}\right)} & =\sup \left\{\left\|T_{n} f\right\|_{1}:\|f\|_{1}=1\right\} \\
& =\sup _{\|f\|_{1}=1}\left(\sup \left\{\left|\frac{1}{\sigma_{m}} \int_{S^{m}} T_{n} f(y) \overline{g(y)} d \sigma_{m}(y)\right|:\|g\|_{\infty}=1\right\}\right) \\
& =\sup _{\|f\|_{1}=1}\left(\sup \left\{\left|\frac{1}{\sigma_{m}} \int_{S^{m}} T_{n} g(y) \overline{f(y)} d \sigma_{m}(y)\right|:\|g\|_{\infty}=1\right\}\right) \\
& =\sup _{\|g\|_{\infty}=1}\left(\sup \left\{\left|\frac{1}{\sigma_{m}} \int_{S^{m}} T_{n} g(y) \overline{f(y)} d \sigma_{m}(y)\right|:\|f\|_{1}=1\right\}\right) \\
& =\sup \left\{\left\|T_{n}(g)\right\|_{\infty}:\|g\|_{\infty}=1\right\} \\
& =\left\|T_{n}\right\|_{C\left(S^{m}\right)} .
\end{aligned}
$$

O teorema segue.

Após algumas tentativas não conseguimos obter uma versão do teorema acima no caso em que $X=L^{p}\left(S^{m}\right), p>1$. Entretanto, acreditamos na existência de tal versão.

\subsection{Aproximações da identidade}

Nos resultados a seguir, investigaremos a seguinte propriedade de convergência:

$$
\lim _{n \rightarrow \infty}\left\|T_{n}(f)-f\right\|_{X}=0, \quad f \in X
$$

Lembramos que se tal fenômeno ocorre, a seqüência $\left\{T_{n}\right\}_{n \in \mathbb{N}}$ é denominada uma aproximação da identidade em $X$. Entretanto oservamos que esta mesma terminologia é também usada em situações mais refinadas. Alguns exemplos de aproximações da identidade em $X$, incluindo exemplos envolvendo convolução esférica e translação esférica, podem ser encontrados em [19]. Como os operadores de convolução esférica pertencem à classe de operadores que estamos trabalhando, muitos resultados dessa seção podem ser considerados como generalizações daqueles correspondentes à aproximação por convolução esférica.

O teorema abaixo apresenta duas condições necessárias para que a seqüencia $\left\{T_{n}\right\}_{n \in \mathbb{N}}$ seja uma aproximação da identidade em $X$.

Teorema 3.3.1. Se $\left\{T_{n}\right\}_{n \in \mathbb{N}}$ é uma aproximação da identidade em $X$, então

(i) Existe uma constante $C>0$ tal que $\left\|T_{n}(f)\right\|_{X} \leq C\|f\|_{X}, n \in \mathbb{N}, f \in X$;

(ii) $\lim _{n \rightarrow \infty} a_{k l}(n)=1, k \in \mathbb{N}, l=1,2, \ldots, N(m, k)$.

Demonstração: Suponha que $\lim _{n \rightarrow \infty}\left\|T_{n}(f)-f\right\|_{X}=0, f \in X$. Então, fixada $f \in X$, a seqüência $\left\{\left\|T_{n}(f)\right\|_{X}\right\}_{n \in \mathbb{N}}$ é limitada. Pelo Princípio da Limitação Uniforme ([33],p. 217), existe uma constante $C \geq 0$ tal que

$$
\left\|T_{n}(f)\right\|_{X} \leq C\|f\|_{X}, \quad f \in X, \quad n \in \mathbb{N} .
$$


Note que $C>0$, pois caso contrário $T_{n}(f)=0, f \in X, n \in \mathbb{N}$, o que implicaria

$$
\|f\|_{X}=\lim _{n \rightarrow \infty}\left\|T_{n}(f)-f\right\|_{X}=0, \quad f \in X
$$

uma óbvia contradição. Isso justifica o item $(i)$. Para provar o item $(i i)$, sejam $k \in \mathbb{N}$ e $l \in\{1,2, \ldots, N(m, k)\}$. Utilizando a fórmula (3.2), vemos que

$$
\lim _{n \rightarrow \infty} T_{n} Y_{k l}(y)=\lim _{n \rightarrow \infty} a_{k l}(n) Y_{k l}(y)=Y_{k l}(y) \lim _{n \rightarrow \infty} a_{k l}(n), \quad y \in S^{m}
$$

Se $X=C\left(S^{m}\right)$, nossa hipótese inicial implica que

$$
\lim _{n \rightarrow \infty} T_{n} Y_{k l}(y)=Y_{k l}(y), \quad y \in S^{m}
$$

Como $Y_{k l} \not \equiv 0$, podemos escolher $y_{0} \in S^{m}$ tal que $Y_{k l}\left(y_{0}\right) \neq 0$. Portanto, de (3.4) e (3.5) obtemos

$$
Y_{k l}\left(y_{0}\right)=Y_{k l}\left(y_{0}\right) \lim _{n \rightarrow \infty} a_{k l}(n)
$$

ou seja, $\lim _{n \rightarrow \infty} a_{k l}(n)=1$. Se $X=L^{p}\left(S^{m}\right), 1 \leq p<\infty$, deduzimos analogamente que

$$
\lim _{n \rightarrow \infty} T_{n} Y_{k l}(y)=Y_{k l}(y), \quad y \in S^{m} \backslash \Lambda,
$$

onde $\sigma_{m}(\Lambda)=0$. Logo, usando (3.4), vem que

$$
Y_{k l}(y)=Y_{k l}(y) \lim _{n \rightarrow \infty} a_{k l}(n), \quad y \in S^{m} \backslash \Lambda
$$

Seja $y_{1} \in S^{m} \backslash \Lambda$ tal que $Y_{k l}\left(y_{1}\right) \neq 0$. Note que tal $y_{1}$ existe, pois caso contrário, $Y_{k l} \equiv 0$. Procedendo-se como no caso anterior o resultado segue.

Vejamos agora possíveis recíprocas do teorema anterior.

Teorema 3.3.2. Assuma que $\lim _{n \rightarrow \infty} a_{k l}(n)=1, k \in \mathbb{N}, l=1,2 \ldots, N(m, k)$. Seja $f \in$ $C\left(S^{m}\right)$ satisfazendo a seguinte condição: se $q \in \mathbb{P}\left(S^{m}\right)$, existe $C:=C_{f-q} \geq 0$ tal que $\left\|T_{n}(f-q)\right\|_{\infty} \leq C_{f-q}\|f-q\|_{\infty}, n \in \mathbb{N}$. Então $\lim _{n \rightarrow \infty}\left\|T_{n}(f)-f\right\|_{\infty}=0$.

Demonstração: Seja $\epsilon>0$. O Teorema da Aproximação de Weierstrass ([33], p. 96) garante a existência de um polinômio $q$ tal que $\|f-q\|_{\infty}<\epsilon$. Escrevevendo $q$ na forma

$$
q=\sum_{k=0}^{M} \sum_{l=1}^{N(m, k)} r_{k l} Y_{k l}, \quad r_{k l} \in \mathbb{C}, \quad k=0,1, \ldots, M, \quad l=1,2, \ldots, N(m, k)
$$


e usando (3.3), obtemos

$$
\begin{aligned}
\left|T_{n} q(y)-q(y)\right| & =\left|\sum_{k=0}^{M} \sum_{l=1}^{N(m, k)}\left(a_{k l}(n)-1\right) r_{k l} Y_{k l}(y)\right| \\
& \leq \sum_{k=0}^{M} \sum_{l=1}^{N(m, k)}\left|a_{k l}(n)-1\right|\left|r_{k l}\right|\left|Y_{k l}(y)\right| \\
& \leq \sum_{k=0}^{M} \sum_{l=1}^{N(m, k)}\left|a_{k l}(n)-1\right|\left|r_{k l}\right||| Y_{k l} \|_{\infty} \\
& \leq B_{1} B_{2} \sum_{k=0}^{M} \sum_{l=1}^{N(m, k)}\left|a_{k l}(n)-1\right|, \quad y \in S^{m}, \quad n \geq M
\end{aligned}
$$

onde

$$
B_{1}:=\max \left\{\left|r_{k l}\right|: k=0,1, \ldots, M, l=1,2, \ldots, N(m, k)\right\}
$$

e

$$
B_{2}:=\max \left\{\left\|Y_{k l}\right\|_{\infty}: k=0,1, \ldots, M, l=1,2, \ldots, N(m, k)\right\} .
$$

Conseqüentemente,

$$
\left\|T_{n}(q)-q\right\|_{\infty} \leq B_{1} B_{2} \sum_{k=0}^{M} \sum_{l=1}^{N(m, k)}\left|a_{k l}(n)-1\right|, \quad n \geq M
$$

Pela hipótese sobre as seqüências $\left\{a_{k l}(n)\right\}_{n \in \mathbb{N}}$, segue que

$$
\lim _{n \rightarrow \infty}\left\|T_{n}(q)-q\right\|_{\infty}=0
$$

Logo, existe $N(\epsilon) \in \mathbb{N}$ tal que

$$
\left\|T_{n}(q)-q\right\|_{\infty}<\epsilon, \quad n \geq N(\epsilon)
$$

Assim, temos que

$$
\begin{aligned}
\left\|T_{n}(f)-f\right\|_{\infty} & \leq\left\|T_{n}(f)-T_{n}(q)\right\|_{\infty}+\left\|T_{n} q-q\right\|_{\infty}+\|q-f\|_{\infty} \\
& \leq\left\|T_{n}(f-q)\right\|_{\infty}+2 \epsilon \\
& \leq C_{f-q}\|f-q\|_{\infty}+2 \epsilon \\
& \leq\left(C_{f-q}+2\right) \epsilon, \quad n \geq N(\epsilon),
\end{aligned}
$$

completando a prova do teorema.

Teorema 3.3.3. Assuma que $\lim _{n \rightarrow \infty} a_{k l}(n)=1, k \in \mathbb{N}, l=1,2, \ldots, N(m, k)$. Seja $f \in$ $L^{p}\left(S^{m}\right)$ satisfazendo a seguinte condição: se $q \in \mathbb{P}\left(S^{m}\right)$, existe uma constante $C:=C_{f-q} \geq 0$ tal que $\left\|T_{n}(f-q)\right\|_{p} \leq C_{f-q}\|f-q\|_{p}, n \in \mathbb{N}$. Então $\lim _{n \rightarrow \infty}\left\|T_{n}(f)-f\right\|_{p}=0$. 
Demonstração: Seja $\epsilon>0$. Como $C\left(S^{m}\right)$ é denso em $L^{p}\left(S^{m}\right)$, existe uma função $g \in C\left(S^{m}\right)$ tal que $\|f-g\|_{p}<\epsilon / 2$. Além disso, pelo Teorema da Aproximação de Weierstrass, existe um polinômio $q$ tal que $\|q-g\|_{\infty}<\epsilon / 2$. Logo,

$$
\|f-q\|_{p} \leq\|f-g\|_{p}+\|g-q\|_{p} \leq \frac{\epsilon}{2}+\|g-q\|_{\infty} \leq \frac{\epsilon}{2}+\frac{\epsilon}{2}=\epsilon .
$$

Como na prova do teorema anterior, $\lim _{n \rightarrow \infty}\left\|T_{n}(q)-q\right\|_{\infty}=0$. A desigualdade

$$
\left\|T_{n}(q)-q\right\|_{p} \leq\left\|T_{n}(q)-q\right\|_{\infty}
$$

mostra que $\lim _{n \rightarrow \infty}\left\|T_{n}(q)-q\right\|_{p}=0$. Portanto, existe $N(\epsilon) \in \mathbb{N}$ tal que

$$
\left\|T_{n}(q)-q\right\|_{p}<\epsilon, \quad n \geq N(\epsilon)
$$

Assim,

$$
\begin{aligned}
\left\|T_{n}(f)-f\right\|_{p} & \leq\left\|T_{n}(f)-T_{n}(q)\right\|_{p}+\left\|T_{n}(q)-q\right\|_{p}+\|q-f\|_{p} \\
& \leq\left\|T_{n}(f-q)\right\|_{p}+2 \epsilon \\
& \leq C_{f-q}\|f-q\|_{p}+2 \epsilon \\
& \leq\left(C_{f-q}+2\right) \epsilon, \quad n \geq N(\epsilon),
\end{aligned}
$$

completando a prova do teorema.

O resultado abaixo é conseqüência direta dos três teoremas acima.

Corolário 3.3.4. A seqüência $\left\{T_{n}\right\}_{n \in \mathbb{N}}$ é uma aproximação da identidade em $X$ se, e somente se, valem as seguintes condições:

(i) Para cada $f \in X$, existe uma constante $C_{f} \geq 0$ tal que $\left\|T_{n}(f)\right\|_{X} \leq C_{f}\|f\|_{X}, n \in \mathbb{N}$;

(ii) $\lim _{n \rightarrow \infty} a_{k l}(n)=1, k \in \mathbb{N}, l=1,2, \ldots, N(m, k)$.

A seguir, investigaremos a possibilidade de trocar a hipótese $(i)$ no corolário anterior por outra envolvendo o núcleo $K_{n}$. A suspeita de que tal possibilidade viesse a ocorrer partiu da representação deduzida no Teorema 3.1.1.

Teorema 3.3.5. Se $\lim _{n \rightarrow \infty} a_{k l}(n)=1, k \in \mathbb{N}, l=1,2, \ldots, N(m, k) e$

$$
\sup _{n \in \mathbb{N}}\left(\sup \left\{\left\|K_{n}^{x}\right\|_{1}: x \in S^{m}\right\}\right)<\infty
$$

então $\left\{T_{n}\right\}_{n \in \mathbb{N}}$ é uma aproximação da identidade em $X$.

Demonstração: Se existir uma constante positiva $C$, que não depende de $n$, tal que

$$
\sup \left\{\left\|K_{n}^{x}\right\|_{1}: x \in S^{m}\right\} \leq C, \quad n \in \mathbb{N}
$$


então o Teorema 3.2.1 implica que $\left\|T_{n}\right\|_{X} \leq C, n \in \mathbb{N}$. Daí,

$$
\frac{\left\|T_{n}(f)\right\|_{X}}{\|f\|_{X}} \leq \sup _{f \in X} \frac{\left\|T_{n}(f)\right\|_{X}}{\|f\|_{X}}=\left\|T_{n}\right\|_{X} \leq C, \quad n \in \mathbb{N}, \quad f \in X \backslash\{0\},
$$

e, por conseguinte,

$$
\left\|T_{n}(f)\right\|_{X} \leq C\|f\|_{X}, \quad n \in \mathbb{N}, \quad f \in X
$$

Assim, o teorema segue diretamente do corolário anterior.

A recíproca do teorema acima vale em pelo menos dois casos.

Teorema 3.3.6. Assuma que $X=C\left(S^{m}\right)$ ou $L^{1}\left(S^{m}\right)$. Então $\left\{T_{n}\right\}_{n \in \mathbb{N}}$ é uma aproximação da identidade em $X$ se, e somente se, valem as seguintes condições:

(i) $\sup _{n \in \mathbb{N}}\left(\sup \left\{\left\|K_{n}^{x}\right\|_{1}: x \in S^{m}\right\}\right)<\infty$;

(ii) $\lim _{n \rightarrow \infty} a_{k l}(n)=1, k \in \mathbb{N}, l=1,2, \ldots, N(m, k)$.

Demonstração: Uma implicação é conseqüência do teorema anterior. Para a outra, assuma que $\left\{T_{n}\right\}_{n \in \mathbb{N}}$ é uma aproximação da identidade em $X$. A condição $(i i)$ vale pelo Teorema 3.3.1. Seja $C$ a constante dada pelo Teorema 3.3.1-(i). Então

$$
\left\|T_{n}\right\|_{X}=\sup _{f \in X \backslash\{0\}} \frac{\left\|T_{n}(f)\right\|_{X}}{\|f\|_{X}} \leq C, \quad n \in \mathbb{N} .
$$

Como o Teorema 3.2.2 é aplicável,

$$
\sup \left\{\left\|K_{n}^{x}\right\|_{1}: x \in S^{m}\right\}=\left\|T_{n}\right\|_{X} \leq C, \quad n \in \mathbb{N}
$$

Portanto,

$$
\sup _{n \in \mathbb{N}}\left(\sup \left\{\left\|K_{n}^{x}\right\|_{1}: x \in S^{m}\right\}\right) \leq C
$$

e o teorema segue.

Se $K_{n}$ é um núcleo positivo, isto é, $K_{n}(x, y) \geq 0, x, y \in S^{m}, n \in \mathbb{N}$, então podemos refinar a afirmação feita no teorema acima.

Teorema 3.3.7. Assuma que $X=C\left(S^{m}\right)$ ou $X=L^{1}\left(S^{m}\right)$ e suponha que $K_{n}(x, y) \geq 0$, $x, y \in S^{m}, n \in \mathbb{N}$. Então $\left\{T_{n}\right\}_{n \in \mathbb{N}}$ é uma aproximação da identidade em $X$ se, e somente se, $\lim _{n \rightarrow \infty} a_{k l}(n)=1, k \in \mathbb{N}, l=1,2, \ldots, N(m, k)$.

Demonstração: Uma implicação é conseqüência do Teorema 3.3.5. Para a outra, assumimos a condição nas seqüências $\left\{a_{k l}(n)\right\}$ e procedemos da seguinte forma. A positividade de $K_{n}$ e 
a ortonormalidade dos harmônicos esféricos permite deduzirmos que

$$
\begin{aligned}
\left\|K_{n}^{x}\right\|_{1} & =\frac{1}{\sigma_{m}} \int_{S^{m}} K_{n}(x, y) d \sigma_{m}(y) \\
& =\frac{1}{\sigma_{m}} \int_{S^{m}} \sum_{k=0}^{n} \sum_{l=1}^{N(m, k)} a_{k l}(n) Y_{k l}(x) \overline{Y_{k l}(y)} d \sigma_{m}(y) \\
& =\sum_{k=0}^{n} \sum_{l=1}^{N(m, k)} a_{k l}(n) Y_{k l}(x) \frac{1}{\sigma_{m} Y_{01}(x)} \int_{S^{m}} Y_{01}(y) \overline{Y_{k l}(y)} d \sigma_{m}(y) \\
& =a_{01}(n), \quad x \in S^{m}, \quad n \in \mathbb{N} .
\end{aligned}
$$

Daí,

$$
\sup _{x \in S^{m}}\left\|K_{n}^{x}\right\|_{1}=a_{01}(n), \quad n \in \mathbb{N} .
$$

Como $\lim _{n \rightarrow \infty} a_{01}(n)=1$, segue que

$$
\lim _{n \rightarrow \infty} \sup _{x \in S^{m}}\left\|K_{n}^{x}\right\|_{1}=\lim _{n \rightarrow \infty} a_{01}(n)=1 .
$$

Portanto, existe uma constante positiva $C$ tal que

$$
\sup _{n \in \mathbb{N}}\left(\sup \left\{\left\|K_{n}^{x}\right\|_{1}: x \in S^{m}\right\}\right) \leq C .
$$

Logo, o resultado segue do teorema anterior.

A seguir incluímos conseqüências e reformulações dos resultados acima.

Corolário 3.3.8. Assuma que $X=C\left(S^{m}\right)$ ou $L^{1}\left(S^{m}\right)$ e que $\lim _{n \rightarrow \infty} a_{k l}(n)=1, k \in \mathbb{N}$, $l=1,2, \ldots, N(m, k)$. Então as seguintes afirmações são equivalentes:

(i) $\lim _{n \rightarrow \infty}\left\|T_{n}(f)-f\right\|_{X}=0, f \in X$;

(ii) Existe uma constante $C \geq 0$ tal que $\left\|T_{n}(f)\right\|_{X} \leq C\|f\|_{X}, f \in X, n \in \mathbb{N}$;

(iii) $\sup _{n \in \mathbb{N}}\left(\sup \left\{\left\|K_{n}^{x}\right\|_{1}: x \in S^{m}\right\}<\infty\right.$.

Corolário 3.3.9. A seqüência $\left\{T_{n}\right\}_{n \in \mathbb{N}}$ é uma aproximação da identidade em $C\left(S^{m}\right)$ se, e somente se, é uma aproximação da identidade em $L^{1}\left(S^{m}\right)$.

Observação 3.3.10. Os Teoremas 3.3.5 e 3.3.6 implicam que se $\left\{T_{n}\right\}_{n \in \mathbb{N}}$ é uma aproximação da identidade em $C\left(S^{m}\right)$, então também o é em $L^{p}\left(S^{m}\right), 1 \leq p<\infty$. Dessa forma, fica claro que o contexto mais importante a ser estudado é o caso em que $X=C\left(S^{m}\right)$.

Teorema 3.3.11. Se $X=C\left(S^{m}\right)$ ou $L^{1}\left(S^{m}\right)$ e $\sup _{n \in \mathbb{N}} \sup _{x \in S^{m}}\left\|K_{n}^{x}\right\|_{1}<\infty$, então as seguintes afirmações são equivalentes:

(i) $\lim _{n \rightarrow \infty}\left\|T_{n}(f)-f\right\|_{X}=0, f \in X$;

(ii) $\lim _{n \rightarrow \infty} a_{k l}(n)=1, k \in \mathbb{N}, l=1,2, \ldots, N(m, k)$;

(iii) $\lim _{n \rightarrow \infty}\left\|T_{n}(Y)-Y\right\|_{X}=0, Y \in \cup_{k=0}^{\infty} \mathcal{H}_{k}\left(S^{m}\right)$;

(iv) $\lim _{n \rightarrow \infty}\left\|T_{n}(W)-W\right\|_{X}=0, W \in \oplus_{k=0}^{\infty} \mathcal{H}_{k}\left(S^{m}\right)$. 
Demonstração: Assuma as hipóteses do teorema. Se $(i)$ vale, então (ii) segue diretamente do Teorema 3.3.1. Se (ii) vale, o Teorema 3.3 .5 implica que $\lim _{n \rightarrow \infty}\left\|T_{n}(f)-f\right\|_{X}=0, f \in X$. Em particular, $\lim _{n \rightarrow \infty}\left\|T_{n}(Y)-Y\right\|_{X}=0, Y \in \cup_{k=0}^{\infty} \mathcal{H}_{k}\left(S^{m}\right)$, e (iii) segue. Suponha que (iii) vale e seja $W=\sum_{j=0}^{M} Y_{j}, Y_{j} \in \mathcal{H}_{j}\left(S^{m}\right)$. Da linearidade de $T_{n}$ segue que

$$
\left\|T_{n}(W)-W\right\|_{X} \leq \sum_{j=0}^{M}\left\|T_{n}\left(Y_{j}\right)-Y_{j}\right\|_{X} .
$$

Portanto, tomando-se o limite na desigualdade acima temos que

$$
\lim _{n \rightarrow \infty}\left\|T_{n}(W)-W\right\|_{X}=0, \quad W \in \oplus_{k=0}^{\infty} \mathcal{H}_{k}\left(S^{m}\right)
$$

A prova do Teorema 3.3.5 revela que existe $C \geq 0$ tal que $\left\|T_{n}(f)\right\|_{X} \leq C\|f\|_{X}, f \in X, n \in \mathbb{N}$. Sejam $f \in X$ e $\epsilon>0$. Da fundamentalidade de $\cup_{k=0}^{\infty} \mathcal{H}_{k}\left(S^{m}\right)$ em $X$, existe uma seqüência $\left\{W_{n}\right\}_{n \in \mathbb{N}}$ de elementos de $\oplus_{k=0}^{\infty} \mathcal{H}_{k}\left(S^{m}\right)$ e um índice $n_{0} \in \mathbb{N}$ tal que

$$
\left\|W_{n}-f\right\|_{X}<\frac{\epsilon}{C+2}, \quad n \geq n_{0} .
$$

Se (iv) vale, $\lim _{n \rightarrow \infty}\left\|T_{n}\left(W_{n_{0}}\right)-W_{n_{0}}\right\|_{X}=0$, e podemos escolher um índice $n_{1} \geq n_{0}$ tal que

$$
\left\|T_{n}\left(W_{n_{0}}\right)-W_{n_{0}}\right\|_{X}<\frac{\epsilon}{C+2}, \quad n \geq n_{1} .
$$

Logo,

$$
\begin{aligned}
\left\|T_{n}(f)-f\right\|_{X} & \leq\left\|T_{n}(f)-T_{n}\left(W_{n_{0}}\right)\right\|_{X}+\left\|T_{n}\left(W_{n_{0}}\right)-W_{n_{0}}\right\|_{X}+\left\|W_{n_{0}}-f\right\|_{X} \\
& \leq\left\|T_{n}\left(f-W_{n_{0}}\right)\right\|_{X}+\frac{\epsilon}{C+2}+\frac{\epsilon}{C+2} \\
& \leq C\left\|f-W_{n_{0}}\right\|_{X}+\frac{2 \epsilon}{C+2} \\
& \leq \epsilon, \quad n \geq n_{1} .
\end{aligned}
$$

Portanto, $(i)$ vale.

O teorema abaixo nos fornece um meio de construir aproximações da identidade a partir de outras dadas.

Teorema 3.3.12. Se $\left\{T_{n}\right\}_{n \in \mathbb{N}} e\left\{S_{n}\right\}_{n \in \mathbb{N}}$ são aproximações da identidade em $X$, então $\left\{T_{n} \circ\right.$ $\left.S_{n}\right\}_{n \in \mathbb{N}}$ é uma aproximação da identidade em $X$.

Demonstração: Seja $\left\{T_{n}\right\}_{n \in \mathbb{N}}$ como em (3.1) e $\left\{S_{n}\right\}_{n \in \mathbb{N}}$ dada por

$$
S_{n}(f)=\sum_{i=0}^{n} \sum_{j=1}^{N(m, i)} c_{i j}(n) \hat{f}(i, j) Y_{i j}, \quad f \in X,
$$


$\operatorname{com} c_{i j}(n) \in \mathbb{R}, i \in \mathbb{N}, j=1,2, \ldots, N(m, i)$. Utilizando a representação dada pelo Teorema 3.1.1, vemos que

$$
\begin{aligned}
\left(T_{n}\left(S_{n} f\right)\right)(x) & =\frac{1}{\sigma_{m}} \int_{S^{m}} K_{n}(x, y)\left(S_{n} f\right)(y) d \sigma_{m}(y) \\
& =\frac{1}{\sigma_{m}} \int_{S^{m}} \sum_{k=0}^{n} \sum_{l=1}^{N(m, k)} a_{k l}(n) Y_{k l}(x) \overline{Y_{k l}(y)} \sum_{i=0}^{n} \sum_{j=1}^{N(m, i)} c_{i j}(n) \hat{f}(i, j) Y_{i j}(y) d \sigma_{m}(y) \\
& =\sum_{k=0}^{n} \sum_{l=1}^{N(m, k)} \sum_{i=0}^{n} \sum_{j=1}^{N(m, i)} a_{k l}(n) c_{i j}(n) \hat{f}(i, j) Y_{k l}(x) \frac{1}{\sigma_{m}} \int_{S^{m}} Y_{i j}(y) \overline{Y_{k l}(y)} d \sigma_{m}(y) \\
& =\sum_{k=0}^{n} \sum_{l=1}^{N(m, k)} a_{k l}(n) c_{k l}(n) \hat{f}(k, l) Y_{k l}(x), \quad x \in S^{m}, \quad f \in X .
\end{aligned}
$$

Como $\left\{T_{n}\right\}_{n \in \mathbb{N}}$ e $\left\{S_{n}\right\}_{n \in \mathbb{N}}$ são aproximações da identidade em $X$, o Teorema 3.3.1 garante que

$$
\lim _{n \rightarrow \infty} a_{k l}(n)=\lim _{n \rightarrow \infty} c_{k l}(n)=1, \quad k \in \mathbb{N}, \quad l=1,2, \ldots, N(m, k)
$$

Logo,

$$
\lim _{n \rightarrow \infty} a_{k l}(n) c_{k l}(n)=1, \quad k \in \mathbb{N}, \quad l=1,2, \ldots, N(m, k) .
$$

Pelo mesmo teorema, existem constantes $C_{1}, C_{2}>0$ tais que

$$
\begin{aligned}
& \left\|T_{n}(f)\right\|_{X} \leq C_{1}\|f\|_{X}, \quad n \in \mathbb{N}, \quad f \in X \\
& \left\|S_{n}(f)\right\|_{X} \leq C_{2}\|f\|_{X}, \quad n \in \mathbb{N}, \quad f \in X .
\end{aligned}
$$

Portanto,

$$
\left\|T_{n}\left(S_{n} f\right)\right\|_{X} \leq C_{1}\left\|S_{n}(f)\right\|_{X} \leq C_{1} C_{2}\|f\|_{X}, \quad n \in \mathbb{N}, \quad f \in X .
$$

Pelo Corolário 3.3.4, $\left\{T_{n} \circ S_{n}\right\}_{n \in \mathbb{N}}$ é uma aproximação da identidade em $X$.

Observação 3.3.13. Se $\left\{T_{n}\right\}_{n \in \mathbb{N}}$ é uma aproximação da identidade em $X$, então o mesmo vale para $\left\{T_{n}^{2}\right\}_{n \in \mathbb{N}}$. Portanto, $\left\{T_{n}^{k}\right\}_{n \in \mathbb{N}}, k \in \mathbb{N}$, é aproximação da identidade em $X$.

\subsection{Um exemplo}

Apresentaremos nesta seção um método construtivo para gerar uma seqüência $\left\{K_{n}\right\}$ na qual todos os núcleos são positivos e bi-zonais, e de forma que os operadores associados a esses núcleos dêem origem a uma aproximação da identidade em $X$.

Consideremos o núcleo

$$
F_{n}(\langle x, y\rangle)=\sum_{k=0}^{n} \sum_{l=1}^{N(m, k)} Y_{k l}(x) \overline{Y_{k l}(y)}, \quad x, y \in S^{m} .
$$


Pelo Teorema da Adição podemos escrever

$$
F_{n}(\langle x, y\rangle)=\sum_{k=0}^{n} N(m, k) P_{k}^{m}(\langle x, y\rangle) .
$$

Calculando o quadrado de $F_{n}$ e usando a fórmula de linearização de Dougall dada no Capítulo 1 , obtemos

$$
\begin{aligned}
\left(F_{n}(\langle x, y\rangle)\right)^{2} & =\sum_{k=0}^{n} \sum_{i=0}^{n} N(m, k) N(m, i) P_{k}^{m}(\langle x, y\rangle) P_{i}^{m}(\langle x, y\rangle) \\
& =\sum_{k=0}^{n} \sum_{i=0}^{n} \sum_{j=|k-i|}^{k+i} N(m, k) N(m, i) d(k, i, j) P_{j}^{m}(\langle x, y\rangle) .
\end{aligned}
$$

Fazendo uma mudança na ordem das somas segue que

$$
\begin{aligned}
\left(F_{n}(\langle x, y\rangle)\right)^{2} & =\sum_{k=0}^{n} \sum_{j=0}^{k+n} \sum_{i=|k-j|}^{k+j \wedge n} N(m, k) N(m, i) d(k, i, j) P_{j}^{m}(\langle x, y\rangle) \\
& =\sum_{j=0}^{2 n} \sum_{k=0}^{n} \sum_{i=|k-j|}^{k+j \wedge n} N(m, k) N(m, i) d(k, i, j) P_{j}^{m}(\langle x, y\rangle),
\end{aligned}
$$

onde $k+j \wedge n=\min \{k+j, n\}$. Usando novamente o Teorema da Adição temos que

$$
\begin{aligned}
\left(F_{n}(\langle x, y\rangle)\right)^{2} & =\sum_{j=0}^{2 n} \sum_{l=1}^{N(m, j)} \sum_{k=0}^{n} \sum_{i=|k-j|}^{k+j \wedge n} N(m, k) N(m, i) \frac{d(k, i, j)}{N(m, j)} Y_{j l}(x) \overline{Y_{j l}(y)} \\
& =\sum_{j=0}^{2 n} \sum_{l=1}^{N(m, j)} b_{j}(2 n) Y_{j l}(x) \overline{Y_{j l}(y)}
\end{aligned}
$$

onde

$$
b_{j}(2 n):=\sum_{k=0}^{n} \sum_{i=|k-j|}^{k+j \wedge n} N(m, k) N(m, i) \frac{d(k, i, j)}{N(m, j)} .
$$

Em particular, a Proposição 1.3 .7 garante que

$$
b_{0}(2 n)=\sum_{k=0}^{n} N(m, k)^{2} d(k, k, 0)=\sum_{k=0}^{n} N(m, k) .
$$

Como $b_{0}(2 n)>0, n \in \mathbb{N}$, podemos definir o núcleo

$$
K_{2 n}(\langle x, y\rangle):=\frac{\left(F_{n}(\langle x, y\rangle)\right)^{2}}{b_{0}(2 n)}=\sum_{j=0}^{2 n} \sum_{l=1}^{N(m, j)} a_{j}(2 n) Y_{j l}(x) \overline{Y_{j l}(y)}
$$

onde

$$
a_{j}(2 n):=\frac{b_{j}(2 n)}{b_{0}(2 n)}
$$


Notemos que $K_{2 n}$ assim construído é um núcleo positivo e bizonal. Associado a esse núcleo, definimos o operador $T_{2 n}: X \rightarrow X$ por

$$
\begin{aligned}
T_{2 n}(f)(x) & =\frac{1}{\sigma_{m}} \int_{S^{m}} K_{2 n}(\langle x, y\rangle) f(y) d \sigma_{m}(y) \\
& =\sum_{j=0}^{2 n} \sum_{l=1}^{N(m, j)} a_{j}(2 n) \hat{f}(j, l) Y_{j l}(x), \quad x \in S^{m} .
\end{aligned}
$$

Observemos que como $K_{2 n}(\langle x, y\rangle) \geq 0, x, y \in S^{m}$, segue do Teorema 3.3.7 que $\left\{T_{2 n}\right\}_{n \in \mathbb{N}}$ é uma aproximação da identidade em $X$ se $\lim _{n \rightarrow \infty} a_{j}(2 n)=1, j \in \mathbb{N}$. O lema abaixo fornece uma condição necessária e suficiente para que isso ocorra.

Lema 3.4.1. Com as notações acima, $\lim _{n \rightarrow \infty} a_{j}(2 n)=1, j \in \mathbb{N}$, se, e somente se

$$
\lim _{n \rightarrow \infty} \frac{1}{d_{n}} \sum_{k=n+1}^{n} \sum_{i=n+1}^{k+j} N(m, k) d(j, k, i)=0, \quad j \in \mathbb{N} .
$$

Demonstração: A definição de $a_{j}(2 n)$ e a Equação (1.14) implicam que

$$
\begin{aligned}
a_{j}(2 n) & =\frac{1}{d_{n}} \sum_{k=0}^{n} \sum_{i=|k-j|}^{k+j \wedge n} N(m, k) N(m, i) d(k, i, j) N(m, j)^{-1} \\
& =\frac{1}{d_{n}} \sum_{k=0}^{n} \sum_{i=|k-j|}^{k+j \wedge n} N(m, k) d(j, k, i) .
\end{aligned}
$$

Usando o Teorema 1.3.5 podemos escrever, para $n \geq j$,

$$
\begin{aligned}
a_{j}(2 n) & =\frac{1}{d_{n}} \sum_{k=0}^{n-j} \sum_{i=|k-j|}^{k+j} N(m, k) d(j, k, i)+\frac{1}{d_{n}} \sum_{k=n+1-j}^{n} \sum_{i=|k-j|}^{n} N(m, k) d(j, k, i) \\
& =\frac{1}{d_{n}} \sum_{k=0}^{n-j} N(m, k)+\frac{1}{d_{n}} \sum_{k=n+1-j}^{n} \sum_{i=|k-j|}^{n} N(m, k) d(j, k, i) .
\end{aligned}
$$

Mas, usando novamente o Teorema 1.3.5, a última soma da equação acima pode ser reescrita da seguinte forma

$$
\begin{aligned}
\sum_{k=n+1}^{n} \sum_{i=|k-j|}^{n} N(m, k) d(j, k, i) & =\sum_{k=n+1-j}^{n} N(m, k)\left(\sum_{i=|k-j|}^{k+j} d(j, k, i)-\sum_{i=n+1}^{k+j} d(j, k, i)\right) \\
& =\sum_{k=n+1-j}^{n} N(m, k)-\sum_{k=n+1-j}^{n} \sum_{i=n+1}^{k+j} N(m, k) d(j, k, i)
\end{aligned}
$$

Portanto,

$$
\begin{aligned}
a_{j}(2 n) & =\frac{1}{d_{n}} \sum_{k=0}^{n} N(m, k)-\frac{1}{d_{n}} \sum_{k=n+1-j}^{n} \sum_{i=n+1}^{k+j} N(m, k) d(j, k, i) \\
& =1-\frac{1}{d_{n}} \sum_{k=n+1-j}^{n} \sum_{i=n+1}^{k+j} N(m, k) d(j, k, i) .
\end{aligned}
$$


Logo, $\lim _{n \rightarrow \infty} a_{j}(2 n)=1, j \in \mathbb{N}$, se, e somente se

$$
\lim _{n \rightarrow \infty} \frac{1}{d_{n}} \sum_{k=n+1}^{n} \sum_{-j=n+1}^{k+j} N(m, k) d(j, k, i)=0, \quad j \in \mathbb{N},
$$

e o lema está provado.

O próximo teorema revela que $\left\{T_{2 n}\right\}_{n \in \mathbb{N}}$ é uma aproximação da identidade em $X$.

Teorema 3.4.2. Nas notações acima, $\lim _{n \rightarrow \infty} a_{j}(2 n)=1, j \in \mathbb{N}$ e, portanto, a seqüência de operadores $\left\{T_{2 n}\right\}_{n \in \mathbb{N}}$ definida em (3.7) é uma aproximação da identidade em $X$.

Demonstração: Do lema anterior segue que é suficiente provar que

$$
\lim _{n \rightarrow \infty} \frac{1}{d_{n}} \sum_{k=n+1}^{n} \sum_{-j=n+1}^{k+j} N(m, k) d(j, k, i)=0, \quad j \in \mathbb{N} .
$$

Usando o Teorema 1.3.5 temos que

$$
\begin{aligned}
0 \leq \frac{1}{d_{n}} \sum_{k=n+1}^{n} \sum_{i=n+1}^{k+j} N(m, k) d(j, k, i) & \leq \frac{1}{d_{n}} \sum_{k=n+1-j}^{n} N(m, k) \sum_{i=|k-j|}^{k+j} d(j, k, i) \\
& =\frac{1}{d_{n}} \sum_{k=n+1-j}^{n} N(m, k)
\end{aligned}
$$

A soma do último termo da desigualdade acima tem $j$ termos para todo $n \in \mathbb{N}$. Logo, para terminar a prova, basta calcular

$$
\lim _{n \rightarrow \infty} \frac{N(m, k)}{d_{n}}, \quad k=n+1-j, \ldots, n
$$

Como $k \leq n$, usando a Proposição 1.1.7, segue que

$$
\lim _{n \rightarrow \infty} \frac{N(m, k)}{d_{n}} \leq \lim _{n \rightarrow \infty} \frac{N(m, n)}{d_{n}}=0 .
$$

Portanto,

$$
\lim _{n \rightarrow \infty} \frac{N(m, k)}{d_{n}}=0, \quad k=n+1-j, \ldots, n
$$

Logo,

$$
\lim _{n \rightarrow \infty} \frac{1}{d_{n}} \sum_{k=n+1}^{n} \sum_{-j=n+1}^{k+j} N(m, k) d(j, k, i)=0, \quad j \in \mathbb{N} .
$$

Isso completa a prova do teorema. 


\subsection{Aproximação da identidade: o caso pontual}

Nesta seção, consideraremos somente o caso em que $X=C\left(S^{m}\right)$. Investigaremos aproximações similares àquelas consideradas na seção anterior, tentando na medida do possível estabelecer versões pontuais dos resultados daquela seção.

Para motivar a definição principal desta seção seja $\left\{T_{n}\right\}$ como em (3.1) e assuma que $\left\{T_{n}\right\}_{n \in \mathbb{N}}$ é uma aproximação da identidade em $C\left(S^{m}\right)$. Como $\lim _{n \rightarrow \infty}\left\|T_{n}(f)-f\right\|_{\infty}=0$, $f \in C\left(S^{m}\right)$, é óbvio que $\lim _{n \rightarrow \infty} T_{n} f(y)=f(y), y \in S^{m}, f \in C\left(S^{m}\right)$. Logo,

$$
\begin{aligned}
f(y) & =\lim _{n \rightarrow \infty} \sum_{k=0}^{n} \sum_{l=1}^{N(m, k)} a_{k l}(n) \hat{f}(k, l) Y_{k l}(y) \\
& =\lim _{n \rightarrow \infty} \frac{1}{\sigma_{m}} \int_{S^{m}} \sum_{k=0}^{n} \sum_{l=1}^{N(m, k)} a_{k l}(n) Y_{k l}(y) \overline{Y_{k l}(x)} f(x) d \sigma_{m}(x) \\
& =\lim _{n \rightarrow \infty} \frac{1}{\sigma_{m}} \int_{S^{m}} T_{n}^{y}(x) f(x) d \sigma_{m}(x), \quad y \in S^{m}, \quad f \in C\left(S^{m}\right) .
\end{aligned}
$$

onde $c_{k l}^{y}(n):=a_{k l}(n) Y_{k l}(y) \mathrm{e}$

$$
T_{n}^{y}(x):=\sum_{k=0}^{n} \sum_{l=1}^{N(m, k)} c_{k l}^{y}(n) \overline{Y_{k l}(x)}, \quad x \in S^{m} .
$$

Dessa forma, introduzimos a seguinte definição.

Definição 3.5.1. Fixado $y \in S^{m}$, dizemos que uma seqüência $\left\{T_{n}^{y}\right\}_{n \in \mathbb{N}}$ dada por

$$
T_{n}^{y}(x):=\sum_{k=0}^{n} \sum_{l=1}^{N(m, k)} b_{k l}^{y}(n) \overline{Y_{k l}(x)}, \quad x \in S^{m},
$$

onde $b_{k l}^{y}(n) \in \mathbb{C}, k=0,1, \ldots, n, l=1,2, \ldots, N(m, k)$, é uma aproximação da identidade em y quando

$$
\lim _{n \rightarrow \infty} \frac{1}{\sigma_{m}} \int_{S^{m}} T_{n}^{y}(x) f(x) d \sigma_{m}(x)=f(y), \quad f \in C\left(S^{m}\right) .
$$

Obviamente, temos o seguinte resultado.

Teorema 3.5.2. Se $y \in S^{m}$ e $\left\{T_{n}\right\}_{n \in \mathbb{N}}$ como em (3.1) é uma aproximação da identidade em $C\left(S^{m}\right)$, então a seqüência $\left\{T_{n}^{y}\right\}_{n \in \mathbb{N}}$ onde

$$
b_{k l}^{y}(n)=a_{k l}(n) Y_{k l}(y), \quad k \in \mathbb{N}, \quad l=1,2, \ldots, N(m, k)
$$

é uma aproximação da identidade em $y \in S^{m}$.

No que segue, procuraremos condições para que uma seqüência $\left\{T_{n}^{y}\right\}_{n \in \mathbb{N}}$ seja uma aproximação da identidade em $y$. Começamos calculando a norma do operador definido em (3.8). 
Teorema 3.5.3. Seja $L_{n}^{y}$ o funcional linear sobre $C\left(S^{m}\right)$ dado pela expressão

$$
L_{n}^{y}(f)=\frac{1}{\sigma_{m}} \int_{S^{m}} T_{n}^{y}(x) f(x) d \sigma_{m}(x), \quad f \in C\left(S^{m}\right),
$$

com $T_{n}^{y}$ dado pela Fórmula (3.8). Então $L_{n}^{y}$ é contínuo e $\left\|L_{n}^{y}\right\|_{C\left(S^{m}\right)}=\left\|T_{n}^{y}\right\|_{1}$.

Demonstração: Temos que

$$
\begin{aligned}
\left|L_{n}^{y}(f)\right| & =\left|\frac{1}{\sigma_{m}} \int_{S^{m}} T_{n}^{y}(x) f(x) d \sigma_{m}(x)\right| \\
& \leq \frac{1}{\sigma_{m}} \int_{S^{m}}\left|T_{n}^{y}(x) \| f(x)\right| d \sigma_{m}(x) \\
& \leq\|f\|_{\infty}\left\|T_{n}^{y}\right\|_{1}, \quad f \in C\left(S^{m}\right)
\end{aligned}
$$

Logo,

$$
\left\|L_{n}^{y}\right\|_{C\left(S^{m}\right)}=\sup \left\{\left|L_{n}^{y}(f)\right|:\|f\|_{\infty} \leq 1\right\} \leq\left\|T_{n}^{y}\right\|_{1} .
$$

Para obter a outra desigualdade, consideremos o conjunto fechado

$$
F=\left\{x \in S^{m}: T_{n}^{y}(x)=0\right\}
$$

Dado $\epsilon>0$, existe um conjunto aberto $O_{\epsilon} \supset F$ tal que

$$
\frac{1}{\sigma_{m}} \int_{O_{\epsilon}}\left|T_{n}^{y}(x)\right| d \sigma_{m}(x)<\epsilon
$$

Como $S^{m}$ é um espaço normal, segue do Lema de Urysohn ([25]) que existe uma função contínua $g_{\epsilon}: S^{m} \rightarrow[0,1]$ tal que $g_{\epsilon}(x)=0, x \in F$ e $g_{\epsilon}(x)=1, x \in S^{m} \backslash O_{\epsilon}$. Seja $h_{\epsilon}$ a função definida por $h_{\epsilon}(x)=g_{\epsilon}(x) \operatorname{sign} \overline{T_{n}^{y}(x)}$, onde $\operatorname{sign} z=z /|z|, z \neq 0$ e $\operatorname{sign} 0=0$. Note que $h_{\epsilon} \in C\left(S^{m}\right)$ e $\left\|h_{\epsilon}\right\|_{\infty} \leq 1$. Além disso,

$$
\begin{aligned}
L_{n}^{y}\left(h_{\epsilon}\right) & =\frac{1}{\sigma_{m}} \int_{S^{m}} T_{n}^{y}(x) h_{\epsilon}(x) d \sigma_{m}(x) \\
& =\frac{1}{\sigma_{m}} \int_{S^{m}} T_{n}^{y}(x) \operatorname{sign} \overline{T_{n}^{y}(x)} g_{\epsilon}(x) d \sigma_{m}(x) \\
& =\frac{1}{\sigma_{m}} \int_{S^{m} \backslash F}\left|T_{n}^{y}(x)\right| g_{\epsilon}(x) d \sigma_{m}(x) \\
& \geq \frac{1}{\sigma_{m}} \int_{S^{m} \backslash O_{\epsilon}}\left|T_{n}^{y}(x)\right| d \sigma_{m}(x) \\
& \geq\left\|T_{n}^{y}\right\|_{1}-\epsilon .
\end{aligned}
$$

Logo, $\left\|L_{n}^{y}\right\|_{C\left(S^{m}\right)} \geq\left\|T_{n}^{y}\right\|_{1}-\epsilon$. Isso completa a prova do teorema.

Substituindo-se a expressão que define $T_{n}^{y}$ na integral que define $L_{n}^{y}$ e efetuando-se as contas, chegamos à seguinte formulação alternativa:

$$
L_{n}^{y}(f)=\sum_{k=0}^{n} \sum_{l=1}^{N(m, k)} b_{k l}^{y}(n) \hat{f}(k, l), \quad f \in C\left(S^{m}\right) .
$$


A existência de aproximações da identidade pontuais segue dos resultados das seções anteriores e do Teorema 3.5.2. O teorema abaixo fornece uma prova independente desse mesmo resultado.

Teorema 3.5.4. Fixado $y \in S^{m}$, existe uma aproximação da identidade em y.

Demonstração: Seja $y \in S^{m}$. Considere a seguinte família de conjuntos abertos de $\mathbb{R}^{m+1}$ :

$$
O_{n}^{y}=\left\{x \in S^{m}:\langle y-x, y-x\rangle<1 / n^{2}\right\}, \quad n \in \mathbb{N} .
$$

Como $O_{n}^{y}$ é aberto em $\mathbb{R}^{m+1}$, o Lema de Urysohn ([25]) implica que podemos encontrar uma seqüência de funções $\left\{g_{n}^{y}\right\} \subset C\left(S^{m}\right)$ tais que $g_{n}^{y}(x) \geq 0, x \in S^{m}, g_{n}^{y}(x)=0, x \in S^{m} \backslash O_{n}^{y} \mathrm{e}$

$$
\frac{1}{\sigma_{m}} \int_{S^{m}} g_{n}^{y} d \sigma_{m}=1
$$

Por outro lado, o Teorema da Aproximação de Weierstrass garante a existência de uma família de polinômios $\left\{q_{n}^{y}\right\}_{n \in \mathbb{N}}$ tal que

$$
\left\|g_{n}^{y}-q_{n}^{y}\right\|_{\infty}<\frac{1}{n}, \quad n \in \mathbb{N}
$$

Seja $f \in C\left(S^{m}\right)$ e fixe $\epsilon>0$. Da continuidade de $f$ segue que existe $n_{0}=n_{0}(\epsilon) \in \mathbb{N}$ tal que

$$
|f(y)-f(x)|<\epsilon, \quad x \in O_{n_{0}}^{y} .
$$

Além disso, podemos escolher $n_{1} \geq n_{0}$ tal que

$$
|f(y)-f(x)|<\epsilon, \quad x \in O_{n_{1}}^{y}
$$

e $\|f\|_{\infty}<\epsilon n_{1}$. Como

$$
\begin{aligned}
\left|f(y)-\frac{1}{\sigma_{m}} \int_{S^{m}} q_{n}^{y}(x) f(x) d \sigma_{m}(x)\right| & \leq\left|\frac{1}{\sigma_{m}} \int_{S^{m}}(f(y)-f(x)) g_{n}^{y}(x) d \sigma_{m}(x)\right| \\
& +\left|\frac{1}{\sigma_{m}} \int_{S^{m}}\left(g_{n}^{y}(x)-q_{n}^{y}(x)\right) f(x) d \sigma_{m}(x)\right| \\
\leq & \frac{1}{\sigma_{m}} \int_{S^{m}}|f(y)-f(x)| g_{n}^{y}(x) d \sigma_{m}(x) \\
& +\frac{1}{\sigma_{m}} \int_{S^{m}}\left|g_{n}^{y}(x)-q_{n}^{y}(x)\right||f(x)| d \sigma_{m}(x),
\end{aligned}
$$

então

$$
\begin{aligned}
\left|f(y)-\frac{1}{\sigma_{m}} \int_{S^{m}} q_{n}^{y}(x) f(x) d \sigma_{m}(x)\right| & \leq \frac{1}{\sigma_{m}} \int_{O_{n}^{y}}|f(y)-f(x)| g_{n}^{y}(x) d \sigma_{m}(x) \\
& +\left\|g_{n}^{y}-q_{n}^{y}\right\|_{\infty}\|f\|_{\infty} \\
& \leq \epsilon \frac{1}{\sigma_{m}} \int_{O_{n}^{y}} g_{n}^{y}(x) d \sigma_{m}(x)+\frac{\|f\|_{\infty}}{n} \\
& \leq 2 \epsilon, \quad n \geq n_{1} .
\end{aligned}
$$


Como $q_{n}^{y}$ pode obviamente ser escrito na forma

$$
q_{n}^{y}(x)=\sum_{k=0}^{M_{n}} \sum_{l=1}^{N(m, k)} b_{k l}^{y}(n) \overline{Y_{k l}(x)}
$$

onde $M_{n}$ é o grau de $q_{n}^{y}$, o resultado segue.

O teorema abaixo fornece uma caracterização completa de uma aproximação da identidade em $y \in S^{m}$.

Teorema 3.5.5. Seja y $\in S^{m}$. Então $\left\{T_{n}^{y}\right\}_{n \in \mathbb{N}}$ é uma aproximação da identidade em y se, e somente se, as seguintes condições estão satisfeitas:

(i) Existe uma constante positiva $C$ tal que $\left\|T_{n}^{y}\right\|_{1} \leq C, n \in \mathbb{N}$;

(ii) $\lim _{n \rightarrow \infty} b_{k l}^{y}(n)=Y_{k l}(y), k \in \mathbb{N}, l=1,2, \ldots, N(m, k)$.

Demonstração: Suponhamos inicialmente que $\left\{T_{n}^{y}\right\}_{n \in \mathbb{N}}$ é aproximação da identidade em $y$. Então

$$
\lim _{n \rightarrow \infty} L_{n}^{y}(f)=\lim _{n \rightarrow \infty} \frac{1}{\sigma_{m}} \int_{S^{m}} T_{n}^{y}(x) f(x) d \sigma_{m}(x)=f(y), \quad f \in C\left(S^{m}\right) .
$$

Logo, cada seqüência $\left\{L_{n}^{y}(f)\right\}_{n \in \mathbb{N}}$ é limitada e, conseqüentemente, para cada $f \in C\left(S^{m}\right)$ existe uma constante $C_{f} \geq 0$ tal que

$$
\sup \left\{\left|L_{n}^{y}(f)\right|: n \in \mathbb{N}\right\}<C_{f} .
$$

Do Princípio da Limitação Uniforme e do Teorema 3.5.3, segue que

$$
\left\|T_{n}^{y}\right\|_{1}=\left\|L_{n}^{y}\right\|_{C\left(S^{m}\right)}<C, \quad n \in \mathbb{N}
$$

para algum $C>0$. Como,

$$
\begin{aligned}
L_{n}^{y}\left(Y_{\mu \nu}\right) & =\frac{1}{\sigma_{m}} \int_{S^{m}} T_{n}^{y}(x) Y_{\mu \nu}(x) d \sigma_{m}(x) \\
& =\sum_{k=0}^{n} \sum_{l=1}^{N(m, k)} b_{k l}^{y}(n) \frac{1}{\sigma_{m}} \int_{S^{m}} Y_{\mu \nu}(x) \overline{Y_{k l}(x)} d \sigma_{m}(x) \\
& =b_{\mu \nu}^{y}(n), \quad n \geq \mu
\end{aligned}
$$

então

$$
\lim _{n \rightarrow \infty} b_{\mu \nu}^{y}(n)=\lim _{n \rightarrow \infty} L_{n}^{y}\left(Y_{\mu \nu}\right)=Y_{\mu \nu}, \quad \mu \in \mathbb{N}, \quad \nu=1,2, \ldots, N(m, \mu) .
$$

Reciprocamente, suponha que $(i)$ e $(i i)$ valem. Seja $f \in C\left(S^{m}\right)$ e fixe $\epsilon>0$. Pelo Teorema da Aproximação de Weierstrass, existe um polinômio $q$ em $m+1$ variáveis tal que

$$
\|q-f\|_{\infty}<\epsilon .
$$

Escrevendo

$$
q=\sum_{\mu=0}^{M} \sum_{\nu=1}^{N(m, \mu)} r_{\mu \nu} Y_{\mu \nu}
$$


onde $M$ é o grau de $q$, então

$$
\begin{aligned}
L_{n}^{y}(q) & =\sum_{k=0}^{n} \sum_{l=1}^{N(m, k)} b_{k l}^{y}(n) \hat{q}(k, l) \\
& =\frac{1}{\sigma_{m}} \sum_{k=0}^{n} \sum_{l=1}^{N(m, k)} b_{k l}^{y}(n) \int_{S^{m}} q(x) \overline{Y_{k l}(x)} d \sigma_{m}(x) \\
& =\sum_{k=0}^{n} \sum_{l=1}^{N(m, k)} b_{k l}^{y}(n) \sum_{\mu=0}^{M} \sum_{\nu=1}^{N(m, \mu)} r_{\mu \nu} \frac{1}{\sigma_{m}} \int_{S^{m}} Y_{\mu \nu}(x) \overline{Y_{k l}(x)} d \sigma_{m}(x) \\
& =\sum_{k=0}^{M} \sum_{l=1}^{N(m, k)} b_{k l}^{y}(n) r_{k l}, \quad n \geq M .
\end{aligned}
$$

Usando a condição (ii), vem que

$$
\lim _{n \rightarrow \infty} L_{n}^{y}(q)=\lim _{n \rightarrow \infty} \sum_{k=0}^{M} \sum_{l=1}^{N(m, k)} b_{k l}^{y}(n) r_{k l}=\sum_{k=0}^{M} \sum_{l=1}^{N(m, k)} r_{k l} Y_{k l}(y)=q(y) .
$$

Logo, se $N(\epsilon) \in \mathbb{N}$ é tal que

$$
\left|L_{n}^{y}(q)-q(y)\right|<\epsilon, \quad n \geq N(\epsilon)
$$

podemos usar a condição $(i)$ para obter

$$
\begin{aligned}
\left|L_{n}^{y}(f)-f(y)\right| & \leq\left|L_{n}^{y}(f)-L_{n}^{y}(q)\right|+\left|L_{n}^{y}(q)-q(y)\right|+|q(y)-f(y)| \\
& \leq\left|L_{n}^{y}(f-q)\right|+\epsilon+\|q-f\|_{\infty} \\
& \leq\left\|L_{n}^{y}\right\|_{C\left(S^{m}\right)}\|(f-q)\|_{\infty}+2 \epsilon \\
& =\left\|T_{n}^{y}\right\|_{1}\|(f-q)\|_{\infty}+2 \epsilon \\
& \leq(C+2) \epsilon, \quad n \geq N(\epsilon) .
\end{aligned}
$$

Isto completa a prova do teorema.

Podemos estabelecer e provar um resultado semelhante ao Teorema 3.3.7, se assumirmos que $T_{n}^{y}(x) \geq 0, x \in S^{m}, n \in \mathbb{N}$.

Teorema 3.5.6. Seja $y \in S^{m}$ e suponha que $T_{n}^{y}(x) \geq 0, x \in S^{m}, n \in \mathbb{N}$. Então $\left\{T_{n}^{y}\right\}_{n \in \mathbb{N}}$ é aproximação da identidade em y se, e somente se, $\lim _{n \rightarrow \infty} b_{k l}^{y}(n)=Y_{k l}(y), k \in \mathbb{N}, l=$ $1,2, \ldots, N(m, k)$.

Demonstração: Da positividade de $T_{n}^{y}$ e da ortogonalidade dos harmônicos esféricos segue 
que

$$
\begin{aligned}
\left\|T_{n}^{y}\right\|_{1} & =\frac{1}{\sigma_{m}} \int_{S^{m}} T_{n}^{y}(x) d \sigma_{m}(x) \\
& =\frac{1}{\sigma_{m}} \int_{S^{m}} \sum_{k=0}^{n} \sum_{l=1}^{N(m, k)} b_{k l}^{y}(n) \overline{Y_{k l}(x)} d \sigma_{m}(x) \\
& =\sum_{k=0}^{n} \sum_{l=1}^{N(m, k)} b_{k l}^{y}(n) \frac{1}{\sigma_{m} Y_{01}(y)} \int_{S^{m}} \overline{Y_{k l}(x)} Y_{01}(x) d \sigma_{m}(x) \\
& =\frac{b_{01}^{y}(n)}{Y_{01}(y)} .
\end{aligned}
$$

Da hipótese segue que

$$
\lim _{n \rightarrow \infty}\left\|T_{n}^{y}\right\|_{1}=\lim _{n \rightarrow \infty} \frac{b_{01}^{y}(n)}{Y_{01}(y)}=1,
$$

Logo, existe uma constante positiva $C$ tal que $\left\|T_{n}^{y}\right\|_{1} \leq C, n \in \mathbb{N}$. Portanto, o resultado segue do Teorema 3.5.5.

Finalizamos essa seção, apresentando um resultado de aproximação global envolvendo o operador $L_{n}: C\left(S^{m}\right) \rightarrow C\left(S^{m}\right)$ definido por

$$
L_{n}(f)(y):=\frac{1}{\sigma_{m}} \int_{S^{m}} T_{n}^{y}(x) f(x) d \sigma_{m}(x), \quad y \in S^{m} .
$$

A prova de tal resultado é análoga à do Teorema 3.5.5. Entretanto, como essa prova apresenta alguns detalhes diferentes, faremos todos os passos novamente.

Teorema 3.5.7. Seja $L_{n}$ o operador definido acima e assuma que $b_{k l}^{y}(n)$ é contínuo em $y \in S^{m}, k \in \mathbb{N}, l=1,2, \ldots, N(m, k)$. Então $\lim _{n \rightarrow \infty}\left\|L_{n}(f)-f\right\|_{\infty}=0, f \in C\left(S^{m}\right)$, se, e somente se, as seguintes condições estão satisfeitas:

(i) Existe uma constante positiva $C$ tal que $\left\|L_{n}(f)\right\|_{\infty} \leq C\|f\|_{\infty}, f \in C\left(S^{m}\right), n \in \mathbb{N}$;

(ii) $\lim _{n \rightarrow \infty} b_{k l}^{y}(n)=Y_{k l}(y), k \in \mathbb{N}, l=1,2, \ldots, N(m, k)$, uniformemente em $S^{m}$.

Demonstração: Suponhamos que $\lim _{n \rightarrow \infty}\left\|L_{n}(f)-f\right\|_{\infty}=0, f \in C\left(S^{m}\right)$. Então

$$
\lim _{n \rightarrow \infty} L_{n}(f)=f, \quad f \in C\left(S^{m}\right),
$$

o que implica que para cada $f \in C\left(S^{m}\right)$ existe uma constante $C_{f} \geq 0$ tal que

$$
\sup \left\{\left\|L_{n}(f)\right\|_{\infty}: n \in \mathbb{N}\right\}<C_{f} .
$$

Do Princípio da Limitação Uniforme segue que

$$
\left\|L_{n}(f)\right\|_{\infty} \leq C\|f\|_{\infty}, \quad f \in C\left(S^{m}\right), \quad n \in \mathbb{N},
$$

para algum $C>0$. Para provar $(i)$, seja $f \in C\left(S^{m}\right)$. Como $\lim _{n \rightarrow \infty}\left\|L_{n}(f)-f\right\|_{\infty}=0$, dado $\epsilon>0$, existe $N=N(\epsilon, f) \in \mathbb{N}$ tal que

$$
\left\|L_{n}(f)-f\right\|_{\infty}<\epsilon, \quad n \geq N
$$


Isso implica que

$$
\left|L_{n}(f)(y)-f(y)\right|<\epsilon, \quad n \geq N, \quad y \in S^{m} .
$$

Logo, $\lim _{n \rightarrow \infty} L_{n}(f)(y)=f(y)$ uniformemente em $S^{m}$. Em particular,

$$
\lim _{n \rightarrow \infty} L_{n}\left(Y_{k l}\right)(y)=Y_{k l}(y), \quad k \in \mathbb{N}, \quad l=1,2, \ldots, N(m, k),
$$

uniformemente em $S^{m}$. Mas, para $n \geq k$,

$$
L_{n}\left(Y_{k l}\right)(y)=b_{k l}^{y}(n), \quad k \in \mathbb{N}, \quad l=1,2, \ldots, N(m, k) .
$$

Portanto,

$$
\lim _{n \rightarrow \infty} b_{k l}^{y}(n)=\lim _{n \rightarrow \infty} L_{n}\left(Y_{k l}\right)(y)=Y_{k l}(y), \quad k \in \mathbb{N}, \quad l=1,2, \ldots, N(m, k),
$$

uniformemente em $S^{m}$. Reciprocamente, suponha que $(i)$ e $(i i)$ valem. Seja $f \in C\left(S^{m}\right)$ e fixe $\epsilon>0$. Pelo Teorema da Aproximação de Weierstrass, existe um polinômio $q$ em $m+1$ variáveis tal que $\|q-f\|_{\infty}<\epsilon$. Escrevendo

$$
q=\sum_{\mu=0}^{M} \sum_{\nu=1}^{N(m, \mu)} r_{\mu \nu} Y_{\mu \nu}
$$

onde $M$ é o grau de $q$, segue que

$$
L_{n}(q)(y)=\sum_{k=0}^{M} \sum_{l=1}^{N(m, k)} b_{k l}^{y}(n) r_{k l}, \quad n \geq M .
$$

Usando a condição $(i i)$, temos que

$$
\lim _{n \rightarrow \infty} L_{n}(q)(y)=\lim _{n \rightarrow \infty} \sum_{k=0}^{M} \sum_{l=1}^{N(m, k)} b_{k l}^{y}(n) r_{k l}=\sum_{k=0}^{M} \sum_{l=1}^{N(m, k)} r_{k l} Y_{k l}(y)=q(y),
$$

uniformemente em $S^{m}$. Logo, existe $N(\epsilon) \in \mathbb{N}$ tal que

$$
\left|L_{n}(q)(y)-q(y)\right|<\epsilon, \quad n \geq N(\epsilon), \quad y \in S^{m} .
$$

Daí, usando a condição $(i)$ obtemos

$$
\begin{aligned}
\left|L_{n}(f)(y)-f(y)\right| & \leq\left|L_{n}(f)(y)-L_{n}(q)(y)\right|+\left|L_{n}(q)(y)-q(y)\right|+|q(y)-f(y)| \\
& \leq\left|L_{n}(f-q)(y)\right|+\epsilon+\|q-f\|_{\infty} \\
& \leq\left\|L_{n}(f-q)\right\|_{\infty}+2 \epsilon \\
& =C\|(f-q)\|_{\infty}+2 \epsilon \\
& \leq(C+2) \epsilon, \quad n \geq N(\epsilon), \quad y \in S^{m}
\end{aligned}
$$

Portanto,

$$
\left\|L_{n}(f)-f\right\|_{\infty}<(C+2) \epsilon, \quad n \geq N(\epsilon) .
$$

Isto completa a prova do teorema. 


\section{Capítulo}

\section{Ordem de convergência do operador $T_{n}$}

Neste capítulo discutiremos ordens de convergência para $\left\|T_{n}(f)-f\right\|_{X}$, quando $n \rightarrow$ $\infty$, onde $T_{n}$ é o operador dado pela Fórmula (3.1). Utilizaremos o módulo de suavidade introduzido e discutido no Capítulo 2. Os resultados mais relevantes nesse sentido dependem de hipóteses de bi-zonalidade e da positividade sobre o núcleo $K_{n}$.

\subsection{A ordem de aproximação}

Antes de apresentarmos os resultados sobre ordem de convergência registraremos o lema abaixo, cuja prova segue da definição de $K_{n}$ e da ortonormalidade dos harmônicos esféricos.

Lema 4.1.1. Seja $K_{n}$ definido como no Teorema 3.1.1. Então

$$
\frac{1}{\sigma_{m}} \int_{S^{m}} K_{n}(x, y) d \sigma_{m}(y)=a_{01}(n), \quad x \in S^{m}, \quad n \in \mathbb{N} .
$$

Observamos que se $K_{n}(x, y) \geq 0, x, y \in S^{m}$, então segue do lema acima que $a_{01}(n) \geq 0$, $n \in \mathbb{N}$.

A análise da ordem de convergência será feita em duas estapas. Consideraremos primeiramente, o caso em que $X=C\left(S^{m}\right)$.

Teorema 4.1.2. Sejam $X=C\left(S^{m}\right), K_{n}$ um núcleo positivo e bi-zonal, e $\varphi: \mathbb{N} \rightarrow(0, \infty)$ uma função satisfazendo $\lim _{n \rightarrow \infty} \varphi(n)=0$. Se $a_{01}(n)=1, n \in \mathbb{N}, e$

$$
\left|1-a_{11}(n)\right|=O(\varphi(n)) \quad(n \rightarrow \infty)
$$

então existem um inteiro positivo $N_{0}$ e uma constante positiva $C$ tal que

$$
\left\|T_{n}(f)-f\right\|_{\infty} \leq C w_{1}(1-\varphi(n), f, X), \quad f \in C\left(S^{m}\right), \quad n \geq N_{0} .
$$

Demonstração: Seja $f \in C\left(S^{m}\right)$ e assuma que as seqüências $\left\{a_{k 1}(n)\right\}, k=0,1$ satisfazem as condições do enunciado. Como $K_{n}$ é bi-zonal, temos que $K_{n}(x, y)=L_{n}(\langle x, y\rangle), x, y \in S^{m}$, 
para alguma função $L_{n}$. Usando o Teorema 3.1.1 e o Lema 4.1.1, podemos escrever

$$
T_{n} f(x)-f(x)=\frac{1}{\sigma_{m}} \int_{S^{m}} L_{n}(\langle x, y\rangle) f(y) d \sigma_{m}(y)-\frac{f(x)}{\sigma_{m}} \int_{S^{m}} L_{n}(\langle x, y\rangle) d \sigma_{m}(y), \quad x \in S^{m} .
$$

Do Teorema 2.2.10 e da Fórmula de Funk-Hecke, segue que

$$
T_{n} f(x)-f(x)=\frac{\sigma_{m-1}}{\sigma_{m}} \int_{-1}^{1} L_{n}(t)\left[S_{t}^{m}(f)(x)-f(x)\right] d w_{m}(t), \quad x \in S^{m} .
$$

Logo, a não-negatividade de $L_{n}$ implica que

$$
\left|T_{n} f(x)-f(x)\right| \leq \frac{\sigma_{m-1}}{\sigma_{m}} \int_{-1}^{1} L_{n}(t)\left\|S_{t}^{m}(f)-f\right\|_{\infty} d w_{m}(t), \quad x \in S^{m},
$$

e portanto,

$$
\left\|T_{n}(f)-f\right\|_{\infty} \leq \frac{\sigma_{m-1}}{\sigma_{m}} \int_{-1}^{1} L_{n}(t)\left\|S_{t}^{m}(f)-f\right\|_{\infty} d w_{m}(t) .
$$

Da definição de módulo de suavidade, a desigualdade acima pode ser reduzida a

$$
\left\|T_{n}(f)-f\right\|_{\infty} \leq \frac{\sigma_{m-1}}{\sigma_{m}} \int_{-1}^{1} L_{n}(t) w_{1}(t, f, X) d w_{m}(t) .
$$

Como $\lim _{n \rightarrow \infty} \varphi(n)=0$, existe $N \in \mathbb{N}$ tal que $0<\varphi(n)<2, n \geq N$. Logo, o Corolário 2.9.3 garante que existe uma constante $C(1)$ tal que

$$
\begin{aligned}
\left\|T_{n}(f)-f\right\|_{\infty} \leq & C(1)\left(\frac{\sigma_{m-1}}{\sigma_{m}} \int_{-1}^{1} L_{n}(t)\left(1+\frac{1-t}{\varphi(n)}\right) d w_{m}(t)\right) w_{1}(1-\varphi(n), f, X) \\
= & C(1) \frac{\sigma_{m-1}}{\sigma_{m}}\left[\int_{-1}^{1} L_{n}(t) d w_{m}(t)+\frac{1}{\varphi(n)} \int_{-1}^{1} L_{n}(t) d w_{m}(t)\right. \\
& \left.\quad-\frac{1}{\varphi(n)} \int_{-1}^{1} t L_{n}(t) d w_{m}(t)\right] w_{1}(1-\varphi(n), f, X), \quad n \geq N .
\end{aligned}
$$

Mas, se $x_{0}$ é um ponto qualquer de $S^{m}$, usando novamente a Fórmula de Funk-Hecke e o Lema 4.1.1, obtemos

$$
\frac{\sigma_{m-1}}{\sigma_{m}} \int_{-1}^{1} L_{n}(t) d w_{m}(t)=\frac{1}{\sigma_{m}} \int_{S^{m}} L_{n}\left(\left\langle x_{0}, y\right\rangle\right) d \sigma_{m}(y)=1
$$

e

$$
\frac{\sigma_{m-1}}{\sigma_{m}} \int_{-1}^{1} t L_{n}(t) d w_{m}(t)=\frac{1}{\sigma_{m}} \int_{S^{m}} L_{n}\left(\left\langle x_{0}, y\right\rangle\right)\left\langle y, x_{0}\right\rangle d \sigma_{m}(y) .
$$

Observando que $\left\langle y, x_{0}\right\rangle=P_{1}^{m}\left(\left\langle y, x_{0}\right\rangle\right), y \in S^{m}$, e usando o Teorema da Adição, segue que

$$
\left\langle y, x_{0}\right\rangle=\frac{1}{N(m, 1)} \sum_{\nu=1}^{N(m, 1)} Y_{1 \nu}(y) \overline{Y_{1 \nu}\left(x_{0}\right)}
$$

Ainda, pela Proposição 3.1.2, podemos escrever

$$
L_{n}\left(\left\langle x_{0}, y\right\rangle\right)=\sum_{k=0}^{n} a_{k 1}(n) \sum_{l=1}^{N(m, k)} Y_{k l}\left(x_{0}\right) \overline{Y_{k l}(y)}
$$


Voltando em (4.2) e usando a ortonormalidade dos harmônicos esféricos, obtemos

$$
\begin{aligned}
\frac{1}{\sigma_{m}} \int_{S^{m}} L_{n}\left(\left\langle x_{0}, y\right\rangle\right)\left\langle y, x_{0}\right\rangle d \sigma_{m}(y) & =\frac{1}{N(m, 1)} a_{11}(n) \sum_{\nu=1}^{N(m, 1)} Y_{1 \nu}\left(x_{0}\right) \overline{Y_{1 \nu}\left(x_{0}\right)} d \sigma_{m}(y) \\
& =a_{11}(n) P_{1}^{m}\left(\left\langle x_{0}, x_{0}\right\rangle\right) \\
& =a_{11}(n), \quad n \geq 1 .
\end{aligned}
$$

Assim, tomando-se $N_{0} \in \mathbb{N}$ com $N_{0} \geq 1$ e $N_{0} \geq N$, obtemos

$$
\left\|T_{n}(f)-f\right\|_{\infty} \leq C(1)\left[1+\frac{1}{\varphi(n)}\left(1-a_{11}(n)\right)\right] w_{1}(1-\varphi(n), f, X), \quad n \geq N_{0} .
$$

Da hipótese (4.1), segue que existe uma constante positiva $C_{1}$ tal que

$$
\frac{1-a_{11}(n)}{\varphi(n)}<C_{1}, \quad n \in \mathbb{N}
$$

Logo,

$$
\left\|T_{n}(f)-f\right\|_{\infty} \leq C(1)\left(1+C_{1}\right) w_{1}(1-\varphi(n), f, X), \quad n \geq N_{0} .
$$

Portanto, existe uma constante positiva $C$ tal que

$$
\left\|T_{n}(f)-f\right\|_{\infty} \leq C w_{1}(1-\varphi(n), f, X), \quad n \geq N_{0}
$$

completando a prova do teorema.

Analisemos, agora, o caso $L^{p}\left(S^{m}\right), 1 \leq p<\infty$.

Teorema 4.1.3. Sejam $X=L^{p}\left(S^{m}\right), 1 \leq p<\infty$, $K_{n}$ um núcleo positivo e bi-zonal, e $\varphi: \mathbb{N} \rightarrow(0, \infty)$ a função definida no Teorema 4.1.2. Se $a_{01}(n)=1, n \in \mathbb{N}, e$

$$
\left|1-a_{11}(n)\right|=O(\varphi(n)) \quad(n \rightarrow \infty)
$$

então existem um inteiro positivo $N_{0}$ e uma constante positiva $C$ tal que

$$
\left\|T_{n}(f)-f\right\|_{p} \leq C w_{1}(1-\varphi(n), f, X), \quad f \in L^{p}\left(S^{m}\right), \quad n \geq N_{0} .
$$

Demonstração: Seja $f \in L^{p}\left(S^{m}\right), 1 \leq p<\infty$. Da prova do Teorema 4.1.2, temos que

$$
T_{n} f(x)-f(x)=\frac{\sigma_{m-1}}{\sigma_{m}} \int_{-1}^{1} L_{n}(t)\left[S_{t}^{m}(f)(x)-f(x)\right] d w_{m}(t), \quad x \in S^{m} .
$$

Logo, usando a desigualdade de Minkowski para integrais segue que

$$
\begin{aligned}
\left\|T_{n}(f)-f\right\|_{p} & =\frac{\sigma_{m-1}}{\sigma_{m}}\left\|\int_{-1}^{1} L_{n}(t)\left[S_{t}^{m}(f)(\cdot)-f(\cdot)\right] d w_{m}(t)\right\|_{p} \\
& \leq \frac{\sigma_{m-1}}{\sigma_{m}} \int_{-1}^{1} L_{n}(t)\left\|S_{t}^{m}(f)-f\right\|_{p} d w_{m}(t) .
\end{aligned}
$$

Portanto, a prova segue como a do Teorema 4.1.2. 
Corolário 4.1.4. Sejam $K_{n}$ um núcleo positivo e bi-zonal, e $\varphi: \mathbb{N} \rightarrow(0,2)$ uma função satisfazendo $\lim _{n \rightarrow \infty} \varphi(n)=0$. Se $a_{01}(n)=1, n \in \mathbb{N}, e$

$$
\left|1-a_{11}(n)\right|=O(\varphi(n)) \quad(n \rightarrow \infty)
$$

então existe uma constante positiva $C$ tal que

$$
\left\|T_{n}(f)-f\right\|_{X} \leq C w_{1}(1-\varphi(n), f, X), \quad f \in X, \quad n \in \mathbb{N} .
$$

Vejamos agora as recíprocas dos teoremas acima.

Teorema 4.1.5. Sejam $X=C\left(S^{m}\right)$, $K_{n}$ um núcleo positivo e bi-zonal, e $\varphi: \mathbb{N} \rightarrow(0, \infty)$ como no Teorema 4.1.2. Se existir um inteiro positivo $N_{1}$ e uma constante positiva $C$ tal que

$$
\left\|T_{n}(f)-f\right\|_{\infty} \leq C w_{1}(1-\varphi(n), f, X), \quad f \in C\left(S^{m}\right), \quad n \geq N_{1},
$$

então

$$
\left|1-a_{11}(n)\right|=O(\varphi(n)) \quad(n \rightarrow \infty)
$$

Demonstração: Usando o fato de $K_{n}$ ser bi-zonal e a ortonormalidade dos harmônicos esféricos, temos que

$$
\begin{aligned}
T_{n}\left(Y_{11}\right) & =\sum_{k=0}^{n} \sum_{l=1}^{N(m, k)} a_{k l}(n) \widehat{Y_{11}}(k, l) Y_{k l} \\
& =\sum_{k=0}^{n} a_{k 1}(n) \sum_{l=1}^{N(m, k)}\left(\frac{1}{\sigma_{m}} \int_{S^{m}} Y_{11}(y) \overline{Y_{k l}(y)} d \sigma_{m}(y)\right) Y_{k l} \\
& =a_{11}(n) Y_{11}, \quad n \geq 1 .
\end{aligned}
$$

Como $S^{m}$ é um conjunto compacto e $Y_{11}$ é uma função contínua, escolhemos $x_{0} \in S^{m}$ tal que $\left\|Y_{11}\right\|_{\infty}=\left|Y_{11}\left(x_{0}\right)\right|$. Como $Y_{11}\left(x_{0}\right) \neq 0$ podemos escrever

$$
a_{11}(n)=\frac{T_{n} Y_{11}\left(x_{0}\right)}{Y_{11}\left(x_{0}\right)}, \quad n \geq 1
$$

Logo,

$$
\begin{aligned}
\left|1-a_{11}(n)\right| & =\left|1-\frac{T_{n} Y_{11}\left(x_{0}\right)}{Y_{11}\left(x_{0}\right)}\right| \\
& =\left|\frac{Y_{11}\left(x_{0}\right)-T_{n} Y_{11}\left(x_{0}\right)}{Y_{11}\left(x_{0}\right)}\right| \\
& \leq \frac{\left\|Y_{11}-T_{n}\left(Y_{11}\right)\right\|_{\infty}}{\left\|Y_{11}\right\|_{\infty}}, \quad n \geq 1 .
\end{aligned}
$$

Seja $N_{0} \geq N_{1}$ tal que $0<\varphi(n)<2, n \geq N_{0}$. Pela Proposição 2.8.3, existe uma constante $M(1)$ tal que

$$
w_{1}\left(1-\varphi(n), Y_{11}, X\right) \leq M(1) \varphi(n)\left\|D^{1}\left(Y_{11}\right)\right\|_{\infty}, \quad n \geq N_{0}
$$


Por outro lado, a Proposição 2.3.2 revela que $D^{1}\left(Y_{11}\right)=Y_{11}$. Isso por sua vez, implica que $\left\|D^{1}\left(Y_{11}\right)\right\|_{\infty}=\left\|Y_{11}\right\|_{\infty}$. Logo, se (4.4) vale, obtemos

$$
\begin{aligned}
\left|1-a_{11}(n)\right| & \leq \frac{C}{\left\|Y_{11}\right\|_{\infty}} w_{1}\left(1-\varphi(n), Y_{11}, X\right) \\
& \leq \frac{C M(1)}{\left\|Y_{11}\right\|_{\infty}} \varphi(n)\left\|D^{1}\left(Y_{11}\right)\right\|_{\infty} \\
& =C M(1) \varphi(n), \quad n \geq N_{0} .
\end{aligned}
$$

O teorema segue.

Teorema 4.1.6. Sejam $X=L^{p}\left(S^{m}\right), 1 \leq p<\infty, K_{n}$ um núcleo positivo e bi-zonal, e $\varphi: \mathbb{N} \rightarrow(0, \infty)$ como no Teorema 4.1.2. Se existir um inteiro positivo $N_{1}$ e uma constante positiva $C$ tal que

$$
\left\|T_{n}(f)-f\right\|_{p} \leq C w_{1}(1-\varphi(n), f, X), \quad f \in L^{p}\left(S^{m}\right), \quad n \geq N_{1},
$$

então

$$
\left|1-a_{11}(n)\right|=O(\varphi(n)) \quad(n \rightarrow \infty) .
$$

Demonstração: Da prova do Teorema 4.1.5, segue que

$$
\left(a_{11}(n)-1\right) Y_{11}=T_{n}\left(Y_{11}\right)-Y_{11}, \quad n \geq 1
$$

Logo,

$$
\begin{aligned}
\left|a_{11}(n)-1\right|^{p}\left\|Y_{11}\right\|_{p}^{p} & =\frac{1}{\sigma_{m}} \int_{S^{m}}\left|Y_{11}(x)\right|^{p}\left|a_{11}(n)-1\right|^{p} d \sigma_{m}(x) \\
& =\frac{1}{\sigma_{m}} \int_{S^{m}}\left|T_{n} Y_{11}(x)-Y_{11}(x)\right|^{p} d \sigma_{m}(x) \\
& =\left\|T_{n}\left(Y_{11}\right)-Y_{11}\right\|_{p}^{p},
\end{aligned}
$$

ou seja,

$$
\left|a_{11}(n)-1\right|\left\|Y_{11}\right\|_{p}=\left\|T_{n}\left(Y_{11}\right)-Y_{11}\right\|_{p}, \quad n \geq 1 .
$$

Como $Y_{11} \not \equiv 0$, podemos escrever

$$
\left|a_{11}(n)-1\right|=\frac{\left\|T_{n}\left(Y_{11}\right)-Y_{11}\right\|_{p}}{\left\|Y_{11}\right\|_{p}}, \quad n \geq 1 .
$$

O restante da prova segue como a do Teorema 4.1.5.

Corolário 4.1.7. Sejam $K_{n}$ um núcleo positivo e bi-zonal, e $\varphi: \mathbb{N} \rightarrow(0, \infty)$ como no Teorema 4.1.2. Suponha que $a_{01}(n)=1, n \in \mathbb{N}$. Então as seguintes afirmações são equivalentes:

(i) $\left|1-a_{11}(n)\right|=O(\varphi(n))(n \rightarrow \infty)$;

(ii) Existem um inteiro positivo $N_{1}$ e uma constante positiva $C$ tal que

$$
\left\|T_{n}(f)-f\right\|_{X} \leq C w_{1}(1-\varphi(n), f, X), \quad f \in X, \quad n \geq N_{1} .
$$


Corolário 4.1.8. Sejam $K_{n}$ um núcleo positivo e bi-zonal, e $\varphi: \mathbb{N} \rightarrow(0,2)$ como no Teorema 4.1.2. Suponha que $a_{01}(n)=1, n \in \mathbb{N}$. Então as seguintes afirmações são equivalentes:

$(i)\left|1-a_{11}(n)\right|=O(\varphi(n))(n \rightarrow \infty)$;

(ii) Existe uma constante positiva $C$ tal que

$$
\left\|T_{n}(f)-f\right\|_{X} \leq C w_{1}(1-\varphi(n), f, X), \quad f \in X, \quad n \in \mathbb{N} .
$$

Observação 4.1.9. Se $a_{11}(n)<1, n \in \mathbb{N}$, e $\lim _{n \rightarrow \infty} a_{11}(n)=1$, uma escolha natural para a função $\varphi$ nos resultados acima é $\varphi(n)=1-a_{11}(n), n \in \mathbb{N}$.

Para provar os teoremas acima, consideramos a condição $a_{01}(n)=1, n \in \mathbb{N}$. Provaremos agora resultados semelhantes aos Teoremas 4.1.2 e 4.1.3, impondo uma condição mais fraca nos coeficientes $a_{01}(n)$, e uma outra condição envolvendo os coeficientes $a_{01}(n)$ e $a_{11}(n)$. A estimativa resultante depende agora da norma da função a ser aproximada.

Teorema 4.1.10. Sejam $X=C\left(S^{m}\right), K_{n}$ um núcleo positivo e bi-zonal, e $\varphi: \mathbb{N} \rightarrow(0, \infty)$ uma função como no Teorema 4.1.2. Se $\lim _{n \rightarrow \infty} a_{01}(n)=1 e$

$$
\left|1-a_{01}(n)\right|=O(\varphi(n))=\left|1-a_{11}(n)\right| \quad(n \rightarrow \infty)
$$

então existem $N_{0} \in \mathbb{N}$ e constantes positivas $C_{1}$ e $C_{2}$ tal que

$$
\left\|T_{n}(f)-f\right\|_{\infty} \leq C_{1} w_{1}(1-\varphi(n), f, X)+C_{2} \varphi(n)\|f\|_{\infty}, \quad n \geq N_{0}, \quad f \in C\left(S^{m}\right) .
$$

Demonstração: A prova é bem semelhante à do Teorema 4.1.2. Seja $f \in C\left(S^{m}\right)$ e escrevamos $K_{n}(x, y)=L_{n}(\langle x, y\rangle), x, y \in S^{m}$. Usando o Teorema 3.1.1 e o Lema 4.1.1, podemos escrever $\left(x \in S^{m}\right)$

$$
T_{n} f(x)-f(x)=\frac{1}{\sigma_{m}} \int_{S^{m}} L_{n}(\langle x, y\rangle) f(y) d \sigma_{m}(y)-\frac{f(x)}{\sigma_{m} a_{01}(n)} \int_{S^{m}} L_{n}(\langle x, y\rangle) d \sigma_{m}(y),
$$

contanto que $a_{01}(n) \neq 0$. Logo, do Teorema 2.2.10 e da Fórmula de Funk-Hecke, obtemos

$$
\begin{aligned}
T_{n} f(x)-f(x) & =\frac{\sigma_{m-1}}{\sigma_{m}} \int_{-1}^{1} L_{n}(t)\left(S_{t}^{m}(f)(x)-\frac{f(x)}{a_{01}(n)}\right) d w_{m}(t) \\
& =\frac{\sigma_{m-1}}{\sigma_{m} a_{01}(n)} \int_{-1}^{1} L_{n}(t)\left[a_{01}(n) S_{t}^{m}(f)(x)-f(x)\right] d w_{m}(t)
\end{aligned}
$$

Logo, usando o fato de que $a_{01}(n) \geq 0, n \in \mathbb{N}$, temos que

$$
\begin{aligned}
\left|T_{n} f(x)-f(x)\right| & \leq \frac{\sigma_{m-1}}{\sigma_{m} a_{01}(n)} \int_{-1}^{1} L_{n}(t)\left|a_{01}(n) S_{t}^{m}(f)(x)-f(x)\right| d w_{m}(t) \\
& \leq \frac{\sigma_{m-1}}{\sigma_{m} a_{01}(n)} \int_{-1}^{1} L_{n}(t)\left\|a_{01}(n) S_{t}^{m}(f)-f\right\|_{\infty} d w_{m}(t), \quad x \in S^{m}
\end{aligned}
$$


Como

$$
\left\|a_{01}(n) S_{t}^{m}(f)-f\right\|_{\infty} \leq\left|a_{01}(n)-1\right|\left\|S_{t}^{m}(f)\right\|_{\infty}+\left\|S_{t}^{m}(f)-f\right\|_{\infty}
$$

segue que

$$
\begin{aligned}
\left\|T_{n}(f)-f\right\|_{\infty} & \leq \frac{\sigma_{m-1}}{\sigma_{m} a_{01}(n)} \int_{-1}^{1} L_{n}(t)\left\|a_{01}(n) S_{t}^{m}(f)-f\right\|_{\infty} d w_{m}(t) \\
& \leq \frac{\sigma_{m-1}}{\sigma_{m} a_{01}(n)}\left[\left|a_{01}(n)-1\right| \int_{-1}^{1} L_{n}(t)\left\|S_{t}^{m}(f)\right\|_{\infty} d w_{m}(t)\right. \\
& \left.\quad+\int_{-1}^{1} L_{n}(t)\left\|S_{t}^{m}(f)-f\right\|_{\infty} d w_{m}(t)\right] .
\end{aligned}
$$

A Proposição 2.2.4-(i), a Fórmula de Funk-Hecke e o Lema 4.1.1, implicam que

$$
\begin{aligned}
\frac{\sigma_{m-1}}{\sigma_{m}} \int_{-1}^{1} L_{n}(t)\left\|S_{t}^{m}(f)\right\|_{\infty} d w_{m}(t) & \leq \frac{\sigma_{m-1}}{\sigma_{m}}\|f\|_{\infty} \int_{-1}^{1} L_{n}(t) d w_{m}(t) \\
& =\frac{\|f\|_{\infty}}{\sigma_{m}} \int_{S^{m}} L_{n}\left(\left\langle x_{0}, y\right\rangle\right) d \sigma_{m}(y) \\
& =\|f\|_{\infty} a_{01}(n),
\end{aligned}
$$

onde $x_{0} \in S^{m}$. Usando um procedimento similar ao do Teorema 4.1.2, podemos encontrar $N_{0} \in \mathbb{N}$ tal que

$$
\frac{\sigma_{m-1}}{\sigma_{m}} \int_{-1}^{1} L_{n}(t)\left\|S_{t}^{m}(f)-f\right\|_{\infty} d w_{m}(t) \leq\left[1+\frac{a_{01}(n)-a_{11}(n)}{\varphi(n)}\right] w_{1}(1-\varphi(n), f, X)
$$

e $a_{01}(n) \neq 0$, quando $n \geq N_{0}$. Se (4.6) vale, existe $C>0$ tal que

$$
\frac{a_{01}(n)-a_{11}(n)}{\varphi(n)} \leq C, \quad n \in \mathbb{N}
$$

Daí,

$$
\frac{\sigma_{m-1}}{\sigma_{m}} \int_{-1}^{1} L_{n}(t)\left\|S_{t}^{m}(f)-f\right\|_{\infty} d w_{m}(t) \leq(1+C) w_{1}(1-\varphi(n), f, X), \quad n \geq N_{0} .
$$

Juntando-se essas informações, vem que

$$
\left\|T_{n}(f)-f\right\|_{\infty} \leq\left|a_{01}(n)-1\right|\|f\|_{\infty}+\frac{1+C}{a_{01}(n)} w_{1}(1-\varphi(n), f, X), \quad n \geq N_{0} .
$$

Se $\lim _{n \rightarrow \infty} a_{01}(n)=1$, existe uma constante positiva $C_{1}$ tal que

$$
\frac{1+C}{a_{01}(n)} \leq C_{1}, \quad n \in \mathbb{N}
$$

enquanto que (4.6) garante a existência de $C_{2}>0$ tal que

$$
\left|a_{01}(n)-1\right|<C_{2} \varphi(n), \quad n \in \mathbb{N} .
$$

Portanto,

$$
\left\|T_{n}(f)-f\right\|_{\infty} \leq C_{1} w_{1}(1-\varphi(n), f, X)+C_{2} \varphi(n)\|f\|_{\infty}, \quad n \geq N_{0} .
$$

Isso completa a prova do teorema. 
Teorema 4.1.11. Sejam $X=L^{p}\left(S^{m}\right), 1 \leq p<\infty, K_{n}$ um núcleo positivo e bi-zonal e $\varphi: \mathbb{N} \rightarrow(0, \infty)$ como no Teorema 4.1.2. Se $\lim _{n \rightarrow \infty} a_{01}(n)=1 e$

$$
\left|1-a_{01}(n)\right|=O(\varphi(n))=\left|1-a_{11}(n)\right| \quad(n \rightarrow \infty)
$$

então existem $N_{0} \in \mathbb{N}$ e constantes positivas $C_{1}$ e $C_{2}$ tal que

$$
\left\|T_{n}(f)-f\right\|_{p} \leq C_{1} w_{1}(1-\varphi(n), f, X)+C_{2} \varphi(n)\|f\|_{p}, \quad n \geq N_{0}, \quad f \in L^{p}\left(S^{m}\right) .
$$

Demonstração: Seja $f \in L^{p}\left(S^{m}\right)$. Da prova do teorema anterior, sabemos que

$$
T_{n} f(x)-f(x)=\frac{\sigma_{m-1}}{\sigma_{m} a_{01}(n)} \int_{-1}^{1} L_{n}(t)\left[a_{01}(n) S_{t}^{m}(f)(x)-f(x)\right] d w_{m}(t), \quad x \in S^{m} .
$$

A desigualdade de Minkowski para integrais implica que

$$
\left\|T_{n}(f)-f\right\|_{p} \leq \frac{\sigma_{m-1}}{\sigma_{m} a_{01}(n)} \int_{-1}^{1} L_{n}(t)\left\|a_{01}(n) S_{t}^{m}(f)-f\right\|_{p} d w_{m}(t) .
$$

O restante da prova segue como a do teorema anterior.

Exigindo-se que $a_{01}(n) \neq 0, n \in \mathbb{N}$, e que a função $\varphi(n) \in(0,2), n \in \mathbb{N}$, obtemos o seguinte resultado.

Teorema 4.1.12. Sejam $K_{n}$ um núcleo positivo e bi-zonal e $\varphi: \mathbb{N} \rightarrow(0,2)$ como no Teorema 4.1.2. Se $a_{01}(n) \neq 0, n \in \mathbb{N}, \lim _{n \rightarrow \infty} a_{01}(n)=1 e$

$$
\left|1-a_{01}(n)\right|=O(\varphi(n))=\left|1-a_{11}(n)\right| \quad(n \rightarrow \infty)
$$

então constantes positivas $C_{1}$ e $C_{2}$ tal que

$$
\left\|T_{n}(f)-f\right\|_{X} \leq C_{1} w_{1}(1-\varphi(n), f, X)+C_{2} \varphi(n)\|f\|_{X}, \quad n \in \mathbb{N}, \quad f \in X .
$$

O resultado abaixo é conseqüência do Corolário 3.3.4 e dos Teoremas 4.1.10 e 4.1.11. Ele coloca as estimativas obtidas no contexto do Capítulo 3.

Corolário 4.1.13. Sejam $K_{n}$ um núcleo positivo e bi-zonal, e $\varphi: \mathbb{N} \rightarrow(0, \infty)$ como no Teorema 4.1.2. Se $\left\{T_{n}\right\}_{n \in \mathbb{N}}$ é aproximação da identidade em $X$ e

$$
\left|1-a_{01}(n)\right|=O(\varphi(n))=\left|1-a_{11}(n)\right| \quad(n \rightarrow \infty)
$$

então existem $N_{0} \in \mathbb{N}$ e constantes positivas $C_{1}$ e $C_{2}$ tal que

$$
\left\|T_{n}(f)-f\right\|_{X} \leq C_{1} w_{1}(1-\varphi(n), f, X)+C_{2} \varphi(n)\|f\|_{X}, \quad n \geq N_{0}, \quad f \in X .
$$




\section{Problemas abertos}

Comentaremos aqui sobre algumas questões relativas ao trabalho, umas que não conseguimos resolver, outras que nem chegamos a pensar. De qualquer forma, pretendemos considerá-las como propostas de trabalho para o futuro.

No Capítulo 3, calculamos a norma do operador de aproximação $T_{n}$ nos casos $X=C\left(S^{m}\right)$ e $X=L^{1}\left(S^{m}\right)$. No caso $X=L^{p}\left(S^{m}\right), 1<p<\infty$, obtivemos apenas uma estimativa para sua norma. Parece-nos razoável esperar que o valor da norma neste caso restante ainda tenha a mesma expressão encontrada nos outros casos.

Quanto aos resultados envolvendo o módulo de suavidade, duas questões permanecem: analisar a possibilidade de obter-se resultados similares aos aqui obtidos, com hipóteses sobre os núcleos diferentes daquelas consideradas. Ainda, obter resultados mais refinados que envolvam os módulos de suavidade de ordem maior do que 1 ou mesmo outros módulos de suavidade. 


\section{Referências Bibliográficas}

[1] Askey, R., Orthogonal polynomials and special functions. Society for Industrial and Applied Mathematics, Philadelphia, Pa., 1975.

[2] Axler, S.; Bourdon, P.; Ramey, W., Harmonic function theory. Graduate Texts in Mathematics, 137. Springer-Verlag, New York, 1992.

[3] Berens, H.; Butzer, P. L.; Pawelke, S., Limitierungsverfahren von Reihen mehrdimensionaler Kugelfunktionen und deren Saturationsverhalten. (German) Publ. Res. Inst. Math. Sci. Ser. A 4 1968/1969, 201-268.

[4] Berens, H.; Li, L. Q., On the de la Vallée-Poussin means on the sphere. Results Math. 24 (1993), no. 1-2, 12-26.

[5] Brezis, H., Analyse fonctionnelle.(French) [Functional analysis] Théorie et applications [Theory and applications]. Collection Mathématiques Appliquées pour la Maitrise. [Collection of Applied Mathematics for the Master's Degree] Masson, Paris, 1983.

[6] Carlitz, L., The product of two ultraspherical polinomials, Proc. Glasgow Math. Assoc., 5 (1961), p. 76-79.

[7] Davis, P. J., Interpolation and approximation. Republication, with minor corrections, of the 1963 original, with a new preface and bibliography. Dover Publications, Inc., New York, 1975.

[8] DeVore, Ronald A.; Lorentz, George G., Constructive approximation. Grundlehren der Mathematischen Wissenschaften [Fundamental Principles of Mathematical Sciences], 303. Springer-Verlag, Berlin, 1993.

[9] Dougall, J., The product of two Legendre polynomials, Proc. Glasgow Math. Assoc., 1 (1953), 121-125.

[10] Folland, G. B., Real analysis. Modern techniques and their applications. Second edition. Pure and Applied Mathematics (New York). A Wiley-Interscience Publication. John Wiley \& Sons, Inc., New York, 1999. 
[11] Groemer, H., Geometric applications of Fourier series and spherical harmonics. Encyclopedia of Mathematics and its Applications, 61. Cambridge University Press, 1996.

[12] Horn, R. A.,; Johnson, C. R., Matrix analysis. Cambridge University Press, New York, 1990.

[13] Hylleraas, E., Linearization of products of Jacobi polinomials, Math. Scand., 10 (1962), 189-200.

[14] Körner, T. W., Fourier analysis. Cambridge University Press, Cambridge, 1988.

[15] Lasser, R.; Obermaier, J., On the convergence of weighted Fourier expansions with respect to orthogonal polynomials. Acta Sci. Math. (Szeged) 61 (1995), no. 1-4, 345-355.

[16] Lasser, R.; Obermaier, J., Orthogonal expansions for $L^{p}$ - and $C$-spaces. Special functions (Hong Kong, 1999), 194-206, World Sci. Publ., River Edge, NJ, 2000.

[17] Lizorkin, P. I.; Nikol'skiū, S. M., Approximation by spherical functions. (Russian) Studies in the theory of differentiable functions of several variables and its applications, 11 (Russian). Trudy Mat. Inst. Steklov. 173 (1986), 181-189.

[18] Lizorkin, P. I.; Nikol'skiŭ, S. M., Approximation theory on the sphere, Proc. Steklov Inst. Math., 172 (1987), 295-302.

[19] Menegatto, V. A., Approximation by spherical convolution, Numer. Funct. Anal. Optim. 18 (1997), no. 9-10, 995-1012.

[20] Menegatto, V. A.; Piantella, A. C. Approximation on the sphere by weighted Fourier expansions. J. Appl. Math. 2005, no. 4, 321-339.

[21] Menegatto, V. A.; Piantella, A. C. Old and new on the Laplace-Beltrami derivative: weighted approximation on the sphere. Submetido para publicação.

[22] Morimoto, M., Analytic functionals on the sphere. Translations of Mathematical Monographs, 178. American Mathematical Society, Providence, R.I., 1998.

[23] Müller, C., Analysis of spherical symmetries in euclidean spaces. Applied Mathematical Sciences, Vol. 129, Springer-Verlag, New York, 1998.

[24] Müller, C., Spherical harmonics. Lecture Notes in Mathematics, Vol. 17, Springer-Verlag, Berlin-New York, 1966.

[25] Munkres, J. R., Topology, a first course, Prentice-Hall,Inc., Englewood Cliffs, N.J., 1975. 
[26] Pawelke, S., Über die Approximationsordnung bei Kugelfunktionen und algebraischen Polynomen. (German) Tôhoku Math. J. (2) 24 (1972), 473-486.

[27] Reimer, M., Multivariate polynomial approximation. International Series of Numerical Mathematics, 144. Birkhäuser Verlag, Basel, 2003.

[28] Rudin, W., Uniqueness theory for Laplace series. Trans. Amer. Math. Soc. 68, (1950). 287-303.

[29] Rudin, W., Principles of mathematical analysis. Third edition. International Series in Pure and Applied Mathematics. McGraw-Hill Book Co., New York-Auckland-Düsseldorf, 1976.

[30] Schoenberg, I. J., Positive definite functions on sphere, Duke Math. J., 9 (1942), 96-108.

[31] Seeley, R. T., Spherical harmonics, American Math. Monthly, 73 (1966) no. 4 , Part II, 115-121.

[32] Shapiro, Harold S., Smoothing and approximation of functions. Revised and expanded edition of mimiographed notes (Matscience Report No. 55). Van Nostrand Reinhold Mathematical Studies. Van Nostrand Reinhold Co., New York-Toronto, Ontário-London, 1969.

[33] Stromberg, K.; Hewitt, E., Real and abstract analysis. A modern treatment of the theory of functions of a real variable. Second printing corrected. Springer-Verlag, New YorkBerlin, 1969.

[34] Szegö, G., Orthogonal polynomials, American Mathematical Society Colloquium Publications, v. 23. American Mathematical Society, New York, 1939.

[35] Kunyang, W.; Luoqing, L., Harmonic analysis and approximation on the unit sphere, Science Press, Beijing, 2000.

[36] Wehrens, M., Best approximation on the unit sphere in $R^{k}$, Functional analysis and approximation (Oberwolfach, 1980), pp. 233-245, Internat. Ser. Numer. Math., 60, Birkhäuser, Basel-Boston, Mass., 1981.

[37] Wehrens, M., Legendre-Transformationsmethoden und approximation von funktionen auf der einheitskugel in $R^{3}$. Doctoral Dissertation, RWTH Aachen, 1980. 


\section{Tabela de símbolos}

$S^{m}$
$\mathbb{S}_{x}^{m, t}$
$a_{k}^{m}(K)$
$\sigma_{m}$
$\sigma_{m}(A)$
$d \sigma_{m}$
$d y$
$d w_{m}$
$d(k, i, j)$
$C_{n}^{(m-1) / 2}$
$C\left(S^{m}\right)$
$D^{r}$
$\mathcal{H}_{n}\left(\mathbb{R}^{m+1}\right)$
$\mathcal{H}_{n}\left(S^{m}\right)$
$J^{r}$
$L^{p}\left(S^{m}\right)$
$L^{1, m}\left([-1,1], d w_{m}\right)$
$N(m, n)$
$\mathcal{P}_{n}\left(\mathbb{R}^{m+1}\right)$
$\mathcal{P}_{n}\left(S^{m}\right)$
$\mathbb{P}_{n}\left(S^{m}\right)$
$P_{n}^{m}$
$S_{t}^{m}$
$T_{n}$
$W_{X}^{r}$
$\mathcal{Y}_{n}$

${ }^{m}$

Esfera unitária em $\mathbb{R}^{m+1}$

Subesfera de $S^{m}$ com pólo $x$ e raio $\left(1-t^{2}\right)^{1 / 2}$

Coeficiente da Fórmula de Funk-Hecke

Área da superfície de $S^{m}$

Medida do conjunto $A$

Elemento de medida usual sobre $S^{m}$

Elemento de medida de $\mathbb{S}_{x}^{m, t}$

Elemento de medida sobre $[-1,1]$

Coeficiente da fórmula de linearização de Dougall

Polinômio de Gegenbauer de grau $n$ associado a $(m-1) / 2$

Espaço das funções contínuas definidas em $S^{m}$

Derivada forte de Laplace-Beltrami de ordem $r$

Espaço dos polinômios em $m+1$ variáveis que são harmônicos e homogêneos de grau $n$

Espaço dos harmônicos esféricos de grau $n$ em $m+1$ variáveis

Operador integral de Laplace-Beltrami de ordem $r$

Espaço das funções $f: S^{m} \rightarrow \mathbb{C}$ tal que $|f|^{p}$ é integrável em relação a $d \sigma_{m}$

Espaço das funções integráveis em relação a $d w_{m}$ sobre $[-1,1]$

Dimensão de $\mathcal{H}_{n}\left(S^{m}\right)$

Espaço dos polinômios em $m+1$ variáveis que são homogêneos de grau $n$

Espaço das restrições a $S^{m}$ dos polinômios homogêneos de grau $n$ em $m+1$ variáveis

Espaço dos polinômios esféricos de grau no máximo $n$ em $m+1$ variáveis

Polinômio de Legendre de grau $n$ associado à dimensão $m+1$

Operador translação esférica

Operador de aproximação

Espaço das funções diferenciáveis no sentido da derivada forte de Laplace-Beltrami de ordem $r$

Operador projeção esférica 
$\delta_{n k} \quad$ Função delta de Kronecker

$\Gamma \quad$ Função gama

$\Delta_{t} \quad$ Operador diferença esférica

$\Delta_{t}^{r} \quad r$-ésima diferença esférica

$\langle\cdot, \cdot\rangle \quad$ Produto interno usual de $\mathbb{R}^{m+1}$

$\langle\cdot, \cdot\rangle_{2} \quad$ Produto interno em $L^{2}\left(S^{m}\right)$ 


\section{Îndice Remissivo}

Aproximação da identidade, 64

em um ponto, 75

Coeficiente de Fourier, 9

Coeficiente de Fourier-Legendre , 20

Convolução esférica, 13, 26

Derivada forte de Laplace-Beltrami, 27

Desigualdade

de Minkowski para integrais, 23

Dimensão de $\mathcal{H}_{n}\left(S^{m}\right), 8$

Elemento de medida sobre $S^{m}, 5$

Espaço

$\mathbb{P}_{n}\left(S^{m}\right), 6$

$C\left(S^{m}\right), 5$

$L^{p}\left(S^{m}\right), 5$

$L^{1, m}\left([-1,1], d w_{m}\right), 12$

$W_{X}^{r}, 27$

$\mathcal{H}_{n}\left(S^{m}\right), 6$

$\mathcal{P}_{n}\left(S^{m}\right), 6$

Fórmula de Funk-Hecke, 12

Fórmula de linearização de Dougall, 14 Função

$G_{h}, 39$

$\mathcal{L}, 33$

$\mathcal{L}_{h}, 37$

$\mathfrak{L}, 31$

harmônica, 6

homogênea, 6

positiva definida, 13
Harmônicos esféricos, 6

Integral de Laplace-Beltrami, 31, 34

K-funcional, 55

Módulo de suavidade esférico, 51

Núcleo bi-zonal, 60

Norma

de $S_{t}^{m}, 22$

de $T_{n}, 61,63$

em $L^{p}\left(S^{m}\right), 5$

em $W_{X}^{r}, 29$

uniforme, 5

Operador

$T_{n}, 59$

auxiliar $A_{h}^{r}, 41$

diferença esférica, 25

integral de Laplace-Beltrami, 31, 34

projeção esférica, 17

Ordem de aproximação, 83

Polinômio

de Gegenbauer, 11

de Legendre, 11

esférico, 6

Problemas abertos, 91

Produto interno de $L^{2}\left(S^{m}\right), 6$

Teorema da Adição, 11

Teorema do produto de Schur, 14

Translação esférica, 21 